\title{
Laser Induced Fluorescence Studies of Electrostatic Double Layers in an Expanding Helicon Plasma
}

Jerry Carr Jr.

West Virginia University

Follow this and additional works at: https://researchrepository.wvu.edu/etd

\section{Recommended Citation}

Jerry Carr Jr., "Laser Induced Fluorescence Studies of Electrostatic Double Layers in an Expanding Helicon Plasma" (2013). Graduate Theses, Dissertations, and Problem Reports. 3648.

https://researchrepository.wvu.edu/etd/3648

This Dissertation is protected by copyright and/or related rights. It has been brought to you by the The Research Repository @ WVU with permission from the rights-holder(s). You are free to use this Dissertation in any way that is permitted by the copyright and related rights legislation that applies to your use. For other uses you must obtain permission from the rights-holder(s) directly, unless additional rights are indicated by a Creative Commons license in the record and/ or on the work itself. This Dissertation has been accepted for inclusion in WVU Graduate Theses, Dissertations, and Problem Reports collection by an authorized administrator of The Research Repository @ WVU.

For more information, please contact researchrepository@mail.wvu.edu. 


\title{
Laser Induced Fluorescence Studies of Electrostatic Double Layers in an Expanding Helicon Plasma
}

\author{
Jerry Carr Jr. \\ Dissertation submitted to the College of Arts and Sciences \\ at West Virginia University \\ in partial fulfillment of the requirements \\ for the degree of \\ Doctor of Philosophy \\ in \\ Physics \\ Earl Scime, PhD., Chair \\ Paul Cassak, PhD. \\ Fred L. King, PhD. \\ Amy M. Keesee, PhD. \\ Duncan Lorimer, $\mathrm{PhD}$. \\ Department of Physics \\ Morgantown, West Virginia \\ 2013
}

Keywords: helicon source, laser-induced fluorescence, double layer, wavelet time frequency analysis

Copyright 2013 Jerry Carr Jr. 


\section{Abstract}

\section{Laser Induced Fluorescence Studies of Electrostatic Double Layers in Expanding Helicon}

\section{Plasma}

Jerry Carr Jr.

We report the first evidence of a laboratory double layer (DL) collapsing in the presence of an instability studied by Chakraborty Thakur et al. ${ }^{1}$ with the use of time resolved laser induced fluorescence (LIF) studies. Higher time resolution studies then provided the first statistically validated proof of the correlation between the ion acoustic instability and a DL. Time-frequency analysis in the form of time resolved cross power spectra and continuous wavelet transforms were used to provide insight into beam formation. The implications of this work is that in the creation of strong DLs in expanding plasmas for plasma propulsion or other applications may be self-limited through instability growth.

Over the past decade, experimental and theoretical studies have demonstrated the formation of stable, electrostatic, current-free double layers (CFDLs) in plasmas with a strong density gradient; typically a result of a divergent magnetic field. In this work, we present evidence for the formation of multiple double layers within a single divergent magnetic field structure. Downstream of the divergent magnetic field, multiple accelerated ion populations are observed through laser induced fluorescence measurements of the ion velocity distribution function. The formation of the multiple double layer structure is a strong function of the neutral gas pressure in the experiment. The similarity of the accelerated ion populations observed in these laboratory experiments to ion populations observed in reconnection outflow regions in the magnetosphere and in numerical simulations is also described. If ion energization during magnetic reconnection also results solely from acceleration in electric fields, these observations imply a prediction that the ion heating, i.e., the broadening of ion velocity distribution functions, reported in magnetic reconnection experiments is more accurately described by a superposition of differently accelerated ion populations. Therefore, the ion "heating" rate during reconnection should scale as the square root of the cube of the charge per unit mass $\left(q^{3} / \mathrm{m}\right)^{1 / 2}$ for ions with varying charge-to-mass ratios.

A new RFEA probe was benchmarked on the low pressure CFDL plasmas produced in WVU HELIX-LEIA. This work was the result of collaboration between the University of Tromsø (UiT) and WVU. LIF was used to confirm the RFEAs ability to detect a beam when one was present. The RFEA was also able to detect the presence of a beam when LIF techniques were limited by metastable quenching. The probe's limitations in dealing with ion focusing are discussed as well.

${ }^{1}$ S. Chakraborty Thakur, Z. Harvey, I. A. Biloiu, A. Hansen, R. A. Hardin, W. S. Przybysz, and

E. E. Scime, Phys. Rev. Lett., 102, 035004 (2009). 


\section{Acknowledgements}

I could not have done this without the support of so many. I want to thank Dr. Earl Scime for the training, incredible career opportunities, and providing me the space to grow as a leader. Thank you to the rest of the physics department, especially Dr. Paul Cassak, Dr. Amy Keesee, Dr. Duncan Lorimer, Greg Puskar and Dr. Clayton Simien (along with his wife Dr. Daneesh Simien), all who have had a tremendous impact on my career. Thank you to the fantastic Physics Department support staff past and present, especially, Sherry Puskar, Valerie Burgess, Devon Cleland, Carl Weber, Doug Mathess and J.R. Raber.

I formed some wonderful relationships with members of the WVU physics community, specifically with Lucas Shepherd, Dr. Mitchell Mickaliger, Spencer Wolfe, Dustin McCarren, Dr. Matthew Galante, office mate Stephanie Sears, Colin Komar, Justin Elfritz, Mike Lindon and Njål Gulbrandsen. I greatly appreciate the training I received from Dr. Saikat Chakraborty Thakur, Dr. Robert Hardin, Dr. Saeid Houshmandyar, Dr. Alexander Hansen, and Dr. Richard Magee. I have been fortunate to work with an incredible pair of undergraduates, Greg Lusk and Robert Vandervort.

I want to recognize the following people outside of the physics department who have offered me encouragement and support: Dr. Krystal Frazier, Dr. Constinia Charbonnette, Dr. Christina Wilson, Dr. Tamara Lyn, Susan Johnson, John Gaddis, Wanda and Otis Cox, Dotty and Tom Wilson, and Professors Patricia and Daryl Lee. I have received powerful assistance from David Fryson, Sharon Mallow, Jennifer McIntosh, and Dr. Fred King.

The Georgia Tech cooperative program that placed me into the physics world with a position at ORNL was nothing short of phenomenal. Thank you to my fellow Yellow Jackets for 
reaching back and giving me exposure to large scale physics research: Thomas Mann Jr. and Dr. Robert Welton. Thanks to the many others who made my experience at the ORNL/SNS what propelled me into graduate school especially Dr. Martin Stockli, Sydney Murray II, Terry Pennisi, Dr. Richard Goulding, Justin Carmichael and Ted Williams.

I want to thank my father Jerry Carr Sr. for keeping my brother and I safe in the city of Detroit and making our education a top priority. To my mother Wanda Carey, I am so grateful for your love and support throughout my educational journey. You have been behind me always. Thank you to the rest of my family, including my brother Jason Carr and his bride to be, Mariel, my grandmothers, Shirley Pulley and Daisy Knott (along with Clarence), all of my aunts, uncles and cousins. I have received tremendous inspiration from all of you. Thank you to my wife's family, especially my mother-in-law Beryl Wilson, for keeping us near to your heart and for all of the support. To my wife Nicole, thank you for your love and strength. You are simply amazing.

This work was supported by NSF award PHY-0611571. I was also supported by the WV Space Grant, the SREB graduate fellowship and the Chancellor's Scholars Program. We acknowledge NASA contract NAS5-02099 and V. Angelopoulos for use of data from the THEMIS Mission, specifically C. W. Carlson and J. P. McFadden for use of ESA data. 


\section{Dedication}

To my wife, Nicole and our future together.

In Memory of My Grandfathers

Jeremiah Carr

Reginald Carey

Abram Cherry

William Pulley 


\section{Contents}

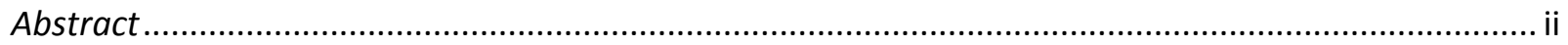

Acknowledgements ....................................................................................................

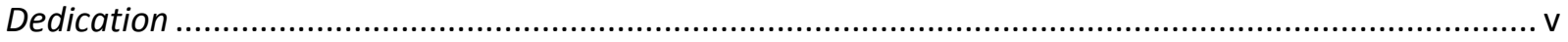

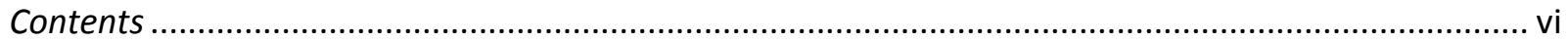

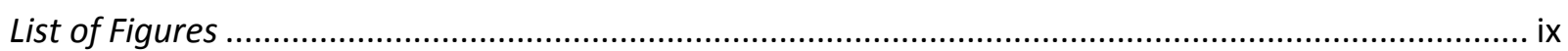

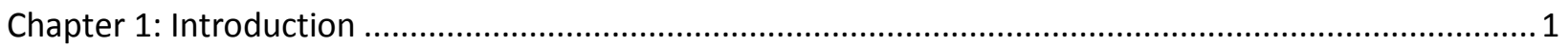

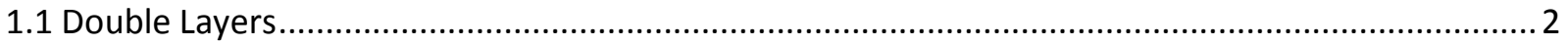

1.2 Space Observations of Current Free Double layers ........................................................... 5

1.3 Laboratory Studies of Double Layers ......................................................................... 9

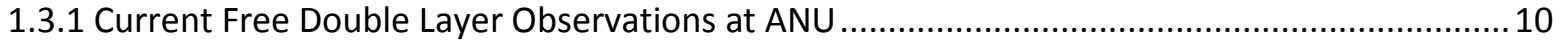

1.3.2 CFDLs in Expanding Helicon Plasmas Theory ..........................................................

1.3.3 Double Layer Laboratory Studies at WVU .............................................................. 16

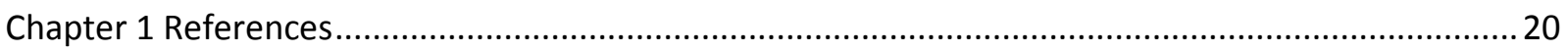

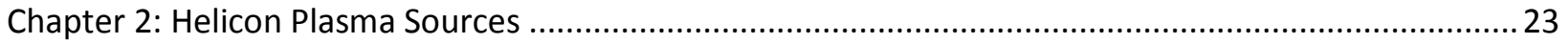

2.1 Introduction to Helicon Plasma Sources ......................................................................... 23

2.2 Physics of Helicon Plasma Sources........................................................................... 24

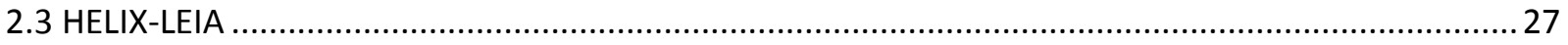

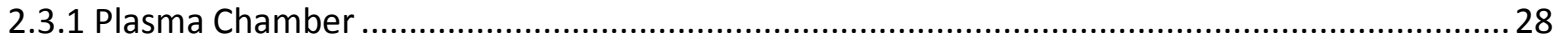

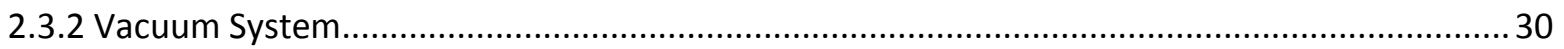

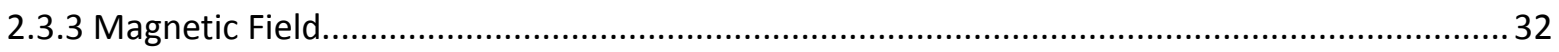

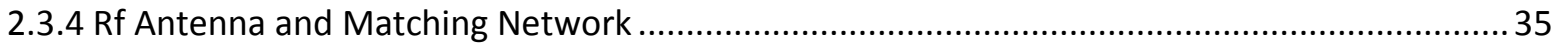

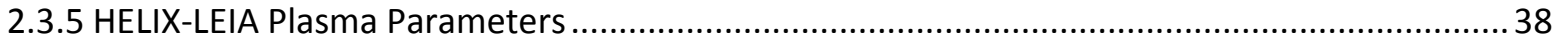

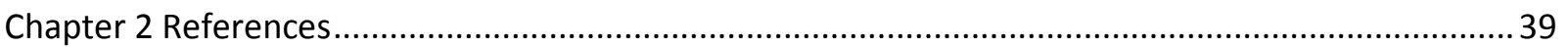

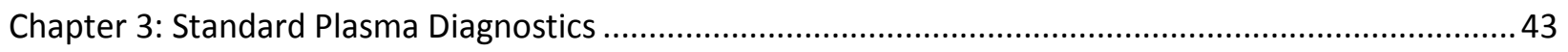

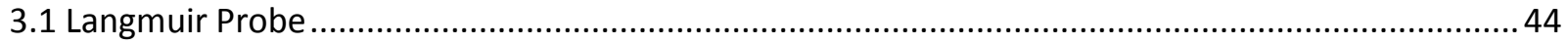

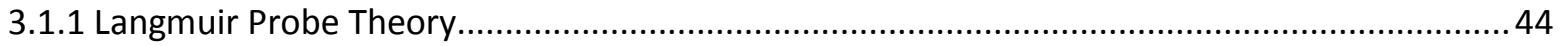

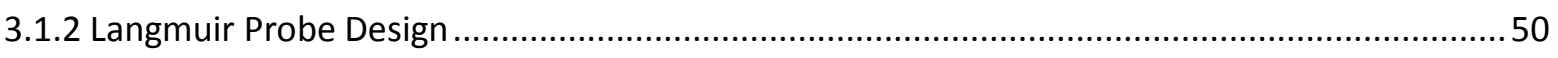

3.2 Electrostatic Probe ................................................................................................ 53

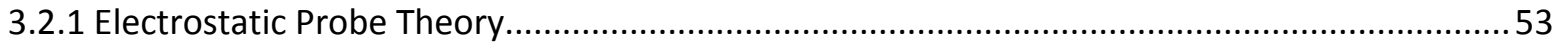




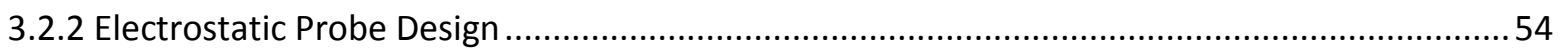

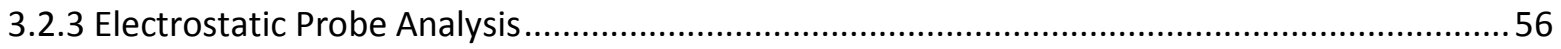

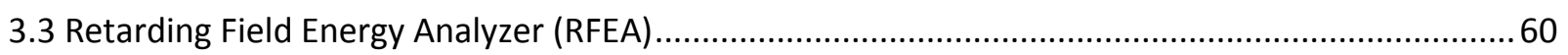

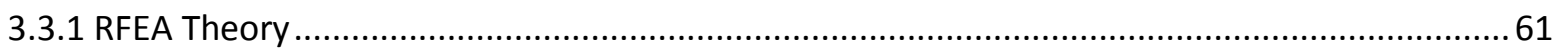

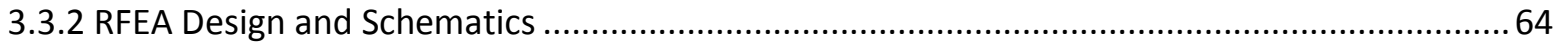

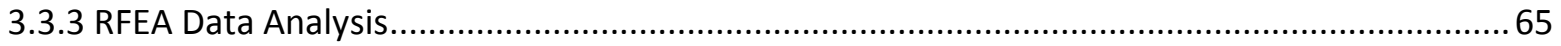

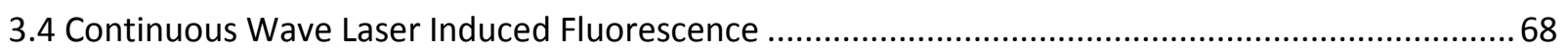

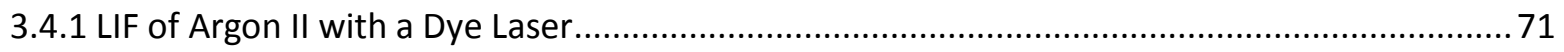

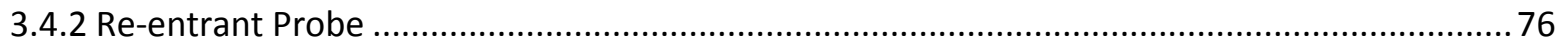

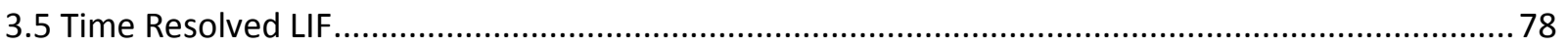

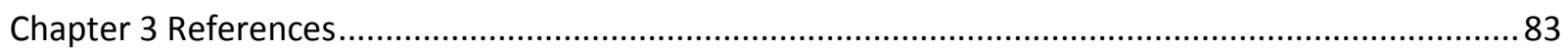

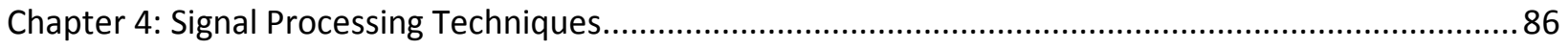

4.1 The Ion Acoustic Instability and Double Layer Formation ........................................................... 87

4.2 Fourier and Wavelet Transform Methods for Signal Processing ................................................ 92

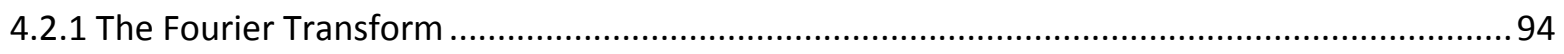

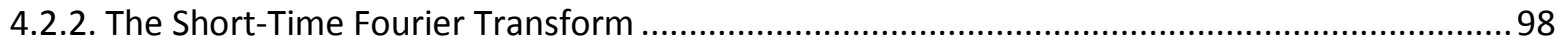

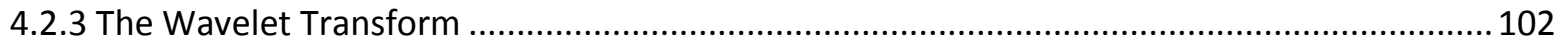

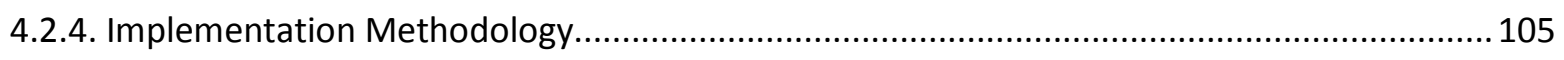

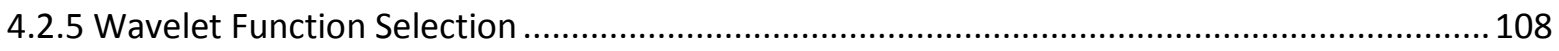

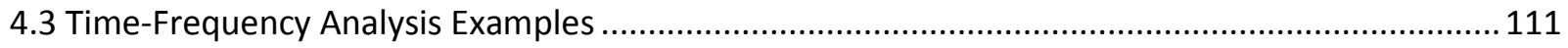

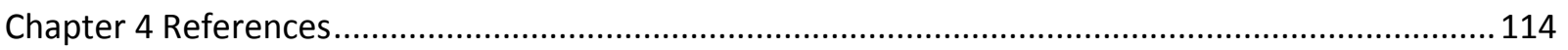

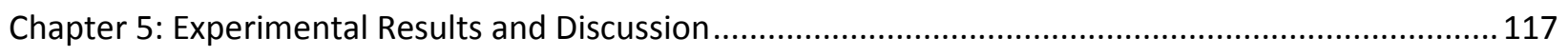

5.1 Time Resolved LIF Double Layer Studies ............................................................................ 117

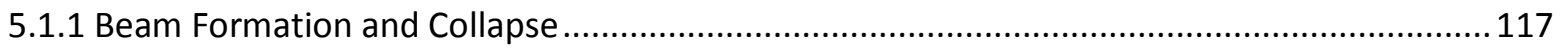

5.1.2 Analysis of Time Resolved Double Layer Study ............................................................. 123

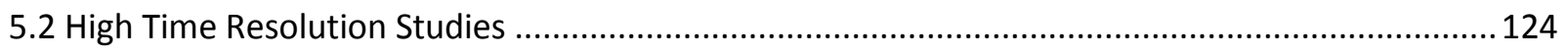

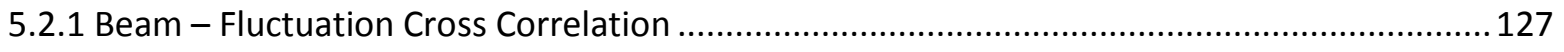

5.2.2 Wavelet-Based Fluctuation Analysis .................................................................................. 132

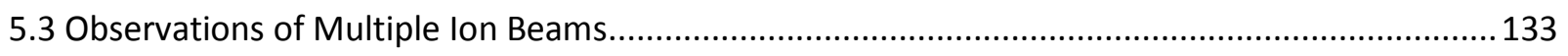

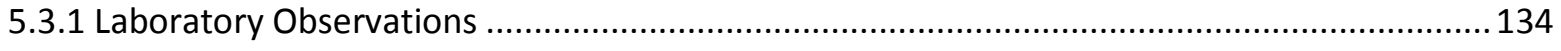

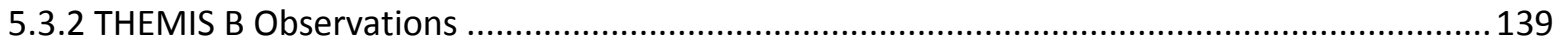

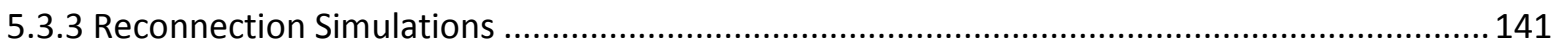


5.3.4 Implications of Multi-Beam Results for Reconnection Driven Ion Heating Studies ..... 142

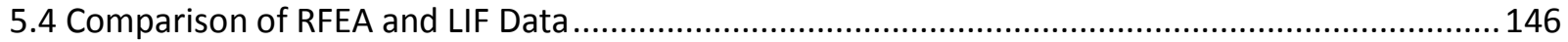

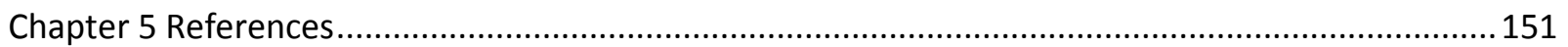

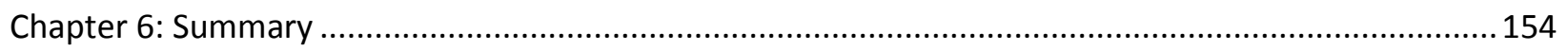

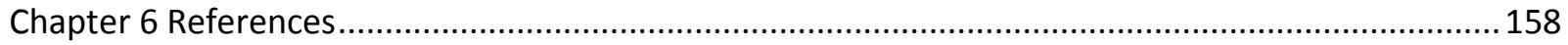




\section{List of Figures}

Chapter 1: Introduction

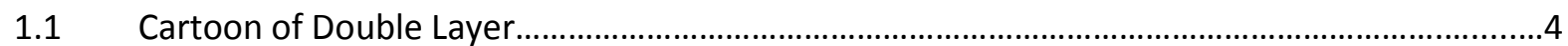

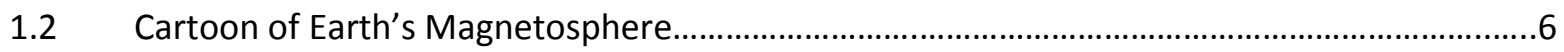

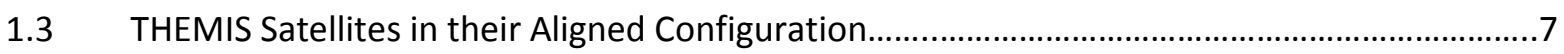

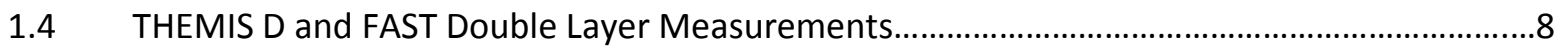

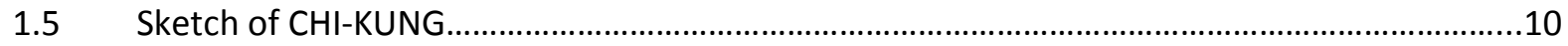

1.6 Schematic Interpreting the Observations from FAST and CHI-KUNG...................................12

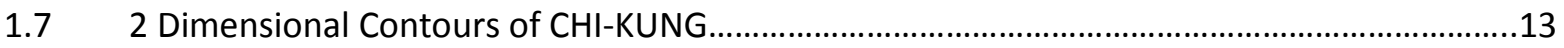

$1.8 \quad$ Ion Beam Velocity versus Antenna Frequency........................................................................17

Chapter 2 Helicon Plasma Sources

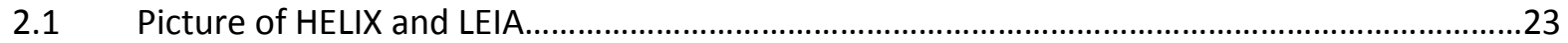

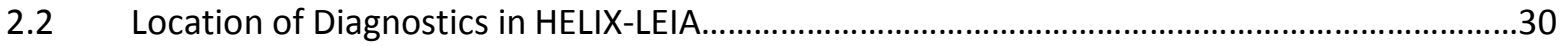

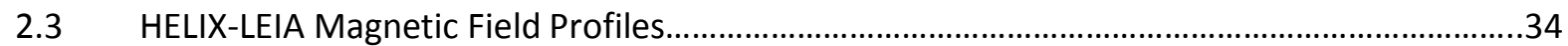

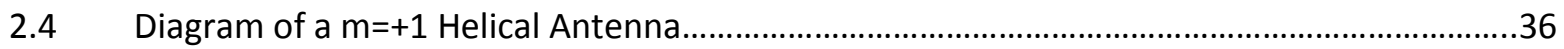

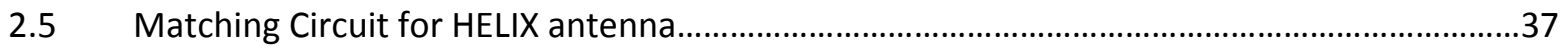

Chapter 3 Standard Plasma Diagnostics

3.1 Idealized Langmuir Probe I-V Characteristic..............................................................................45

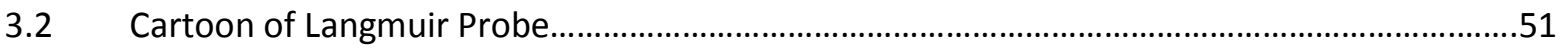

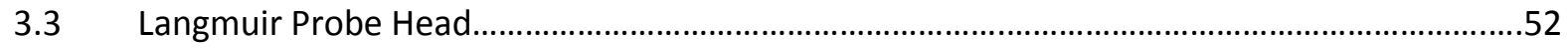

3.4 Langmuir Probe Measurement Circuit......................................................................................53

3.5 End view of the Electrostatic Double Probe..............................................................................5

3.6 Possible Electrostatic Probe Orientations.................................................................................

3.7 Illustration of Spatial Aliasing for a pair of Fixed Probes..........................................................59 
3.8 High Speed Differential Amplifier......................................................................................60

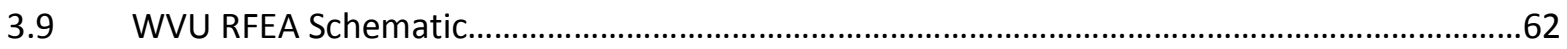

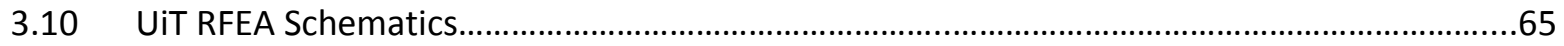

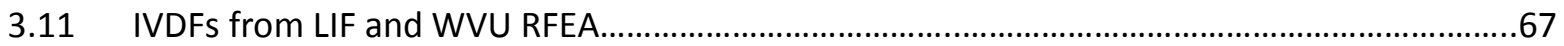

3.12 $\sigma$ and $\pi$ Transitions $611.6616 \mathrm{~nm}$ Argon Ion Absorption Line...............................................70

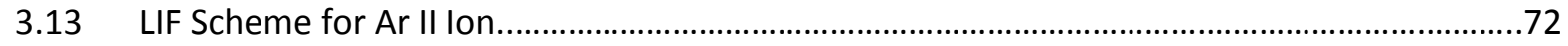

3.14 The Continuous Wave LIF Diagnostic Apparatus................................................................... 73

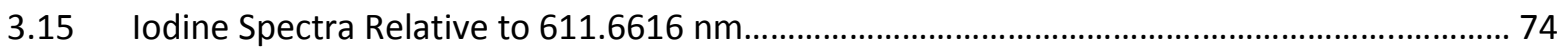

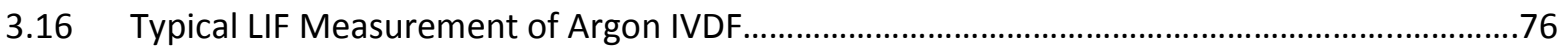

3.17 Mechanical Drawing of the UCLA Tilting Port.........................................................................77

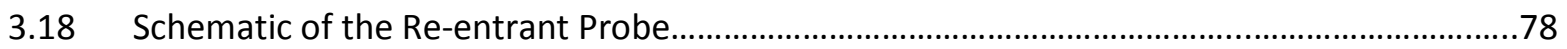

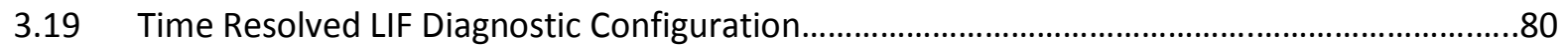

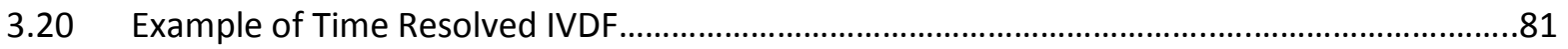

Chapter 4: Signal Processing Techniques

4.1 Power Spectra versus Antenna Frequency..................................................................................

4.2 Illustration of the Short Time Fourier Transform on a Test Signal...........................................99

4.3 Time Frequency Windows used in STFT and Wavelet Transforms.....................................101

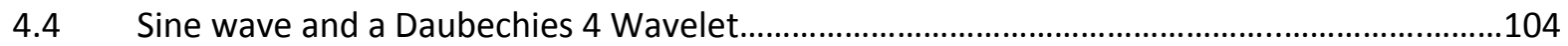

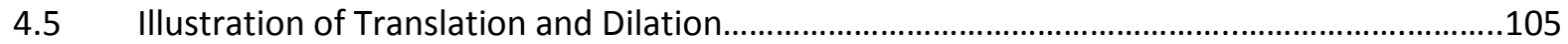

4.6 Examples of Four Different Wavelet Functions.................................................................110

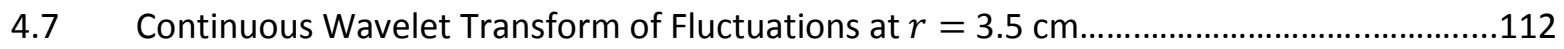

4.8 Spectral Power versus Frequency during a 5 ms Sampling Window.......................................113

Chapter 5: Signal Processing Techniques

$5.1 \quad$ Time Resolved IVDF 9.0 MHz, Mirror Ratio of 60, 5.5 ms................................................118

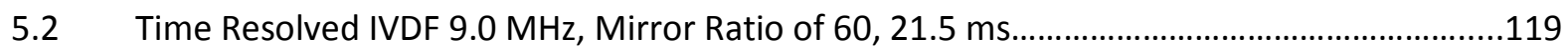


5.3 Time Resolved IVDF 9.0 MHz, Mirror Ratio of 30, 25.5 ms...............................................120

5.4 IVDF versus Time for 9.0 MHz Mirror Ratio Cases of 60 and 30.........................................122

5.5 IVDF versus Time for $9.0 \mathrm{MHz}$ Mirror Ratio Cases of 44 and 22........................................125

5.6 2D Plot of IVDF versus Time for Mirror Ratio Cases 44 and 22...........................................126

5.7 Cross Power Spectrum of 2 Tips of the Electrostatic Probe and no Wave..........................128

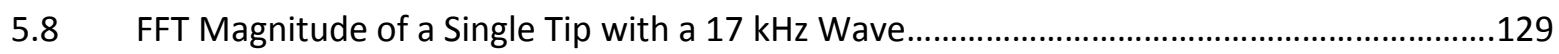

5.9 Cross Power Spectrum with Beam LIF Signal and a Probe Tip............................................130

5.10 Cross Power Spectrum with Background LIF Signal and a Probe Tip...................................131

5.11 Continuous Wavelet Transform of a Single Time Series from Probe Tip.............................133

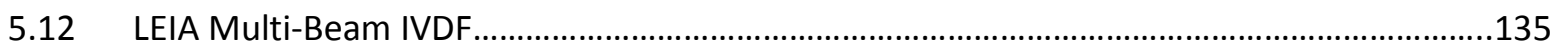

5.13 LEIA Double Layer IVDF at Three Different Axial Positions..................................................137

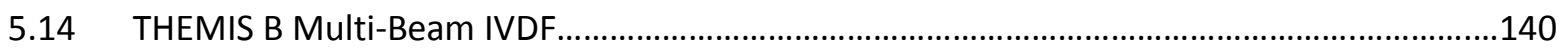

5.15 UC-Boulder PIC Simulation Multi-Beam IVDF....................................................................142

5.16 MST Data Re-plotted with scaling from Random Electric Fields..........................................145

5.17 LEIA Double Layer IVDF at Five Different Axial Positions........................................................147

5.18 Comparison of IVDFs from LIF and UiT RFEA ...................................................................

5.19 LIF Data Presented in the Form of Ideal RFEA Measurements...........................................150 


\section{Chapter 1: Introduction}

The research presented here focuses on double layers (DL) which have been shown to occur in both space and laboratory plasma. The goal of this investigation was to conduct additional studies on double layer formation. Fields such as space propulsion have benefited from past DL research with the invention and validation of the helicon double layer thruster (HDLT). ${ }^{1}$ Still, challenges remain that stem from not fully understanding the mechanism of DL generation. The observation of $U$ shaped potentials in an expanding helicon device ${ }^{2,3}$ implies that one dimensional models of DL formation must be replaced entirely with multi-dimensional models. ${ }^{4}$ Current free double layer (CFDL) research continues to provide opportunities for the plasma physics community to expand its understanding of sheath-related physics.

This dissertation encompasses both the development and enhancement of diagnostic methods as well as explorations of fundamental physics. Instabilities that govern the appearance of a $\mathrm{DL}^{31}$ (as evidenced by the presence of an accelerated beam of ions) were explored in greater detail with new time-frequency analysis methods. A new, modular, retarding field energy analyzer (RFEA) probe was also benchmarked, enabling DLs to be studied without the issues associated with metastable quenching that interfere with laser induced fluorescence techniques.

These investigations also raised questions regarding previous observations in space plasmas where multiple accelerated ion populations are typically attributed to magnetic reconnection. These observations suggest that observations of ion heating during magnetic reconnection may not be evidence of true irreversible heating and might, instead, reflect 
averaging over a complex collection of double layers. ${ }^{5}$ Thus, more caution must be used when analyzing ion velocity distribution measurements from systems in which magnetic reconnection occur in regions of divergent magnetic fields.

The rest of this chapter provides an overview of double layers with a particular focus on current free double layers (CFDLs). Relevant observations of CFDLs in both space and laboratory are discussed in preparation for explaining the new observations presented in Chapter 5. For example, the THEMIS satellite array, which is designed to study substorms in the Earth's magnetosphere, recently detected the presence of DLs in the plasma sheet. In the same region, complex ion beam structures are observed by plasma instruments aboard THEMIS. These ion beam structures are currently attributed to magnetic reconnection events which are identified in magnetic field measurements. Our laboratory observations will show that caution should be applied when interpreting complex ion beam structures as evidence of magnetic reconnection if other substantiating measurements are unavailable. Chapter 3 covers the diagnostics used to gather the data while Chapter 4 provides a detailed description of the signal processing techniques employed for time resolved measurements. A summary of the major results and suggestions for future work are presented in Chapter 6.

\subsection{Double Layers}

In its simplest form, a double layer (DL) consists of two spatially separated charge layers, one positive and one negative. A DL acts very much like a sheath. However, whereas a conventional sheath appears at the surface of an object inserted into the plasma or at the plasma boundary, a DL is a freestanding structure that can appear anywhere within the plasma. Figure 
1.1 shows a schematic of a DL from Block’s review article on double layers. ${ }^{6}$ Note that even in quasi-neutral plasma, quasi-neutrality is violated within the DL. Whereas a sheath at a boundary is roughly a Debye length thick, DL thicknesses are predicted to be 10 - 50 times the Debye length. ${ }^{6}$ The Debye length is a measure of the shielding distance or thickness of a sheath and is defined as

$$
\lambda_{D} \equiv\left(\frac{\epsilon_{o} k_{B} T_{e}}{n e^{2}}\right)^{1 / 2}
$$

where $\epsilon_{o}$ is the permittivity of free space, $k_{B}$ is the Boltzmann constant, $T_{e}$ is the electron temperature, $n$ is the plasma density, and $e$ is elementary unit of charge. ${ }^{7}$ DLs often separate regions of plasma with widely different densities and temperatures and are an important mechanism for the acceleration of charged particles along magnetic fields in laboratory and astrophysical plasmas. 

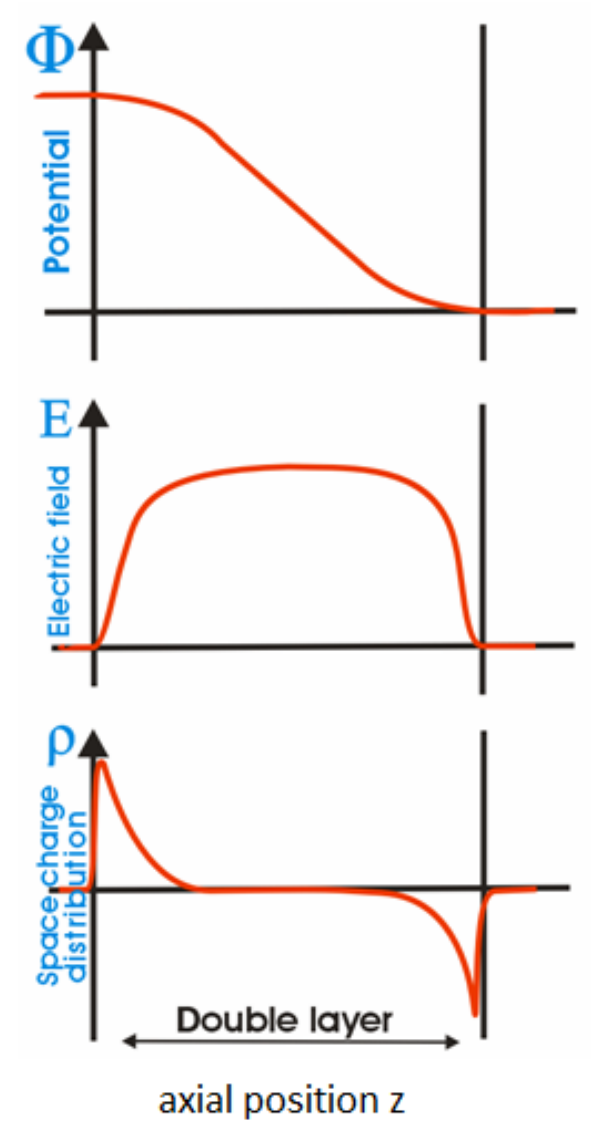

Figure 1.1. A cartoon showing the potential, electric field and space charge through a double layer. Figure obtained from Ref. [6].

Since 2002, several laboratory experiments have reported observations of spontaneous formation of current free double layers (CFDLs) in expanding plasmas with a diverging magnetic field. ${ }^{8,9,10,11,12}$ These DLs are considered "current free" because no net current is injected into the plasma. Instead, the DL spontaneously appears at low pressures in divergent magnetic field regions.

Through decades of in-situ measurements, CFDLs or other magnetic field aligned electric fields have been identified as a source of precipitating electrons ${ }^{13}$ and upwelling ions ${ }^{14}$ in the 
Earth’s magnetosphere. In space and laboratory measurements, evidence for a CFDL is provided by a population of accelerated ions or electrons or direct measurements of the electric potential structure. In ion energy distribution function measurements by spacecraft, CFDLs have been identified in magnetospheric regions ranging from the auroral zone to the plasma sheet. ${ }^{15}$

DL review articles often focus on specific sub-topics within the broader DL research area. Raadu ${ }^{16,17}$ for example, addressed the basic physics of DLs through laboratory measurements and noted implications for space and astrophysical plasmas such as the presence of instabilities in both. Hershkowitz's review ${ }^{\mathbf{1 8}}$ focused on early laboratory experiments. Elizer and Hora $^{19}$ focused on rarefaction shocks. Charles et al. ${ }^{20}$ reviewed additional laboratory measurements that emphasized the new discovery of CFDLs in expanding helicon plasma devices. Singh's very recent review ${ }^{4}$ focused on explaining basic plasma processes found in CFDL formation while also discussing significant laboratory experiments, simulations and space observations.

\subsection{Space Observations of Current Free Double layers}

Recent measurements from the THEMIS satellites have established the prevalence of double layers in the plasma sheet. The plasma sheet, shown in Fig. 1.2, is a relatively speaking high density collisionless plasma region in the ecliptic at the earthward end of the magnetotail. ${ }^{21}$ Ion temperatures in the plasma sheet are $\sim 5 \times 10^{7} \mathrm{~K}$. Fig. 1.3 shows the location of the THEMIS (Time History of Events and Macroscale Interactions during Substorms) probes in the Earth’s magnetosphere. The THEMIS mission, which includes five identical satellites, was designed to examine the nature of the impulsive events that release solar wind energy stored within the 
Earth's magnetotail. ${ }^{22}$ Orbit apogees of the satellites (in units of Earth radii, $R_{E}$ ) are 10 (probe A), 12 (probes D and E), 20 (probe C), and 30 (Probe B). Each spacecraft is equipped with electron and ion analyzers, a three-axis electric field instrument, and magnetometers.

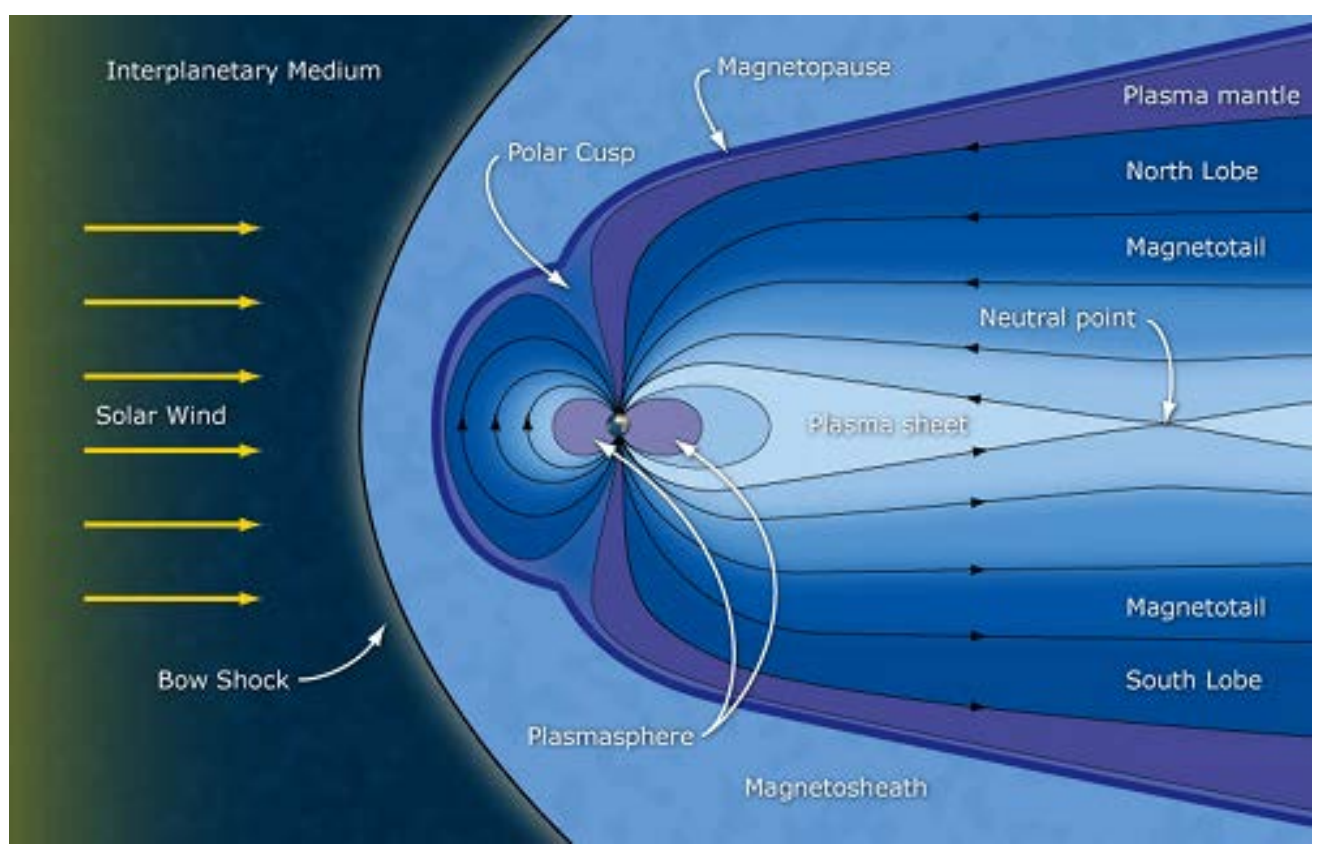

Figure 1.2. Cartoon of the Earth's magnetosphere. Note the location of the plasma sheet. Figure courtesy of ESA/C.

T. Russell. 


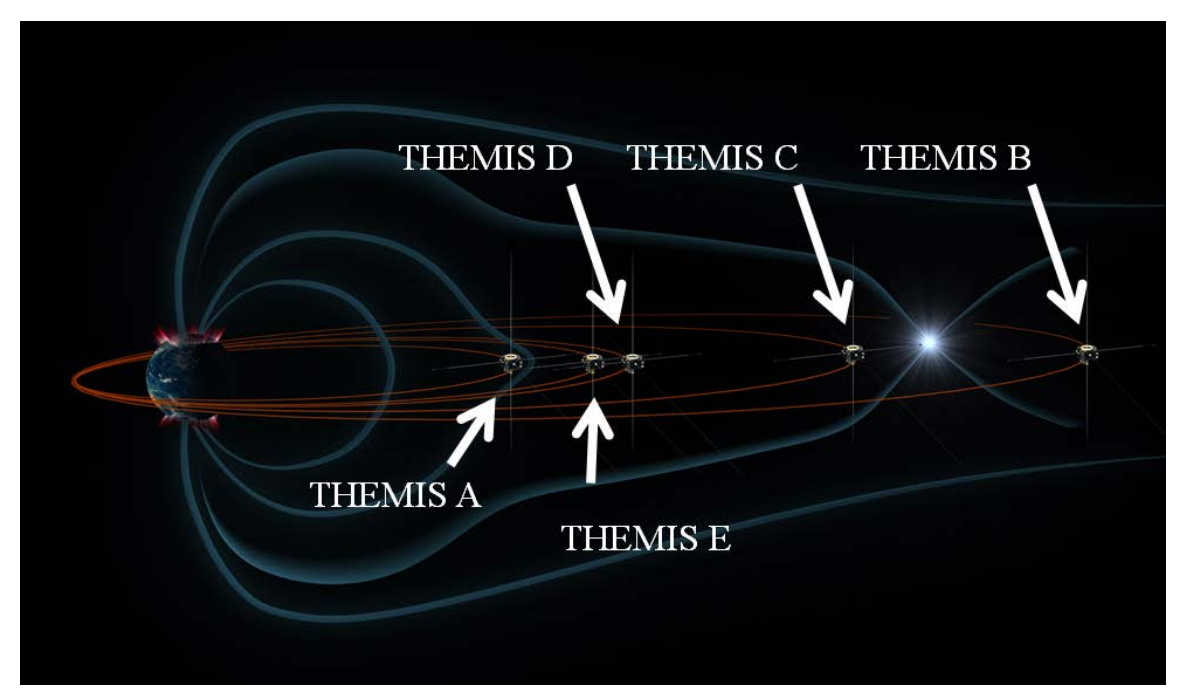

Figure 1.3. THEMIS satellites in their "aligned” configuration. Note THEMIS B's position on the tailside of the reconnection site. (Courtesy of NASA).

Evidence for DLs in the plasma sheet is shown in Fig 1.4a-1.4d. The data show two bursty bulk flow events recorded on 24 Mar 2003 with THEMIS Probe D. Event 1 was recorded at 8192 samples/s, the high time resolution setting (Fig 1.4a-1.4c). Event 2 was recorded at only 128 samples/s (Fig 1.4d). The parallel component of the electric field, $E_{\|}$, and two components of the perpendicular electric field are shown in Fig 1.4a-1.4c as measured with three orthogonal dipole antennas. The $E_{\|}$measurement shows strong turbulence from -0.05 to 0.10 seconds, followed by a smooth ramping to a constant electric field. The constant value of $E_{\|}$persists from 0.12 to 0.14 seconds. Ergun et al. (2009) refer to the unipolar $E_{\|}$structure adjacent to a turbulent region as a "signature" and points out that this signature is identical to those identified as double layers in the auroral ionosphere. ${ }^{15}$ 

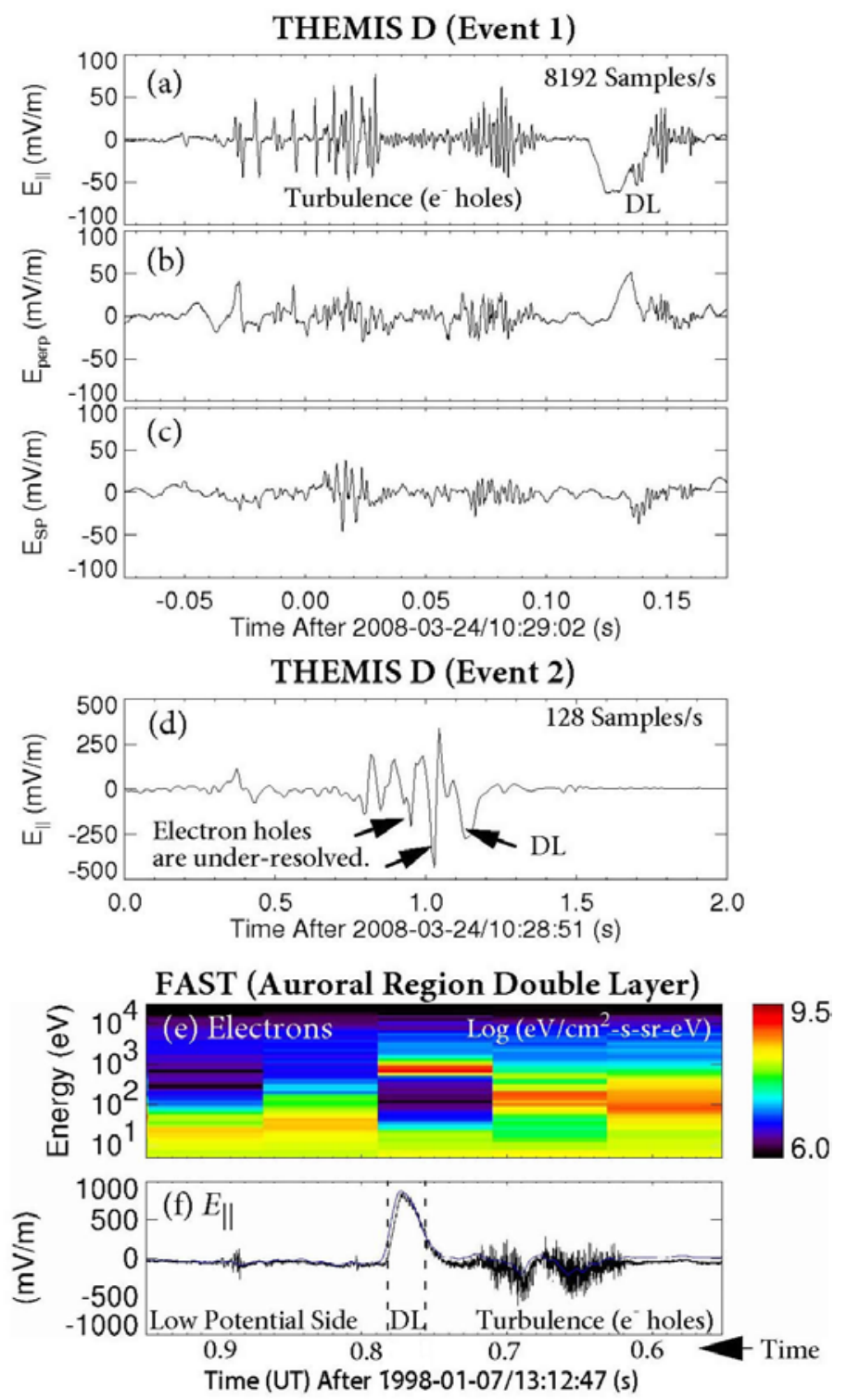

Figure 1.4. (a) Parallel electric field sampled at $8192 \mathrm{~Hz}$ during Event 1. (b) and (c) are the perpendicular electric field. (d) Parallel electric field sampled at $128 \mathrm{~Hz}$ during Event 2. (e) and (f ) are the electron energy flux and parallel electric field of an auroral DL sampled at $32768 \mathrm{~Hz}$. Figure adapted from Ref. [15].

Fig. 1.4e-1.4f is a measurement of a DL in the auroral ionosphere obtained by the FAST (Fast Auroral SnapshoT) spacecraft for comparison to the plasma sheet data. The FAST satellite measured electromagnetic fields and charged particle distributions in the earth’s auroral zone. ${ }^{23}$ 
The FAST satellite was in a near-polar orbit with an $83^{\circ}$ inclination, a $350 \mathrm{~km}$ perigee, and a $4175 \mathrm{~km}$ apogee. The FAST satellite instrument complement included electron and ion spectrographs, 3-axis electric and magnetic field instruments. ${ }^{24}$ The electric field instruments detected an electron beam (Fig. 1.4e) consistent with a double layer-like potential structure (Fig 1.4f), confirming the presence of an auroral double layer. The THEMIS electric field observations in the plasma sheet display the same characteristics.

Ergun et al. (2009) then deduced the detection of tens of DLs throughout the plasma sheet in spite of the fact that THEMIS only has the ability to record with highest resolution for only $0.05 \%$ of the orbit. Previous researchers had expected that the observation of DLs should be statistically rare since the DLs occupy a very small spatial volume in the magnetosphere. Thus, Ergun et al. (2009) concluded that DLs occur frequently in the plasma sheet during magnetic activity. ${ }^{15}$

\subsection{Laboratory Studies of Double Layers}

Nearly all recent laboratory DL experiments have been performed in helicon plasma devices. As stated previously, Charles et al. ${ }^{20}$ and $\operatorname{Singh}^{4}$ provide a thorough review of recent CFDL experiments. Chakraborty Thakur provides a thorough review of DL experiments prior to

2010 at WVU. ${ }^{25}$ Here a few recent developments in laboratory DL experiments are reviewed as well as one of the theoretical models proposed as an explanation for the laboratory DL observations. The focus will be on experimental results along with a crude model that does not completely explain our multiple DL laboratory observations. However, the model does offer a possible explanation for the source of multiple double layers and the analysis of the 
measurements includes suggestions for future investigations that might more clearly identify the physical processes that create the complex ion velocity distribution functions (IVDFs).

\subsubsection{Current Free Double Layer Observations at ANU}

Having obtained some of the first measurements of CFDLs in the laboratory over a decade ago, the Space Plasma, Power and Propulsion Group, at the Australian National University (ANU) recently moved on to DL experiments designed to investigate similar DL geometries as those explored by the FAST satellite. ${ }^{2,3}$ A schematic of their experimental device known as CHI KUNG is shown in Fig. 1.5. The CHI-KUNG sketch provides a general sense of how helicon plasma sources with expansion regions are configured. ${ }^{3}$ The dimensions vary by device (WVU helicon source is much larger). The expansion geometry sets up an abruptly diverging magnetic field after a uniform magnetic field in the source region.

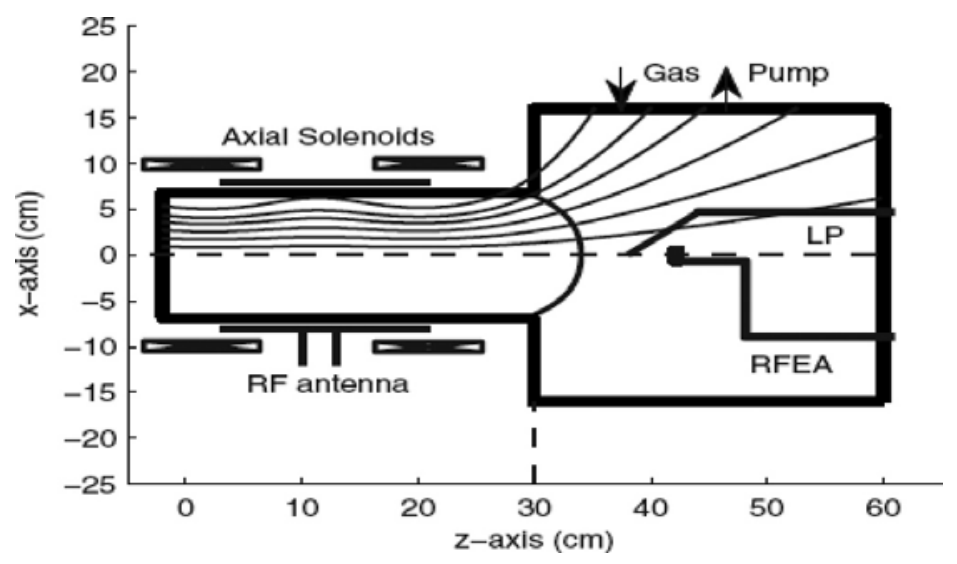

Figure 1.5. Schematic of the CHI KUNG expanding plasma device with diverging magnetic field lines. Diagnostics include the RFEA and rf compensated Langmuir probe. The parabola shown by a solid line near the exit of the plasma source is the low potential edge of the DL. Figure obtained from Ref. [3]. 
Shown in Fig 1.6a is the auroral model used to evaluate the path of the FAST satellite as it travelled through the downward current region. The downward current region is characterized by parallel electric fields which produce anti-earthward energetic electron fluxes (up to several $\mathrm{keV}$ ) carrying the "downward" current in the auroral zone. ${ }^{24}$ Fig. $1.6 \mathrm{~b}$ shows the experimental configuration used by the ANU group. The probe path through the DL in the divergent magnetic field at the end of the source is analogous to the path of the satellite. The U shaped potential structure identified by Ergun et al. (2003) demonstrated that DLs can exist in weakly converging magnetic fields. For the ANU experiments, a retarding field energy analyzer (RFEA) probe, described in greater detail in Chapter 3, mapped out the ion beam current and the plasma potential in the plasma. With the RFEA, the ANU group obtained the 2D equipotential and ion density contours shown in Figure 1.7. The double layer extends between the $46 \mathrm{~V}$ and $36 \mathrm{~V}$ contours in Fig 1.7a with the red line providing a contour fit to the low potential side. The ion density profile (Fig. 1.7b) shows that the areas of greatest density are located along the most divergent magnetic field line. Thus, the ANU laboratory experiments confirmed that a U shaped DL structure can be created in the laboratory with a weakly diverging magnetic field. 
A) Space

Magnetosphere
B) Laboratory

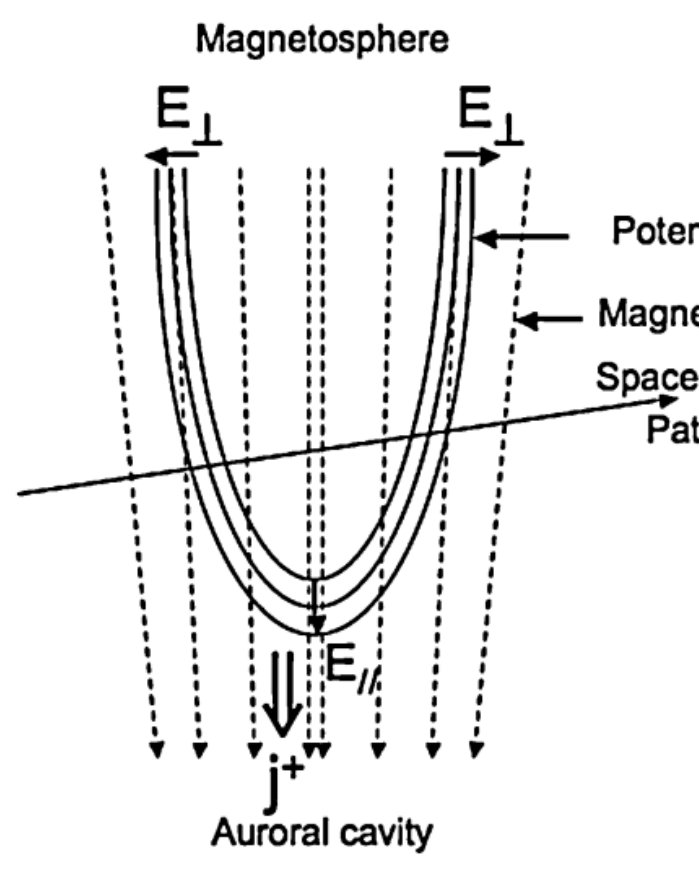

Plasma source
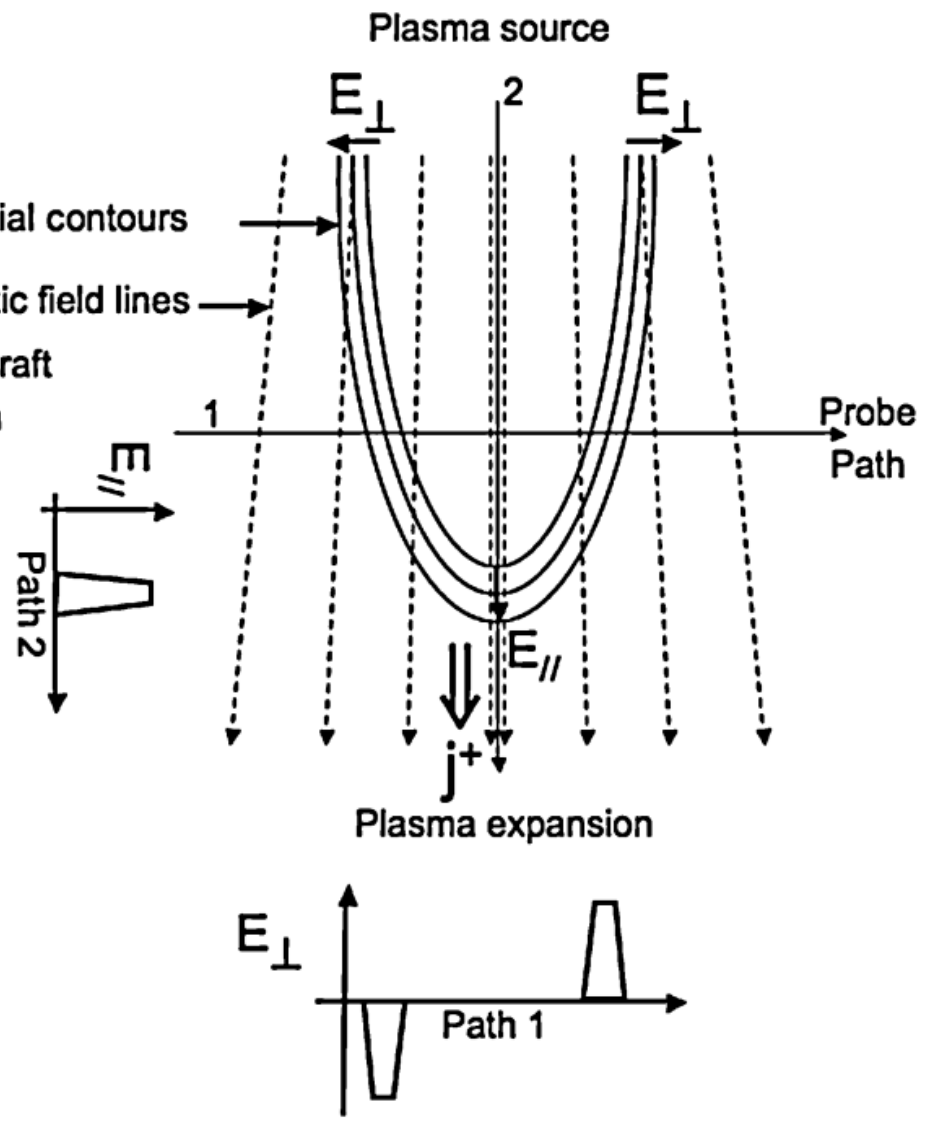

Figure 1.6. A schematic interpreting the observations from (a) the FAST satellite traveling through the downward current region. $j^{+}$represents the downward accelerated ion current (b) The laboratory probe traversing the experimental double layer. Figure obtained from Ref. [2]. 
(a)
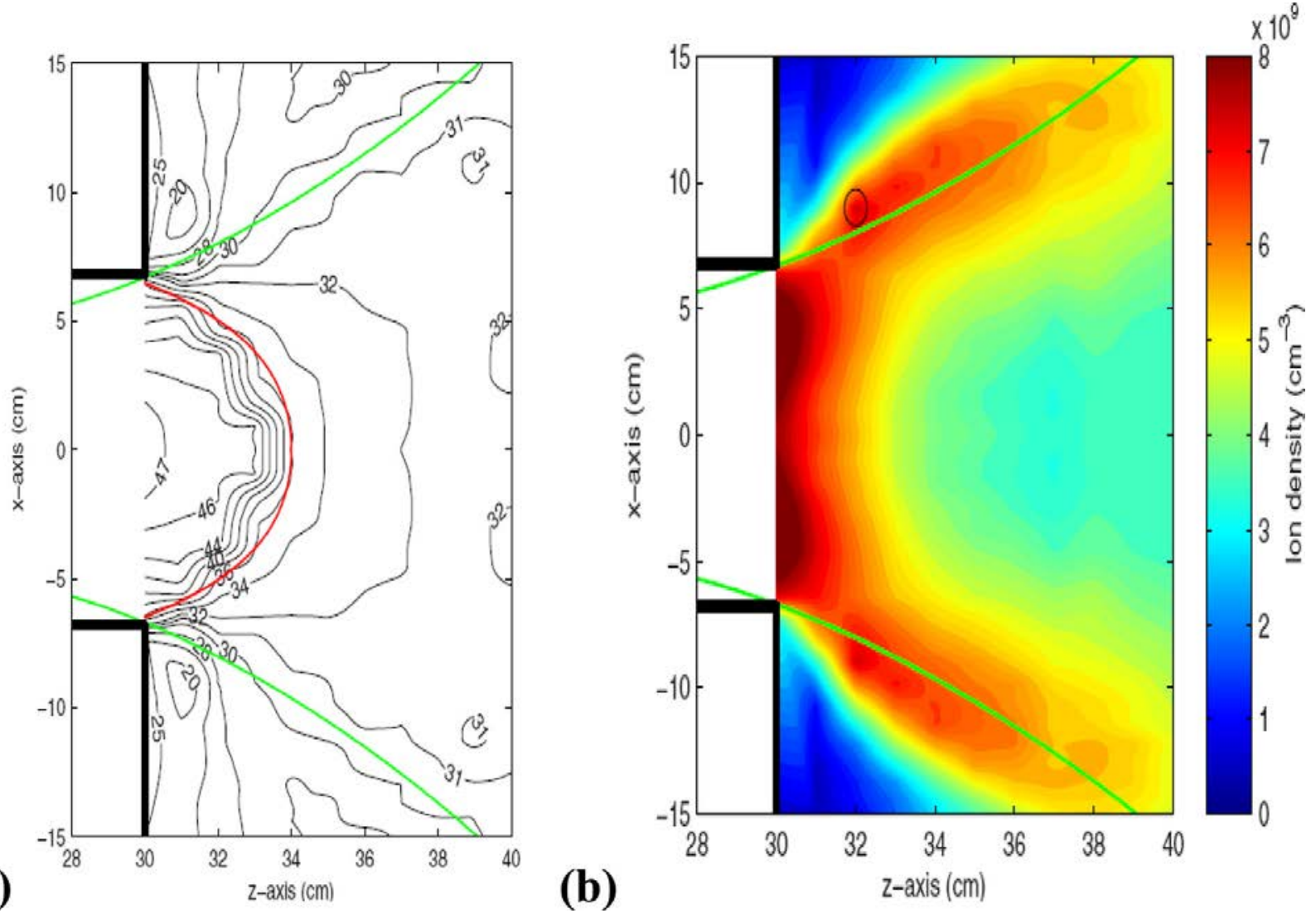

Figure 1.7. (a) 2D equipotential contours measured with the RFEA. The DL extends between the 36 and $46 \mathrm{~V}$ contours. The solid parabolic line represents a fit of the $36 \mathrm{~V}$ contour which is the low potential edge of the Ushaped current-free double layer. The solid diverging line shows the most diverging magnetic field line exiting the source. (b) 2D contours of the ion density measured with the RFEA. Figure obtained from Ref. [3,4].

\subsubsection{CFDLs in Expanding Helicon Plasmas Theory}

The one dimensional DL theory created by Lieberman and Charles was proposed to explain the early one-dimensional CFDL measurements. ${ }^{26,27}$ Their diffusion-controlled model coupled the dynamics of the particles in the non-neutral DL to the diffusive flows of the quasineutral plasma in the source and expansion chambers. To ensure that the DL was current-free, in 
addition to the conventional four DL populations described by Andrews and Allen ${ }^{28}$ the model required another population of counter-streaming electrons, formed by the reflection of almost all of the accelerated electrons from the sheath at the insulated end wall of the source chamber. Several published accounts from the WVU Helicon Plasma Group have reported increased levels of upstream ionization during the observation of a DL consistent with this theory. ${ }^{25,29}$

Singh argues in his review article that this diffusion controlled model is of limited validity because while it may be able to explain the parallel potential drop in the observed $U$ shaped double layer, it does not explain the perpendicular potential drop. ${ }^{4}$ Singh bases his argument on an analysis of magnetization and transit times. A particle's gyromotion about a magnetic field line is describable with a cyclotron motion of period

$$
\tau_{s}=\frac{2 \pi}{\omega_{c s}}=\frac{2 \pi m_{s}}{\left|q_{s}\right| B}
$$

and radius, the Larmor radius, of

$$
\rho_{s}=\frac{m_{s} v_{\perp}}{\left|q_{s}\right| B}
$$

where $s$ denotes species, $\omega_{c s}=\frac{\left|q_{s}\right| B}{m_{s}}$, is the cyclotron frequency of the denoted species, $m$ is the mass, $q$ is the charge of the species, $B$ is the magnetic field strength and $v_{\perp}$ is the velocity component perpendicular to the magnetic field (in this case the thermal velocity). ${ }^{30}$ 
Using data provided by Charles et al., 2,3 Singh compares the time it takes to transverse the parallel DL to the cyclotron period, a measure of how much influence the background magnetic field has on a particle. For the CHI KUNG experiment, the ions are unmagnetized and the electrons are highly magnetized. The difference in magnetization implies that when the electrons exit the source, they will quickly follow the diverging magnetic field, and then attract ions through a self-consistent perpendicular electric field, setting up the large conical structure shown in Fig. 1.7b.

Singh argues that when perpendicular electric fields develop near density gradients due to differing electron and ion Larmor radii, the perpendicular electric fields are shorted out by conducting boundaries in a laboratory plasma. The resulting parallel electric field may then be localized at a single DL or be spread out across multiple DLs. Singh makes the claim that the perpendicular electric field is the source of the potential drop that drives the CFDL in an expanding helicon source. ${ }^{4}$

For the WVU expanding helicon device during typical DL studies, the upstream magnetic field is $7{\mathrm{X} 10^{-2}}$ Tesla, the thermal temperatures are $T_{i} \sim 0.2 \mathrm{eV}$ and $T_{e} \sim 0.6 \mathrm{eV}$, and the parallel scale length of the DL is $\sim 30 \mathrm{~cm}$. Using this information along with the mass of the argon ion and an electron in Eq. 1.2 and Eq. 1.3, the cyclotron period for the ions and electrons are $\tau_{c i} \sim 37$ $\mu \mathrm{s}$ and $\tau_{c e} \sim 0.5 \mathrm{~ns}$ respectively. From the temperatures, the thermal velocities for the ions and electrons are $\sim 690 \mathrm{~m} / \mathrm{s}$ and $\sim 10^{6} \mathrm{~m} / \mathrm{s}$, giving parallel double layer transit times $\tau_{\text {i trans }} \sim 430 \mu \mathrm{s}$ and $\tau_{\text {e trans }} \sim 290$ ns. Unlike the CHI KUNG experiment, both ions and electrons are considered magnetized but the electrons are much more magnetized, $\left(\tau_{c i}<\tau_{i \text { trans }}\right.$ and $\tau_{c e}<<\tau_{e}$ trans $)$. The fact that both species are magnetized in the WVU helicon device mitigates Singh's claim that the 
perpendicular electric field is the driving force to all CFDL helicon sources. The ions are likely to follow the magnetic field lines into the double layer along with electrons. Consequently, the Lieberman and Charles diffusion controlled model used by Chakraborty Thakur et al. to explain upstream ionization has more validity in WVU expanding helicon plasma source. What is also true, however, is that the ion Larmor radius, $\left(\rho_{i} \sim 4 \mathrm{~mm}\right.$ at the beginning of the DL, $\sim 10 \mathrm{~cm}$ at the low potential side) may be substantial enough for the ions to sample some of these other nonparallel potentials that may be present in the DL.

\subsubsection{Double Layer Laboratory Studies at WVU}

The WVU Helicon Plasma Group has also made substantial contributions to CFDL laboratory research. Chakraborty Thakur ${ }^{25}$ and Scime et al. ${ }^{31}$ provide a thorough review of previous WVU research. Sun et al. ${ }^{32}$ provided the first published observation of supersonic ion flows in WVU's expanding helicon experiment and was followed by more detailed observations of a CFDL that are described in Ref. [10]. This present work finds its inspiration largely in trying to go beyond the studies published in Biloiu et al. ${ }^{33}$ and Chakraborty Thakur et al. ${ }^{29} \mathrm{~A}$ brief synopsis of those studies will be given here, with specific facets reviewed in the relevant portions of Chapter 5.

Chakraborty Thakur et al. ${ }^{29}$ performed a series of pivotal experiments that investigated the effect of only changing the antenna frequency on the formation of the ion beams downstream of a low pressure expanding helicon argon plasma. All other source parameters such as the magnetic field in the source and expansion chambers, the power supplied to the driving antenna, and the neutral gas pressure were held fixed. The velocity of the ion beam in the source 
(upstream, black squares) and in the diffusion chamber (downstream, red circles) is shown as a function of the antenna frequency in Fig. 1.8. Above the antenna frequency threshold of 11.5 $\mathrm{MHz}$, the ion beam appears downstream of the plasma source and the beam velocity decreases with increasing driving frequency. The decrease in downstream ion beam velocity with increasing driving frequency suggests that the ion beam velocity would be even larger at lower driving frequencies if whatever mechanism that prevents ion beam formation did not appear at the antenna frequency of $11.5 \mathrm{MHz}$. An electrostatic double probe was also used to measure the frequency spectrum of the electric field fluctuations for plasmas with and without a stable double layer. The electrostatic fluctuation measurements pointed to a beam-driven, ion acoustic instability as the mechanism responsible for suppression of the DL at low antenna frequencies. ${ }^{29}$

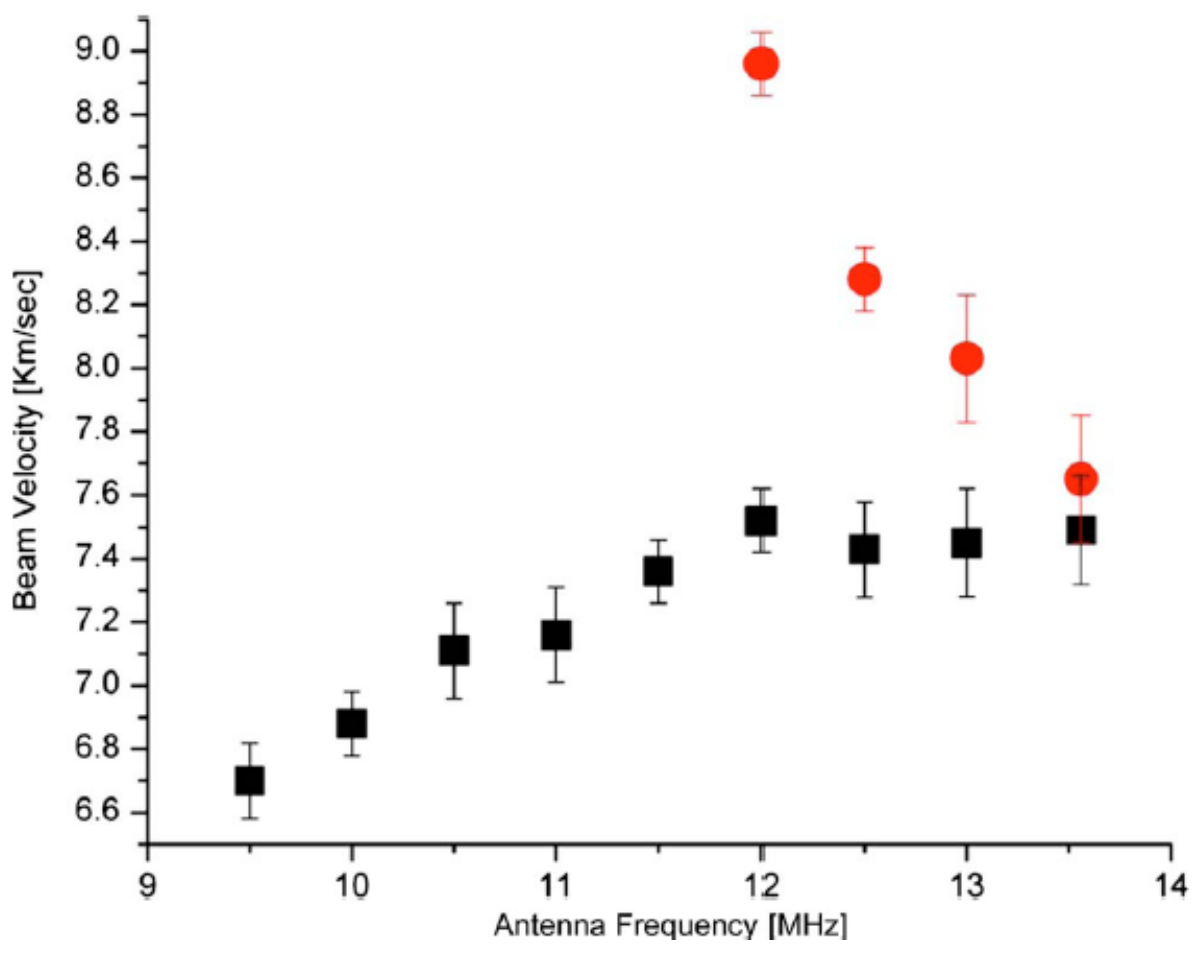


Figure 1.8. Upstream (black squares) and downstream (red circles) ion beam velocity versus antenna frequency. The velocities have been corrected for Zeeman shifts and the angle of the downstream LIF probe. The downstream ion beam vanishes for lower antenna frequencies. Figure obtained from Ref. [29].

We suggest the following interpretation of these observations. At antenna frequencies below 11.5-12 MHz, a strong DL forms and accelerates an ion beam to velocities greater than the sound speed. The accelerated ion and electron beam currents exceed a threshold for excitation of a current-driven instability and large electrostatic fluctuations develop; thereby destroying the strong potential gradient necessary for the DL and the DL collapses. The instabilities appear as large amplitude noise on Langmuir probe and RFEA measurements in steady-state discharges. The DL is stronger (the relative intensity of the ion beam is larger and the ion beam velocity is larger) at lower antenna frequencies because the coupling of rf energy into the plasma improves at lower antenna frequencies. Thus, it is at the higher antenna frequencies that the ion beam persists in both the pulsed and steady-state discharges.

Once the rf power coupling efficiency drops at higher antenna frequencies, a stable, but weaker, DL forms; the electrostatic noise is significantly reduced; and the ion beam appears downstream. Consistent with this hypothesis are the measurements of the upstream bulk ion speeds and the downstream ion beam velocities shown in Fig. 1.8 The downstream ion beam velocity clearly increases with decreasing antenna frequency (the DL is getting stronger) until the beam abruptly vanishes downstream. The upstream beam velocity is relatively constant at the higher antenna frequencies and then begins to drop at the same threshold antenna frequency (11 $\mathrm{MHz}$ ) for which the downstream beam vanishes. In the time resolved studies, two ion beam velocity and amplitude cases were obtained through two different divergent magnetic field 
mirror ratios. For the larger beam velocity and amplitude case, the instability grows while the DL collapses (the ion beam vanishes). For smaller beam velocity and relative amplitude case, the ion beam persists throughout the discharge and no electrostatic fluctuations are observed; consistent with the higher beam velocity results obtained in the steady-state discharge experiments.

Because the growth of the instability disrupts the DL, these measurements provide a unique means of experimentally studying the physics related to the formation of a current-free DL in expanding plasmas. These experiments are not the first to indicate the presence of low frequency instabilities associated with current-free $\mathrm{DLs}^{34}$ or with DLs created in divergent magnetic fields. ${ }^{35}$ However, to the best of our knowledge, complete collapse of a DL correlated with the appearance of intense electrostatic instabilities had not been reported previously in a laboratory experiment. These results suggested that creation of strong DLs in expanding plasmas for plasma propulsion ${ }^{36,37}$ may be self-limited through instability growth and also demonstrated a mechanism for the collapse of naturally occurring DLs. The nature of this instability is discussed in greater detail in Section 4.1 and additional results and analysis motivated by this work are presented in Section 5.1. 


\section{Chapter 1 References}

${ }^{1}$ C. Charles, R. Boswell, P. Alexander, C. Costa, and O. Sutherland, L. Pfitzner, R. Franzen, J. Kingwell, A. Parfitt, P.-E. Frigot, J. Del Amo, and G. Saccocia, IEEE Trans. Plasma Sci., 36, 1196 (2008).

${ }^{2}$ C. Charles, R. W. Boswell, R. Hawkins, Phys. Rev. Lett. 103, 095001 (2009).

${ }^{3}$ C. Charles, Appl. Phys. Lett. 96, 051502 (2010).

${ }^{4}$ N. Singh, Phys. Plasmas 18, 1 (2011).

${ }^{5}$ J. Carr, Jr., P. A. Cassak, M. Galante, A. M. Keesee, G. Lusk, R. M. Magee, D. McCarren, E. E. Scime, S. Sears, R. Vandervort, N. Gulbrandsen, M. Goldman, D. Newman, and J. P. Eastwood, Phys. Plasmas 20, 072118 (2013).

${ }^{6}$ L. P. Block, Astrophys. And Space Sci. 55, 59 (1978)

${ }^{7}$ F.F. Chen in Introduction to plasma physics and controlled fusion: Volume 1, (Springer Science and Business Media, LLC, 2006).

${ }^{8}$ S. A. Cohen, N.S. Siefert, S. Stange, R.F. Boivin, E.E. Scime, and F.M. Levinton, Phys. Plasmas 10, 2593 (2003).

${ }^{9}$ C. Charles and R. W. Boswell, Appl. Phys. Lett. 82, 135 (2003).

${ }^{10}$ X. Sun, A. M. Keesee, C. Biloiu, E. E. Scime, A. Meige, C. Charles C and R. Boswell Phys. Rev. Lett. 95, 025004 (2005).

${ }^{11}$ N. Plihon, C. S. Corr, and P. Chabert, Appl. Phys. Lett. 86, 091501 (2005).

${ }^{12}$ K. Takahashi, C. Charles, R. Bowell, and R. Hatakeyama, Phys. Plasmas 15, 074505 (2008).

${ }^{13}$ R. D. Albert and P. J. Lindstrom, Science 170, 1398 (1970). 
${ }^{14}$ D. Schriver, M. Ashur-Abdalla, R.J. Strangeway, R.L. Richard, C. Klezting, Y. Dotan, J. Wygant, J. of Geophys. Res., 108, COA21 (2003).

${ }^{15}$ R. E. Ergun, L. Andersson, J. Tao, V. Angelopoulos, J. Bonnell, J.P. McFadden, D.E. Larson, S. Eriksson, T. Johansson, C.M. Cully, D.N. Newman, M.V. Goldman, A. Roux, O. LeContel, K.-H. Glassmeier, W. Baumjohann, Phys. Rev. Lett. 102, 155002 (2009).

${ }^{16}$ M. A. Raadu, Astrophys. Space Sci. 144, 43 (1988); Phys. Rep. 178, 25 (1989).

${ }^{17}$ M. A. Raadu, Space Sci. Rev. 68, 29 (1994).

${ }^{18}$ N. Hershkowitz, Space Sci. Rev. 41, 351 (1985).

${ }^{19}$ S. Eliezer and M. Hora, Phys. Rep. 172, 339 (1989).

${ }^{20}$ C. Charles, Plasma Sources Sci. Technol. 16, R1 (2007).

${ }^{21}$ K. Seki, M. Hirahara, M. Hoshino, T. Terasawa, R. C. Elphic, Y. Saitok, T. Mukaik, H. Hayakawak, H. Kojima and H. Matsumoto Nature, 422, 589 (2003).

${ }^{22}$ V. Angelopoulos, Space Sci. Rev. 141, 5 (2008).

${ }^{23}$ C. W. Carlson, R.F. Pfaff, J. G. Watzin, Geophys. Res. Lett. 25, 2013 (1998).

${ }^{24}$ R. E. Ergun, L. Andersson, C.W. Carlson, D. L. Newman, and M.V. Goldman, Nonlinear Proc. Geophys. 10, 45(2003).

${ }^{25}$ S. Chakraborty Thakur, Understanding Plasmas through Ion Velocity Distribution Function measurements, Ph. D. Thesis, West Virginia University, Morgantown (2010).

${ }^{26}$ M. A. Lieberman, C. Charles and R. W. Boswell, J. Phys. D: Appl. Phys. 393294 (2006).

${ }^{27}$ M. A. Lieberman and C. Charles, Phys. Rev. Lett. 97045003 (2006).

${ }^{28}$ J. G. Andrews and J. E. Allen, Proc. R. Soc. London A 320, 459 (1971).

${ }^{29}$ S. Chakraborty Thakur, Z. Harvey, I. A. Biloiu, A. Hansen, R. A. Hardin, W. S. Przybysz, and E. E. Scime, Phys. Rev. Lett. 102, $035004 \_2009$. 
${ }^{30}$ P. M. Bellan, in Fundamentals of Plasma Physics, (Cambridge University Press, 2008).

${ }^{31}$ E. E. Scime, I. A. Biloiu, J. Carr, Jr., S. Chakraborty Thakur, M. Galante, A. Hansen, S. Houshmandyar, A. M. Keesee, D. McCarren, S. Sears, C. Biloiu, and X. Sun, Phys. Plasmas 17, 055701 (2010).

${ }^{32}$ X. Sun, C, Biloiu, R. Hardin and E. E. Scime, Plasma Sources Sci. Technol. 13, 459 (2004).

${ }^{33}$ I. A. Biloiu and E. E. Scime, Appl. Phys. Lett. 95, 051504 (2009). 


\section{Chapter 2: Helicon Plasma Sources}

\subsection{Introduction to Helicon Plasma Sources}

Helicon plasma sources have been studied for over forty years with a large spike of research activity occurring within the last 20 years. ${ }^{1}$ The large spike in research activity results from applications that take advantage of the helicon sources' ability to provide low temperature, high density plasmas. Helicons have proven useful in the fields of propulsion, ${ }^{2,3,4,5}$ plasma processing, ${ }^{6,7}$ studying space relevant phenomena, ${ }^{8,9}$ and basic plasma physics. ${ }^{10,11}$

Helicon wave investigations are usually the starting point for any discussions of the generation of helicon plasmas, even though it is unclear what exact mechanism is responsible for coupling rf power into a plasma. Helicon waves were first explored in the early 1960's in gaseous plasmas ${ }^{12}$ and solid systems. ${ }^{13}$ Woods, ${ }^{14}$ Klozenbreg et al., ${ }^{15}$ and Davies et al. ${ }^{16}$ published studies on the basic theory of helicon waves. In the early 1970's, Rod Boswell developed the first helicon plasma source while at Flinders University of Australia. ${ }^{17}$ Boswell observed densities of the order of $10^{13} \mathrm{~cm}^{-3}$ and the signature argon "blue core."18 Boswell and co-workers ${ }^{19,20,21}$ performed several experiments that explored the structure and propagation of helicon waves during the 1980's. The spike of publications began in the early nineties from different groups investigating plasma thrusters, plasma processing, space relevant phenomena, and basic plasma physics. Helicon Double Layer Thrusters (HDLTs) are one example of increased research activity in the field of helicon applications. Takahashi et al. ${ }^{22}$ recently measured the axial thrust of one these devices. Boswell et al. ${ }^{23}$ have concluded more research is needed in environments representative of space, but still the HDLT may prove to be a low-cost, 
long-lifetime, electric propulsion device. Review articles covering the earlier decades of helicon plasma physics research can be found in Boswell and Chen, ${ }^{24}$ Chen and Boswell, ${ }^{25}$ and Scime et al. $^{26}$

\subsection{Physics of Helicon Plasma Sources}

Helicon waves are bounded right handed circularly polarized electromagnetic waves. The frequency range of propagation is $\omega_{c i} \ll \omega \ll \omega_{c e}$ where $\omega_{c i}$ is the ion cyclotron frequency, $\omega_{c e}$ is the electron cyclotron frequency and $\omega$ is the wave frequency. Unbounded or free right-hand circularly polarized electromagnetic waves are commonly referred to as whistler waves because of their characteristic descending tones. ${ }^{27}$ An engineer in the German army named $\mathrm{H}$. Barkhausen reported these tones during World War I. ${ }^{28}$ While eavesdropping on allied communications, he determined that the whistlers were coming from the atmosphere. However, it took the work of Storey ${ }^{29}$ to suggest that these waves are generated by lightning. Aigrain originally coined the term "helicon" in 1960 as a description of bounded right hand circularly polarized waves in a solid rod of sodium. ${ }^{30}$ The dispersion relation for a helicon wave is

$$
N^{2} \approx \frac{\omega_{p e}^{2}}{\omega \omega_{c e} \cos \theta}
$$

Here, $N \equiv k_{\|} c / \omega$, is the parallel index of refraction, $k_{\|}$is the wave number parallel to the magnetic field, $c$ is the speed of light, $\omega_{p e}$ is the electron plasma frequency, $\omega$ is the wave frequency, $\omega_{c e}$ is electron cyclotron frequency and $\theta$ is the angle at which the wave propagates with respect to the magnetic field. Storey also determined that high frequency helicon waves 
travel faster than lower frequency waves emanating from the same source; the group velocity is $(d \omega / d k)_{\max }=\omega / 4 \omega_{c e}$. Thus, helicon waves share the same "whistling" characteristic as whistler waves.

Classic helicon waves have an operating frequency $\omega$ constrained to obey $\omega_{L H} \ll \omega \ll$ $\omega_{c e}$ and $\omega \omega_{c e} \ll \omega_{p e}^{2}$, where $\omega_{L H}$ is the lower hybrid frequency, $\omega_{p e}=\left(n e^{2} / \varepsilon_{o} m_{e}\right)^{1 / 2}$ is the electron plasma frequency, $\omega_{c e}=e B / m_{e}$ is the electron cyclotron frequency and $n, e, \varepsilon_{o}, m_{e}$ and $B$ are the plasma density; elementary electron charge; dielectric permittivity of vacuum; electron mass; and the uniform background magnetic field strength, respectively. The lower hybrid frequency is

$$
\frac{1}{\omega_{L H}^{2}}=\frac{1}{\omega_{p i}^{2}+\omega_{c i}^{2}}+\frac{1}{\omega_{c e} \omega_{c i}}
$$

where $\omega_{p i}=\left(n Z^{2} e^{2} / \varepsilon_{o} M\right)^{1 / 2}$ is the ion plasma frequency, $\omega_{c i}=Z e / M$ is the ion cyclotron frequency, and $Z e, M$ are the ion charge and mass, respectively. The second term contains the effects due to electron inertia. The first term is negligible in higher density plasmas, allowing the lower hybrid frequency to be approximated by $\omega_{L H} \approx \sqrt{\omega_{c e} \omega_{c i}}$ in a high density plasma. The plasma density and parallel wave number obey a fixed relationship for a pure helicon wave propagating in a region of a given magnetic field strength ${ }^{31}$

$$
k=\sqrt{k_{\perp}^{2}+k_{\|}^{2}} \approx \frac{\omega}{k_{\|}} \cdot \frac{\omega_{p}^{2}}{\omega_{c e} c^{2}}=e \mu_{0} v_{p}\left(\frac{n}{B}\right)
$$


where, $k$ is the wave number of the helicon wave, $\mu_{0}$ is the magnetic permeability in vacuum and $v_{p}=\omega / k_{\|}$is the helicon wave phase velocity along the tube. Typically, $k_{\perp}$ is fixed by the tube radius $a$, such that $J_{1}\left(k_{\perp} a\right)=0$, where $J_{1}$ is the Bessel function of the first kind.

Efficient helicon source operation has several desirable characteristics for industry applications and scientific inquiry. These characteristics include very high plasma densities at relatively low temperature for a given rf input power. Consequently, the physical process responsible for efficient helicon source operation has been extensively studied over a wide variety of operating regimes. Possible explanations for this high efficiency includes collisional processes, ${ }^{32}, 33$ Landau damping, ${ }^{34}, 35$ helicon wave penetration, ${ }^{36}$ antenna localized acceleration, ${ }^{37,38}$ mode conversion near the lower hybrid frequency, ${ }^{39}$ and nonlinear trapping of fast electrons. ${ }^{40,41}$ An active area of research for helicon plasma sources focuses on both strong wave damping and high efficiency operation, neither of which is explainable by either Landau damping or collisional processes. Fast electrons are also being studied because they may play a critical role in ionizing the background gas in a helicon source. Fast electrons would appear as a non-Maxwellian component of the electron distribution function.

The mechanism responsible for efficient plasma creation and loss in helicon sources is not completely understood, even in the case of a uniform magnetic field. Other parameters, such as neutral pressure, antenna design, and magnetic field strength, can influence the axial plasma density profile downstream of the antenna. This work focuses on the case of non-uniform magnetic fields, specifically expanding magnetic field geometry, where helicon sources have played a key role in recent studies of spontaneously forming double layers. 


\subsection{HELIX-LEIA}

The helicon plasma source used for these experiments consists of two regions: the small diameter Hot hELIcon eXperiment, (HELIX) where the plasma is produced and the large expansion region known as the Large Experiment on Instabilities and Anisotropies (LEIA). The plasma created in HELIX flows into LEIA (Fig. 2.1). The magnetic field in LEIA is weaker than in HELIX, so the plasma expands into LEIA along divergent magnetic field lines. LEIA has a high beta plasma where "beta" is the ratio of particle pressure to magnetic field pressure, i.e., $\beta=n k_{B} T \mu_{0} / B^{2}$ with $k_{B}$ the Boltzmann constant. The $\beta_{\text {ion }}$ for LEIA and HELIX are $\sim .2$ and $\sim 4$ $\mathrm{x} 10^{-4}$, respectively. The high beta nature of the LEIA plasma makes it ideally suited for laboratory investigations of both heliospheric and magnetospheric physics. The magnetic field expansion geometry in the region between HELIX and LEIA also enables studies of spontaneous, current-free, electrostatic double layer formation at low neutral pressures. Detailed descriptions of the early development of HELIX can be found in the dissertations of Keiter, ${ }^{42}$ Balkey ${ }^{43}$ and Kline. ${ }^{44}$ More recent detailed reports of modifications to the HELIX-LEIA experiment and measurements of the source parameters are found in the dissertations of Sun, ${ }^{45}$ Keesee, ${ }^{46}$ Hardin, ${ }^{47}$ Biloiu ${ }^{48}$ and Chakraborty Thakur. ${ }^{49}$ 


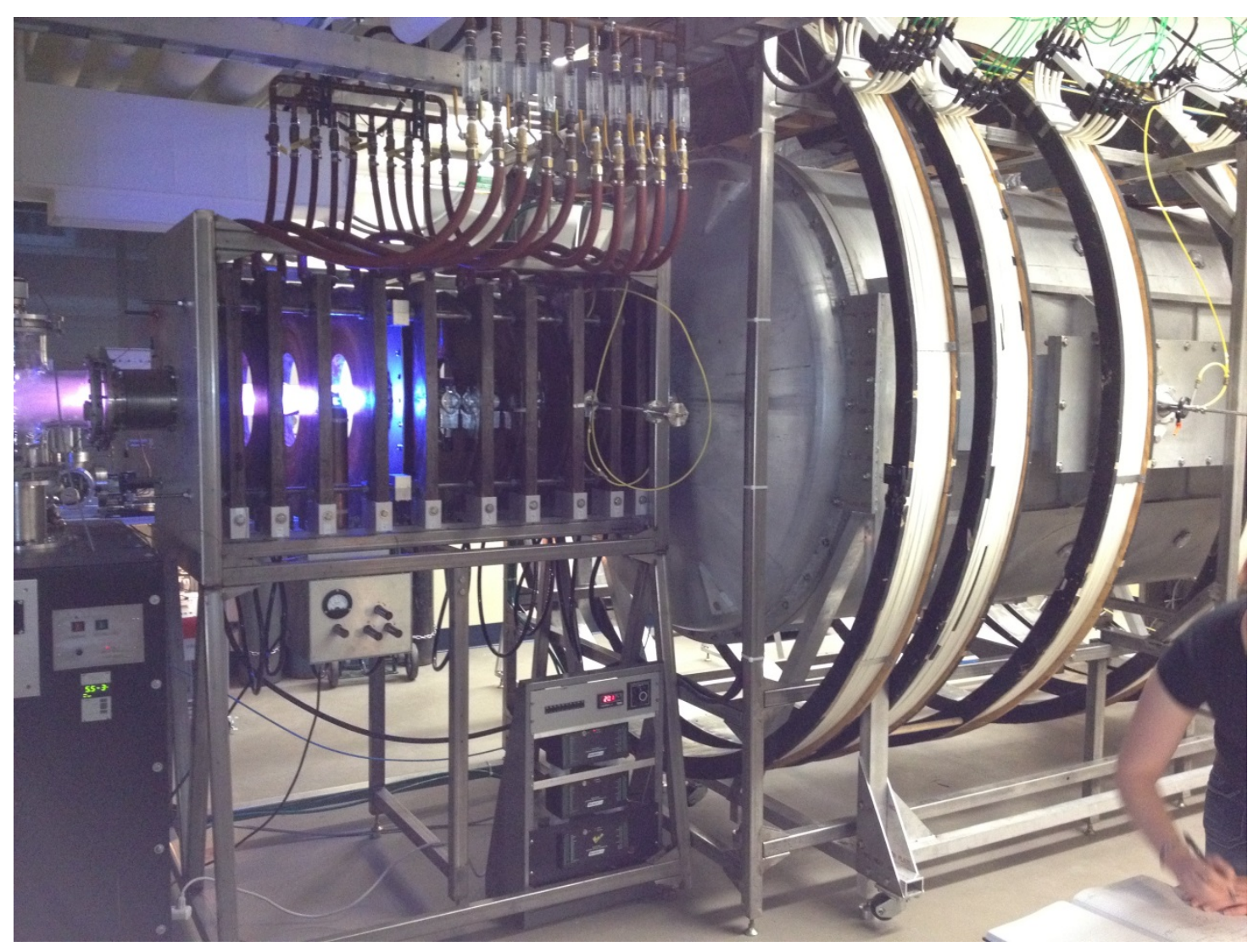

Figure 2.1. HELIX (foreground) and LEIA (large aluminum chamber). HELIX resides inside a Faraday cage, not show here for photography purposes, to provide rf shielding. The large electromagnets surrounding LEIA are approximately $3 \mathrm{~m}$ in diameter.

\subsubsection{Plasma Chamber}

A schematic of the HELIX-LEIA system is shown in Fig. 2.2. The HELIX vacuum chamber is comprised of a $61 \mathrm{~cm}$ long, $10 \mathrm{~cm}$ diameter Pyrex ${ }^{\mathrm{TM}}$ tube connected to a $91 \mathrm{~cm}$ long, $15 \mathrm{~cm}$ diameter stainless steel chamber. The stainless steel chamber opens into a $1.8 \mathrm{~m}$ inner diameter, 2 m outer diameter, $4.5 \mathrm{~m}$ long expansion chamber. The stainless steel chamber has one set of four 6 inch Conflat ${ }^{\mathrm{TM}}$ crossing ports in the center of the chamber and four sets of four 
2.75 inch Conflat ${ }^{\mathrm{TM}}$ crossing ports. The 2.75 inch Conflat $^{\mathrm{TM}}$ crossing ports are spaced evenly on either side of the set of the 6 inch Conflat ${ }^{\mathrm{TM}}$ crossing ports. There are two turbo molecular pumps located at the end of LEIA not attached to HELIX. The other end of the HELIX chamber not attached to LEIA is connected to a glass cross. The three legs of the glass cross are terminated with another turbo-molecular drag pump, an ion gauge, and a 12 inch stainless steel flange fitted with a 4 inch viewport to allow optical access of the plasma along the HELIX-LEIA axis of symmetry. LEIA has several ports for access with scanning internal probes, the reentrant probe, and other diagnostics. 


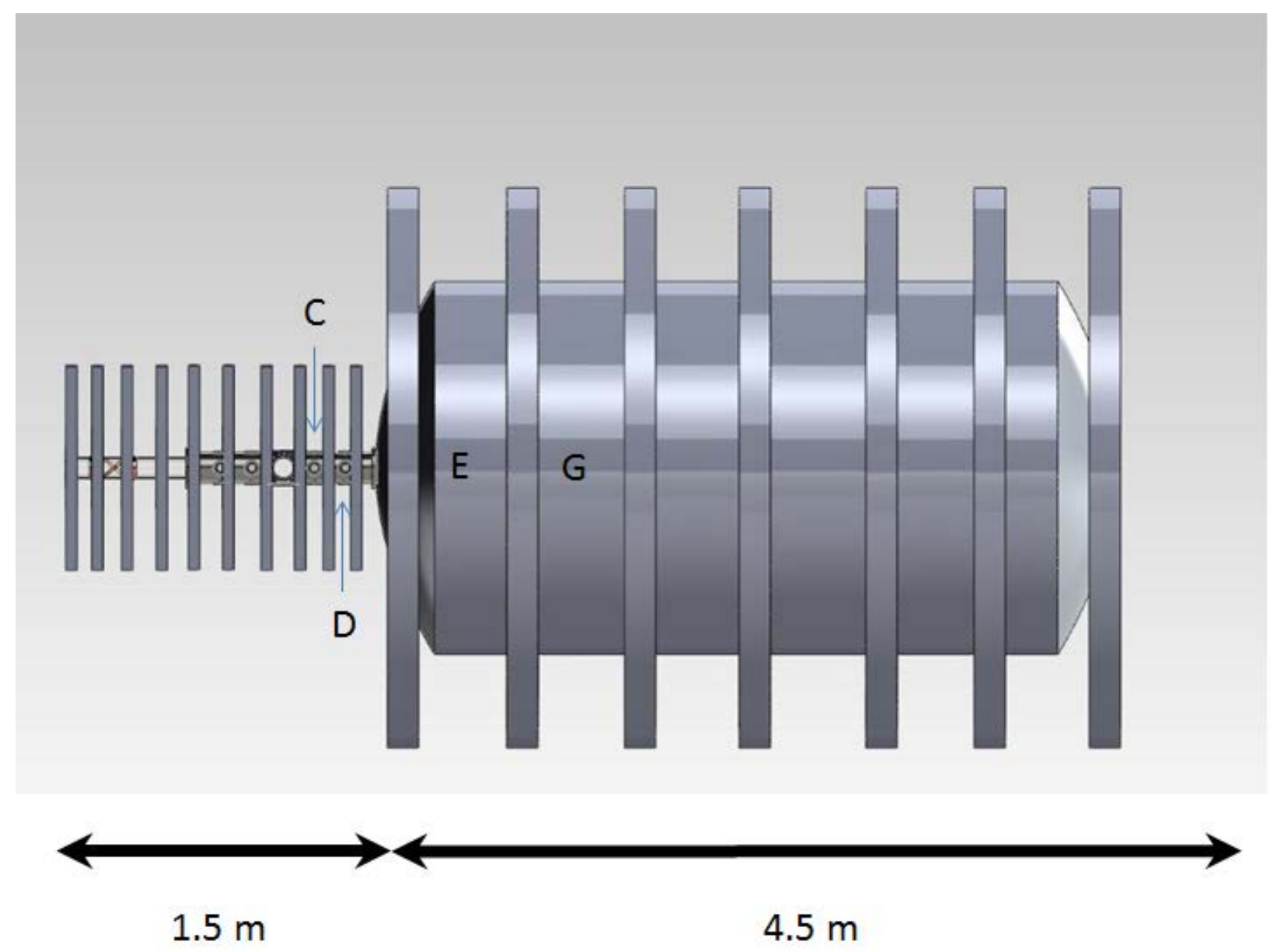

Figure 2.2. Schematic (side view) of the HELIX-LEIA plasma chamber along with labels corresponding to the locations of various diagnostics such as the Langmuir and electrostatic probe(C), RFEA (G) and collection for LIF (G through E).More details in Chapter 3.

\subsubsection{Vacuum System}

Three turbo-molecular drag pumps maintain the vacuum pressure in the chambers. Each of the pumps is backed by its own diaphragm roughing pump. All three turbo pumps are separated from the vacuum chamber by gate valves. The gate valves are on an interlock system designed to close if the pressure rises beyond a threshold value. While the HELIX turbo pump is 
maintained at a constant rotational frequency of $549 \mathrm{~Hz}$, the other two turbo pumps are run at rotational frequencies of $400 \mathrm{~Hz}$ or $600 \mathrm{~Hz}$. There is a slight pressure gradient along the HELIX chamber because of the pressure differential, while the pressure in LEIA is nearly constant. For the current free double layer studies, the gate valve for the HELIX turbo remained shut allowing the neutral pressure to increase in the source region enough to maintain a stable plasma. The two LEIA turbos were operated on the $600 \mathrm{~Hz}$ setting in order to reduce ion-electron and ion-neutral collisions that quench the metastable states needed for laser induced fluorescence. ${ }^{50,51}$ Without gas flowing into the system, the three turbo pumps maintain a base pressure of $10^{-7}$ Torr.

The neutral gas pressure is measured with a series of pressure gauges. A Balzers PKR250 full range pressure gauge is located at one branch of the glass cross and is used by the HELIX pumping station. Another Balzers PKR250 full range pressure gauge is located on the LEIA pumping station. These Balzers gauges have the ability to measure a full range of pressures by combining a cold cathode gauge for pressures below $10^{-2}$ Torr and a Pirani gauge for pressures above $10^{-2}$ Torr. The Balzers pressure gauges require a correction depending on which gas species is used. A Baratron ${ }^{\circledR}$ capacitance manometer pressure gauge is located at approximately the middle of HELIX, $35 \mathrm{~cm}$ downstream of the antenna. The Baratron ${ }^{\circledR}$ gauge pressure measurement is independent of the gas species.

Two MKS1179 mass flow valves regulate the gas flow rate into the system. Typical flow rates while using only one valve are 2.5-6.5 SCCM (standard cubic centimeters per minute) for the CFDL studies in this work. The two flow valves allow for the possibility of regulating a mixture of gases and both flow valves are controlled by a single PR-4000 power supply. There are two gas inlet locations in HELIX. One location is adjacent to the Balzers pressure gauge on 
the glass cross. This inlet location is referred to as the "end feed.” The second inlet location is at a 2.75" cross port on the stainless steel portion of the HELIX chamber closest to the antenna. This inlet location is referred to as the "middle feed" and it allows for a more direct gas flow into the plasma chamber near the antenna instead of relying on diffusion overcoming the pumping at the end gas feed location. All experiments presented here used argon gas introduced with the middle feed. Plasmas are created at neutral pressures (with rf on) ranging from 0.1 to 100 mTorr.

\subsubsection{Magnetic Field}

Ten HELIX water-cooled electromagnets produce an almost uniform, steady state, axial magnetic field of 0-1300 Gauss in the source. These HELIX magnets were donated to WVU by the Max Planck Institüt in Garching, Germany. Each of the 10 magnets has 46 internal copper windings with a resistance of $17 \mathrm{~m} \Omega$ and an inductance of $1.2 \mathrm{mH}$. Two Xantrex XFR power supplies, connected in parallel, provide a total current of up to 400 Amperes to the HELIX magnets. The magnets rest on an adjustable rail system that allows adjustments in axial position and are arranged in an orientation that keeps the field uniform throughout the HELIX magnet array. For the results in sections 5.1 and 5.2, the magnets were water-cooled by building water. For the results in section 5.3, the HELIX magnets were chilled by a Neslab System III Heat Exchanger.

Seven LEIA water-cooled electromagnets produce a steady state axial magnetic field of 0-150 Gauss in the expansion chamber. These LEIA 9' diameter magnets were custom built with each magnet containing five sets of aluminum tubing wound into five two-coil "pancakes" of four layers each, for a total of 40 turns per magnet. The aluminum tubing has 0.5 ” x 0.5 ” square 
cross section, hollowed out to allow for water cooling, and wrapped in insulating paper. For the results in sections 5.1 and 5.2, the magnets were water-cooled by a closed loop system maintained with a Neslab NX-300 chiller. For the results in section 5.3, the LEIA magnets were chilled by building water. The LEIA magnets are powered with a DC EMHP power supply capable of delivering up to 200 Amperes, resulting in a magnetic field of 0-130 Gauss.

Figure 2.3 shows the HELIX-LEIA system drawn to scale (Fig. 2.3 a) with experimental measurements and numerical calculations of the magnetic field profile. Fig. 2.3b shows an axial field gradient of 10 Gauss/cm over a distance of $70 \mathrm{~cm}$ in between HELIX and LEIA. Fig. 2.3c shows the on-axis magnetic field strength and its gradient in the HELIX-LEIA combined system as calculated with a two dimensional numerical model. This simulation was validated with point measurements along the system axis. The simulation was performed assuming a 600 Gauss field in HELIX and two different LEIA fields, 14 Gauss (solid line) and 70 Gauss (dash-dot line). The simulation plot also shows the spatial variation of the contour lines of constant magnetic flux (flux tubes). 

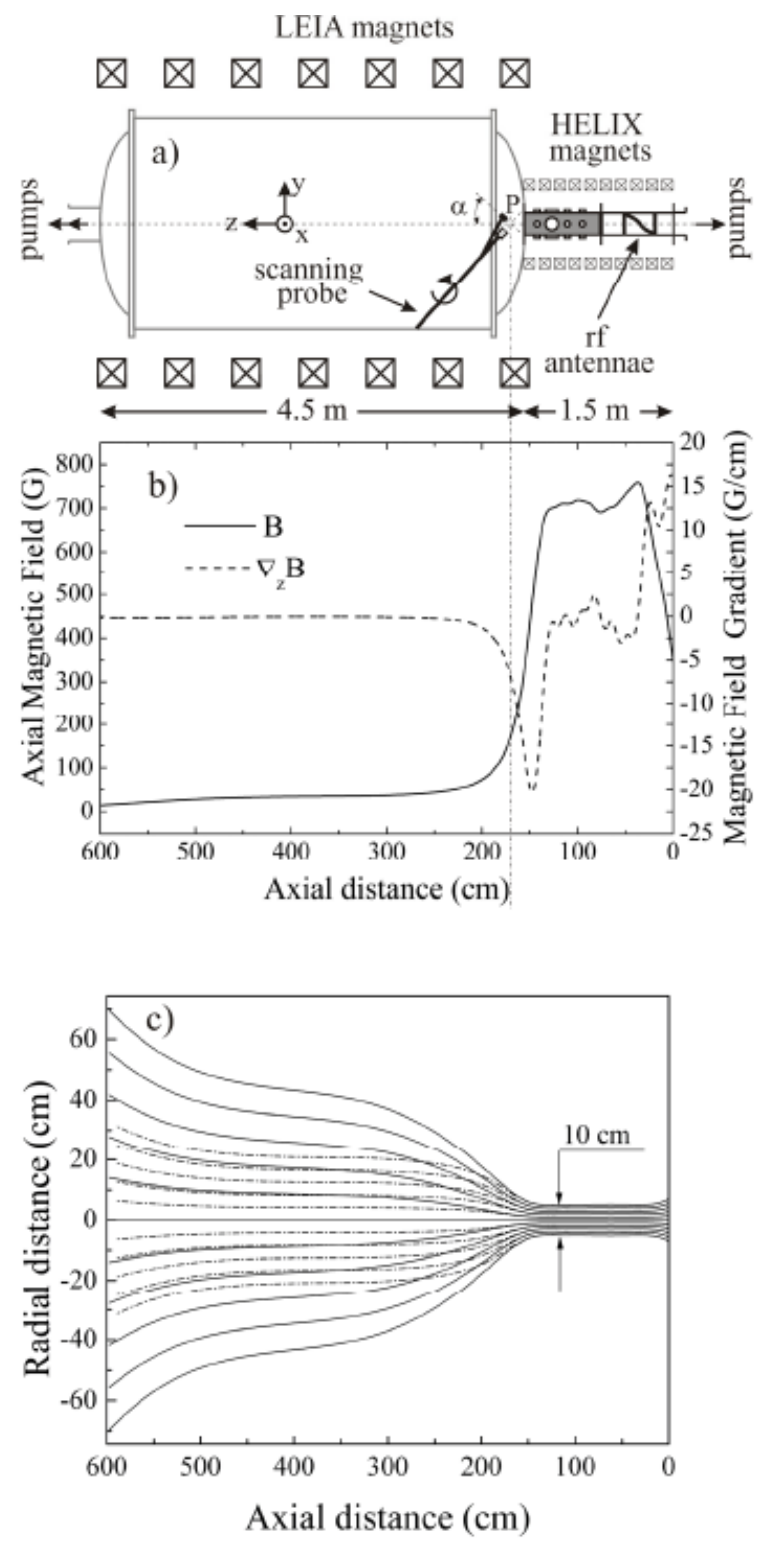

Figure 2.3. (a) HELIX-LEIA device drawn to scale with the magnetic field profiles shown. (b) Magnetic field strength and magnetic field gradient versus axial position over the entire length of the HELIX-LEIA device. (c) Contours of constant magnetic field flux showing the increased divergence that results when the magnetic field in the LEIA decreases from $70 \mathrm{G}$ (dash-dot line) to $14 \mathrm{G}$ (solid line), for a constant $600 \mathrm{G}$ magnetic field in HELIX. Figure is adapted from Ref. [47]. 


\subsubsection{Rf Antenna and Matching Network}

The antenna, shown in Fig. 2.4, is tightly wrapped around the Pyrex tube at $37 \mathrm{~cm}$ from the end of the source chamber. Rf power of up to $2.0 \mathrm{~kW}$ over a frequency range of $6-18 \mathrm{MHz}$ is coupled into a $19 \mathrm{~cm}$ half wave, helical antenna to create the steady state plasma. Additional details of the antenna and matching network can be found in the dissertations of Balkey ${ }^{52}$ and Sun. ${ }^{53}$ The rf system is comprised of a $50 \mathrm{MHz}$ Wavetek model-80 function generator that supplies a rf signal to a $30 \mathrm{~dB}$ ENI 1000 amplifier. The amplifier delivers up to $2 \mathrm{~kW}$ of power within the frequency range of 6-18 MHz. A $\pi$ matching network is connected to the amplifier with a high frequency co-axial cable. The purpose of the matching network is to match the $50 \Omega$ output impedance of the amplifier to the antenna/matching network system. The matching network consists of three tuning capacitors and a load capacitor. All of the capacitors are high voltage Jennings vacuum capacitors. Two of the tuning capacitors have a range of 4- $250 \mathrm{pF}$. The remaining tuning capacitor has a range of 5-500 pF. The large load capacitor has a range of 20$2000 \mathrm{pF}$. The three tuning capacitors are connected in parallel to each other and in series with the antenna. The load capacitor is in parallel with the tuning capacitors and the antenna. The capacitors are connected to the antenna with silver-plated copper rods. Fig. 2.5 shows the antenna/matching network system. The real impedance of the matching network must equal the $50 \Omega$ output impedance of the amplifier while the imaginary part of the combined matching network-antenna circuit must be zero to maximize the forward power coupled to the antenna and to minimize power reflected back to the amplifier. For a $\pi$ matching network, Chen calculated the required capacitances for the load and tuning capacitors to be 


$$
C_{L}=\frac{1}{2 \omega R}\left[1-\left(1-\frac{2 R}{R_{0}}\right)\right]^{1 / 2}
$$

and

$$
C_{T}=\left[\omega X-\frac{1-R / R_{0}}{C_{L}}\right]^{-1}
$$

where $R$ is the real resistance of the antenna, and $X=\omega L$ is the reactive impedance of the antenna.
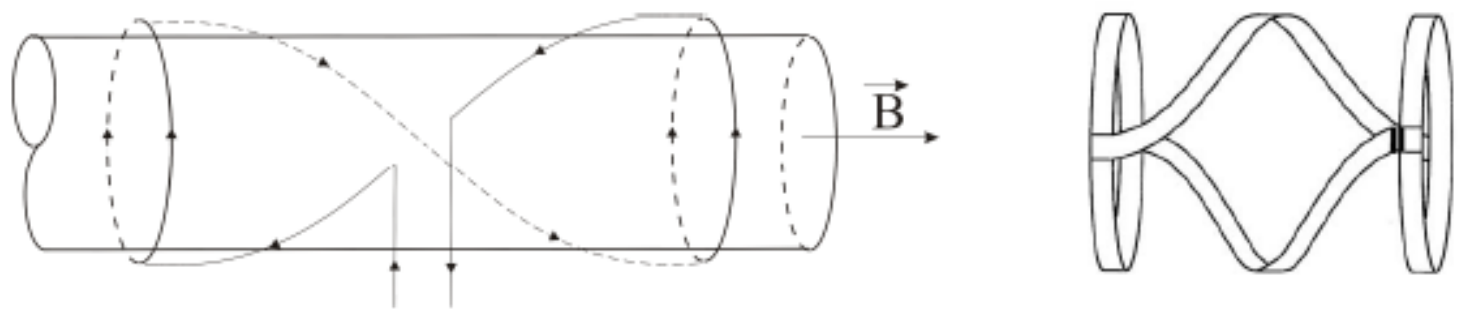

Figure 2.4. Diagram of a $m=+1$ helical antenna and the actual design of the antenna. ${ }^{61}$ Figure is adapted from Ref. [47]. 


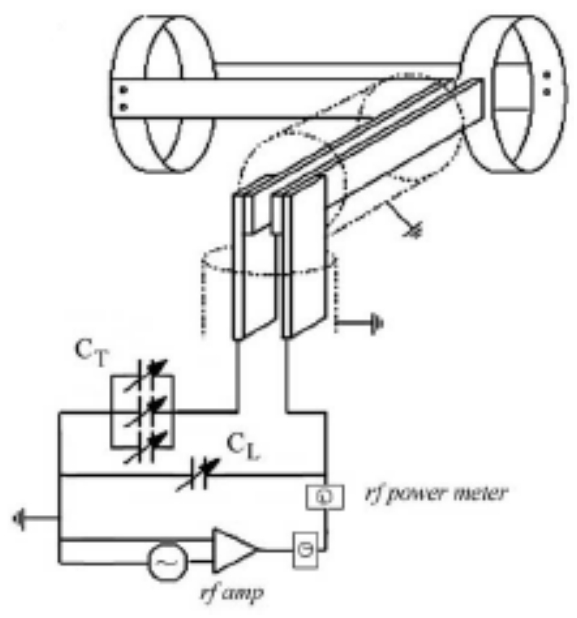

Figure 2.5. Matching circuit for the helicon antenna for HELIX. $C_{L}$ is the load capacitor and $C_{T}$ denotes the three tuning capacitors. Figure is adapted from Ref. [47].

Once the discharge is initiated, the effect of the inductive load of the plasma on the antenna has to be considered. For a typical helicon plasma source in the "helicon" or "inductive" mode, Eq. 2.3 becomes

$$
C_{T}^{-1}=\omega^{2} L-\frac{\left(1-\frac{R}{R_{0}}\right)}{C_{L}}
$$

where $L$ is the total inductance in the antenna portion of the matching network-antenna circuit. 


\subsubsection{HELIX-LEIA Plasma Parameters}

Characteristic electron temperature and density in HELIX for these experimental parameters are $T_{e}=5.8 \mathrm{eV}$ and $n=2 \times 10^{12} \mathrm{~cm}^{-3}$ as measured with rf compensated cylindrical Langmuir probes located $50 \mathrm{~cm}$ downstream of the antenna.

\begin{tabular}{|c|c|c|}
\hline Parameter (units) & HELIX & LEIA \\
\hline$B$ (Gauss) & $500-1200$ & $5-70$ \\
\hline$p_{\text {neutral }}$ (mTorr) & $1.5-10$ & $0.15-1$ \\
\hline$n\left(\mathrm{x} 10^{12} \mathrm{~cm}^{-3}\right)$ & $0.1-10$ & $0.001-0.1$ \\
\hline$T_{e}(\mathrm{eV})$ & $4-12$ & $2-8$ \\
\hline$T_{i}(\mathrm{eV})$ & $<1$ & $<1$ \\
\hline$\lambda_{\text {Debye }}(\mathrm{cm})$ & $5 \times 10^{-3}-8 \times 10^{-4}$ & $3 \times 10^{-2}-6.5 \times 10^{-2}$ \\
\hline$r_{e}(\mathrm{~cm})$ & $4 \times 10^{-2}-2 \times 10^{-2}$ & $5 \times 10^{-2}-1.5$ \\
\hline$r_{i}(\mathrm{~cm})$ & $3 \times 10^{-1}-6 \times 10^{-1}$ & $4-60$ \\
\hline$\omega_{p e}\left(10^{10} \mathrm{rad} / \mathrm{s}\right)$ & $1.8-18$ & $0.18-18$ \\
\hline$\omega_{c e}\left(10^{9} \mathrm{rad} / \mathrm{s}\right)$ & $5.2-34$ & $52-340$ \\
\hline$\omega_{c i}\left(10^{6} \mathrm{rad} / \mathrm{s}\right)$ & $0.12-0.3$ & $1.2 \times 10^{-3}-1.6 \times 10^{-2}$ \\
\hline
\end{tabular}




\section{Chapter 2 References}

${ }^{1}$ E.E. Scime, A.M. Keesee, and R.W. Boswell, Phys. Plasmas, 15, 058301 (2008).

${ }^{2}$ J. P. Squire, F. R. Chang-Diaz, T. W. Glover, V. T. Jacobson, D. G. Chavers, R. D. Bengtson, E. A. Bering III, R. W. Boswell, R. H. Goulding and M. Light, Fusion Sci. Technol. 43, 111 (2003).

${ }^{3}$ R. W. Boswell, O. Sutherland, C. Charles, J. P. Squire, F. R. Chang-Diaz, T. W. Glover, V. T. Jacobson, D. G. Chavers, R. D. Bengtson, E. A. Bering, R. H. Goulding and M. Light, Phys. Plasmas 11, 5125 (2004).

${ }^{4}$ X. Sun, C. Biloiu, R. Hardin and E. E. Scime, Plasma Sources Sci. Technol. 13, 359 (2004).

${ }^{5}$ R. Winglee, T. Ziemba, L. Giersch, J. Prager, J. Carscadden and B. R. Roberson, Phys. Plasmas 14, 63501 (2007).

${ }^{6}$ G. W. Gibson Jr. and D. J Hemker, Semiconductor International 19, 193 (1996).

${ }^{7}$ S. Kimura and H. Ikoma, J. Appl. Phys. 85, 551 (1999).

${ }^{8}$ E. E. Scime, P. A. Keiter, M. M. Balkey, R. F. Boivin, J. L. Kline, M. Blackburn and S. P. Gary, Phys. Plasmas 7, 2157 (2000).

${ }^{9}$ J. Hanna and C. Watts, Phys. Plasmas 8, 4251 (2001).

${ }^{10}$ K. Itoh, S. Itoh, P. H. Diamond, T. S. Hahm, A. Fujisawa, G. R. Tynan, M. Yagi and Y. Nagashima, Phys. Plasmas 13, 55502-1 (2006).

${ }^{11}$ M. I. Panevsky and R. D. Bengston, Phys. Plasmas 11, 4196 (2004).

${ }^{12}$ G. N. Harding and P. C. Thonemann, Proc. Phys. Soc. 85, 317 (1965).

${ }^{13}$ R. Bowers, C. Legendy and F. E. Rose, Phys. Rev. Lett. 7, 339 (1961).

${ }^{14}$ L. C. Woods, J. Fluid Mech. 18, 401 (1964). 
${ }^{15}$ J. P. Klozenberg, B. McNamara and P. C. Thonemann, J. Fluid Mech. 21, 545 (1965).

${ }^{16}$ B. J. Davies and P. J. Christiansen, Plasma Phys. 11, 987 (1969).

${ }^{17}$ R. W. Boswell, Plasma Phys. Controlled Fusion 26, 1147 (1970).

${ }^{18}$ R. W. Boswell, A Study of Wave in Gaseous Plasma, Ph. D. Thesis, Flinders University, Adelaida, Australia (1974).

${ }^{19}$ R. W. Boswell, R. K. Porteus, A. Bouchoule and P. Ranson, Phys. Lett. A 91, 163 (1982).

${ }^{20}$ R. W. Boswell and D. Henry, Appl. Phys. Lett. 47, 1095 (1985).

${ }^{21}$ R. W. Boswell and R. K. Porteus, J. Appl. Physics. 62, 3123 (1987).

${ }^{22}$ K. Takahashi, T. Lafleur, C. Charles, P. Alexander, and R. W. Boswell, Phys. Rev. Lett. 107, 235001 (2011).

${ }^{23}$ C. Charles, R. W. Boswell and K. Takahashi, Plasma. Phys Control. Fusion 54 (2012) 124021.

${ }^{24}$ R. W. Boswell and F. F. Chen, IEEE Trans. Plasma Sci. 25, 1229 (1997).

${ }^{25}$ F. F. Chen and R. W. Boswell, IEEE Trans. Plasma Sci. 25, 1245 (1997).

${ }^{26}$ E. E. Scime, A. M. Keesee and R. W. Boswell, Phys. Plasmas 15, 058301 (2008).

${ }^{27}$ R. W. Boswell, A Study of Wave in Gaseous Plasma, Ph. D. Thesis, Flinders University, Adelaida, Australia (1974).

${ }^{28}$ Barkhausen, 1919. Physik Zeit., 20, 401.

${ }^{29}$ Storey, L. R. O., An investigation of whistling atmospherics, Phil. Trans. Roy. Soc., 246, 113141, 1953.

${ }^{30}$ P. Aigrain, Proc. Int. Conf. Semiconductor Physics, 224 (1960).

${ }^{31}$ F. F. Chen, Plasma. Phys. Controlled Fusion 33, 339 (1991).

${ }^{32}$ R. W. Boswell and F. F. Chen, IEEE Trans. Plasma Sci. 25, 1229 (1997). 
${ }^{33}$ F. F. Chen and R. W. Boswell, IEEE Trans. Plasma Sci. 25, 1245 (1997).

${ }^{34}$ Y. Mouzouris and J. E. Scharer, Phys. Plasmas 5, 4253 (1998).

${ }^{35}$ F. F. Chen and D. D. Blackwell, Phys. Rev. Lett. 82, 2677 (1999).

${ }^{36}$ R. Kinder and M. J. Kushner, J. Vac. Sci. Technol. A 19, 76 (2001).

${ }^{37}$ G. G. Borg, J. Bright and I. V. Kamenski, Plasma Phys. Controlled Fusion 40, 987 (1998).

${ }^{38}$ A. W. Degeling and R. W. Boswell, Phys. Plasmas 4, 2748 (1997).

${ }^{39}$ S. Cho and J. G. Kwak, Phys. Plasmas 4, 4167 (1997).

${ }^{40}$ R. T. S. Chen and N. Hershkowitz, Phys. Rev. Lett. 80, 4677 (1998).

${ }^{41}$ A. W. Degeling, N. Mikhelson, R. W. Boswell and N. Sadeghi, Phys. Plasmas 5, 572 (1998).

${ }^{42}$ P.A. Keiter, Experimental Investigation of Ion Temperature Anisotropy Driven Instabilities in a High Beta Plasma, Ph. D. Thesis, West Virginia University, Morgantown (1999).

${ }^{43}$ M. M. Balkey, Optimization of a Helicon Plasma Source for Maximum Density with Minimal Ion Heating, Ph. D. Thesis, West Virginia University, Morgantown (2000).

${ }^{44}$ J. L. Kline, Slow Wave Ion Heating and Parametric Instabilities in the HELIX Helicon Source, Ph. D. Thesis, West Virginia University, Morgantown (2002).

${ }^{45}$ X. Sun, A Study of Ion Acceleration, Asymmetric Optical Pumping and Low Frequency Waves in Two Expanding Helicon Plasmas, Ph. D. Thesis, West Virginia University, Morgantown (2005).

${ }^{46}$ A. M. Keesee, Neutral Density Profiles in Argon Helicon Plasmas, Ph. D. Thesis, West Virginia University, Morgantown (2005).

${ }^{47}$ R. A. Hardin, Measurement of Short-Wavelength Electrostatic Fluctuations in a Helicon Plasma Source, Ph. D. Thesis, West Virginia University, Morgantown (2008). 
${ }^{48}$ I. A. Biloiu, Laser Induced Fluorescence Studies of Ion Acceleration in Single and Multiple Species Expanding Plasmas, Ph. D. Thesis, West Virginia University, Morgantown (2009).

${ }^{49}$ S. Chakraborty Thakur, Understanding Plasmas through Ion Velocity Distribution Function measurements, Ph. D. Thesis, West Virginia University, Morgantown (2010).

${ }^{50}$ S. A. Cohen, N. S. Seifert, S. Stange, E. E. Scime, R. F. Boivin and F. Levinton, Phys. Plasmas 10, 2593 (2003).

${ }^{51}$ A.M. Keesee, E. E. Scime, C. Charles, A. Meige and R. W. Boswell Phys. Plasmas 12, $093502(2005)$.

${ }^{52}$ M. M. Balkey, Optimization of a Helicon Plasma Source for Maximum Density with Minimal Ion Heating, Ph. D. Thesis, West Virginia University, Morgantown (2000).

${ }^{53}$ X. Sun, A Study of Ion Acceleration, Asymmetric Optical Pumping and Low Frequency Waves in Two Expanding Helicon Plasmas, Ph. D. Thesis, West Virginia University, Morgantown (2005). 


\section{Chapter 3: Standard Plasma Diagnostics}

Standard plasma diagnostics can be divided in to three broad categories: ex situ, in situ intrusive and in situ nonintrusive. Ex situ measurements are typically made with samples of plasma reactor contents, where the contents are removed from the system and studied in a different environment. Technically, all in situ measurements are intrusive. However the intrusive effects can be considered negligible in some cases compared to the overall system behavior; this is the case that is denoted as "in situ nonintrusive." The term "in situ intrusive" is reserved for cases where the perturbation introduced by the measurement is not negligible and must be considered during analysis. All measurements of the HELIX-LEIA plasma in this work are in situ.

The five diagnostics used in this work are Langmuir probes, electrostatic probes, retarding field energy analyzers (RFEAs), continuous wave laser induced florescence (CW LIF), and time-resolved LIF. Langmuir probes are an example of an in situ intrusive measurement. A Langmuir probe is simply a conductor that is inserted into the plasma and the current drawn by the probe is measured as a function of varying bias voltage. The probe perturbs the plasma, but the perturbation is often negligible if the probe size and bias voltages are carefully chosen. A RFEA probe is typically a much larger obstruction than a Langmuir probe and its perturbative effects cannot be ignored during data analysis. LIF (CW or time-resolved) is an in situ nonintrusive diagnostic as long as the injection of the laser light source and the emitted fluorescence light is collected outside of the vacuum chamber. These are well established diagnostic techniques that have been applied to the HELIX-LEIA apparatus with specific modifications for the experiments described in this work. 


\subsection{Langmuir Probe}

Irving Langmuir is credited with the first technical description and the theoretical framework for this type of electrostatic probe. ${ }^{1}$ Consequently, it now carries the name “Langmuir probe.” From measurements drawn by a Langmuir probe, the electron temperature and density of a plasma is determined. Analysis of Langmuir probe can prove to be challenging, especially in cases of drifting, non-Maxwellian, or collisional plasmas, all which require comparison of the measurements with complex theoretical models. Thorough reviews on Langmuir probe theory and operation can be found in articles by Chen, ${ }^{2}$ Schott, ${ }^{3}$ Hutchinson, ${ }^{4}$ Hershkowitz, ${ }^{5}$ and Demidov et al., ${ }^{6}$ Sheridan et al., ${ }^{7}$ and others. The subsections that follow will give a brief overview of the theory, design, and data analysis relevant to the probes used in these experiments.

\subsubsection{Langmuir Probe Theory}

By sweeping through different bias voltages and collecting the current drawn by a Langmuir probe immersed in a plasma, an I-V trace, also known as an I-V characteristic, is obtained. Fig. 3.1 is diagram of an ideal I-V trace. ${ }^{8}$ 


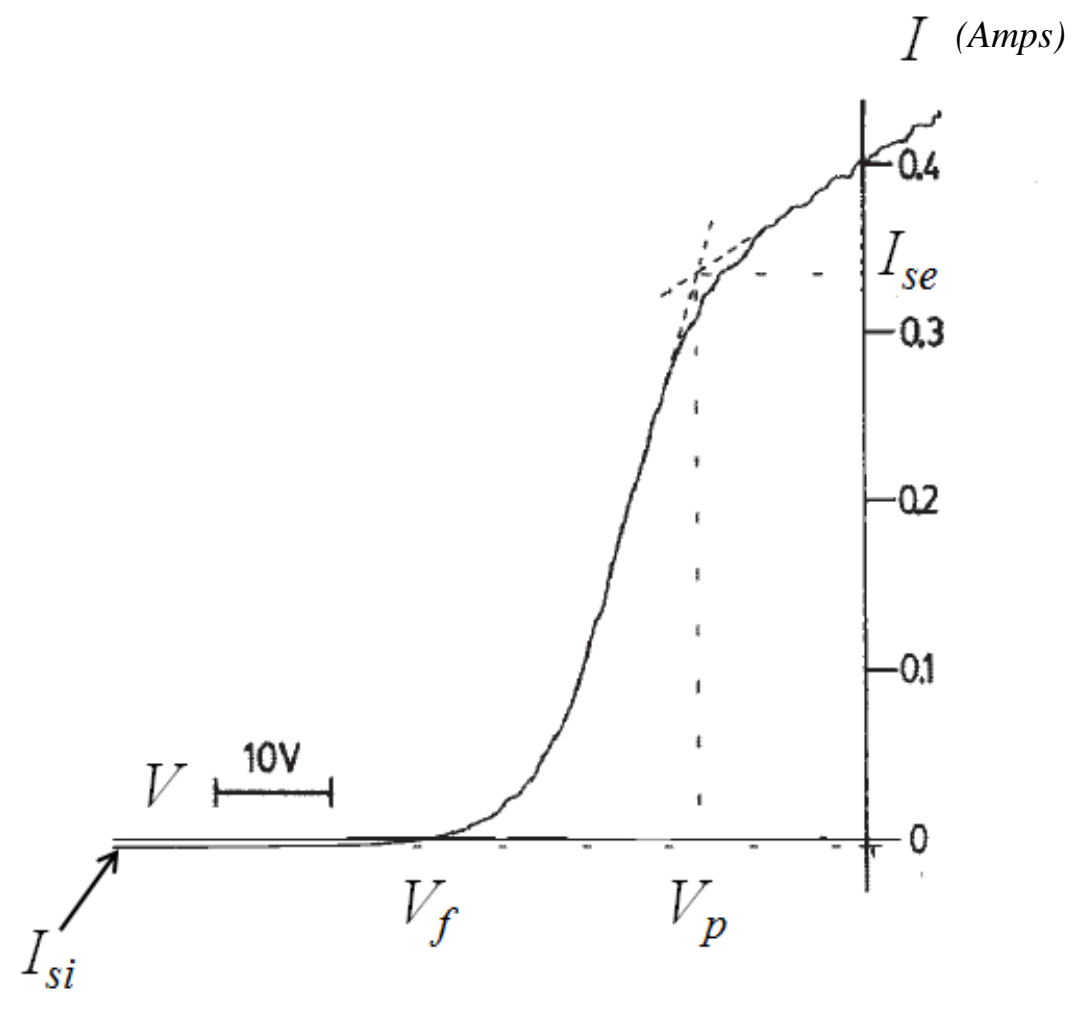

Figure 3.1. Idealized Langmuir probe I-V characteristic. Figure obtained from Ref. [4]

Quasi-neutral plasmas typically cause the probe to charge negatively because the electrons have a higher flux than the ions due to their lighter mass and higher mean velocities. The floating potential, labeled $V_{f}$ on the horizontal axis of Fig 3.1, is the voltage to which the probe charges such that the current drawn by the probe vanishes. The floating potential is not the electric potential of the plasma. This latter potential is called the plasma or space potential, $V_{p}$. If the probe is at the plasma potential, the collected current is nearly the maximum electron current possible. Using a high impedance voltage source to control the probe's bias voltage, different regimes are observed. As the applied voltage becomes more negative than the floating potential, the probe repels electrons and attracts more ions; resulting in a negative current value. The ion saturation current regime is achieved by making the applied potential even more negative, to the 
point where all of the electrons are repelled and the ion current reaches an absolute maximum. Varying the applied bias voltage in the opposite direction, more positive than the floating potential, the probe shifts into the electron saturation current regime. The bias voltage at which the slope of the I-V trace begins to decrease near the electron saturation current (the $2^{\text {nd }}$ knee) is the plasma potential. When the applied bias voltage is at the plasma potential, there is no potential difference between the plasma and the probe. Since electrons are more mobile than ions, the magnitude of the electron saturation current is much greater than the ion saturation current.

Further understanding of the different regions of the I-V characteristic clarifies how the shape of the I-V trace is dependent upon the plasma density, the electron temperature and the shape of the probe. Assuming the particle distribution is Maxwellian, the plasma is collisionless, and there is no magnetic field, the current in the region by the $1^{\text {st }}$ knee in Fig 2.1 around the floating potential is approximated by,

$$
\begin{aligned}
I\left(V_{o}-V_{p}\right)= & n_{e} e A_{p}\left(\frac{k_{B} T_{e}}{m_{i}}\right)^{1 / 2}\left[\frac{1}{2}\left(\frac{2 m_{i}}{\pi m_{e}}\right)^{1 / 2} \exp \left(\frac{e\left(V_{o}-V_{p}\right)}{k_{B} T_{e}}\right)\right. \\
& \left.-\frac{A_{s}}{A_{p}} \exp \left(-\frac{1}{2}\right)\right],
\end{aligned}
$$

where $V_{o}$ is the applied voltage, $V_{p}$ is the plasma potential, $n_{e}$ is the plasma density, $e$ is the electronic charge, $T_{e}$ is the electron temperature, $m_{i}$ is the ion mass, $m_{e}$ is the electron mass, $A_{s}$ is the area of the sheath, and $A_{p}$ is the surface area of the probe. ${ }^{4} \mathrm{~A}$ good approximation of the ratio $A_{s} / A_{p}$ is $\approx 1$ if the probe size is much larger than the thickness of the sheath surrounding the probe. The sheath is a region of spatially varying potential, created when the charges in the 
plasma shield the potential applied to the probe. ${ }^{9}$ As $V_{o}$ becomes more negative, the first term in the brackets of Eq. 3.1 is negligible. Keeping the second term in the brackets, Eq. 3.1 is

$$
I_{s i}=-0.61 e n_{e} A_{p} \sqrt{T_{e} / m_{i}}
$$

where $I_{s i}$ and $J_{i}$ are the ion saturation current and the ion current density respectively in an unmagnetized plasma.

Plasma density and the electron temperature are the two unknowns in Eq. 3.1. The electron temperature is obtained by taking the derivative of Eq. 3.1 with respect to the voltage seen by the plasma, $=V_{o}-V_{p}$, giving

$$
\frac{d I(V)}{d V} \cong \frac{e}{T_{e}}\left(I-I_{s i}\right)+\frac{d I_{s i}}{d V}
$$

The second term is dropped because $d I_{s i} / d V \ll d I(V) / d V$ in the saturation regime. Consequently, Eq. 3.3 is rewritten to provide an estimate of electron temperature,

$$
T_{e}=\frac{e\left[I(V)-I_{s i}\right]}{\frac{d I(V)}{d V}} .
$$

The electron temperature is determined by performing a linear fit to the semi-logarithmic based plot of $\ln \left(I-I_{s i}\right)$ versus $V$ and taking the inverse of the slope of the fit. The calculated electron temperature along with measured ion saturation current is then used to solve for the plasma density via Eq. 3.2. 
There are some differences between the ideal I-V trace and an experimentally obtained IV trace for a cylindrical Langmuir probe in high density plasmas such as a helicon source. In the helicon source, a cylindrical Langmuir probe cannot achieve true electron saturation because the sheath surrounding the probe continues to expand and collects more electrons as the voltage is increased. ${ }^{8}$ Without the second knee in the I-V trace, the plasma potential cannot be directly measured either and has to be approximated.

For $T_{i}<T_{e}$, there is a useful relationship between the plasma potential and the floating potential. The ion current at the floating potential is

$$
j_{i}=\frac{1}{4} n e \sqrt{\frac{8 k_{B} T_{e}}{\pi m_{i}}}
$$

and the electron current at the floating potential is

$$
j_{e}=\frac{1}{4} n e \sqrt{\frac{8 k_{B} T_{e}}{\pi m_{e}}} \exp \left(\frac{e\left(V_{f}-V_{p}\right)}{k_{B} T_{e}}\right) .
$$

Since the total current on the probe is zero at the floating potential, Eq. 3.5 is set equal to Eq. 3.6. After some manipulation of terms, the plasma potential is expressed as ${ }^{10}$

$$
V_{p}=V_{f}+\frac{k_{B} T_{e}}{2 e} \ln \left(\frac{T_{e} m_{i}}{T_{e} m_{e}}\right)=V_{f}+\frac{k_{B} T_{e}}{2 e} \ln \left(\frac{m_{i}}{m_{e}}\right)
$$

For argon ions, where $m_{i}=40 m_{p}$ and $m_{p}$ is the mass of a proton, Eq. 3.7 becomes

$$
V_{p}=V_{f}+5.6 T_{e}
$$


This shows that for argon, the difference between the plasma and the floating potentials is approximately six times the electron temperature and that the slope $d I / d V_{\text {applied }}$ can used as reasonable approximation to calculate the electron temperature rather than having to use the slope of $d I / d V$.

Magnetic fields have been ignored up until this point in the discussion of Langmuir probe theory. The introduction of magnetic fields causes ions and electrons to gyrate about the field lines, limiting cross field transport and restricting the flux of particles to the probe. The importance of the magnetic field effects is determined by the ratio of the gyroradius to the characteristic dimension of the probe. If this ratio is much less than unity for a given species, that species will be impeded from interacting with the probe. Eq. 3.1-3.4 must then be modified to account for cross field transport and collisions. ${ }^{4}$ For an ion temperature of $0.3 \mathrm{eV}$ and a magnetic field strength of 1000 Gauss in HELIX, the gyroradius for an ion is $\approx 3.5 \mathrm{~mm}$ which is comparable to the probe tip length of $2 \mathrm{~mm}$ and larger than the tip diameter of $0.5 \mathrm{~mm}$. Including magnetic field effects on the ions, Hutchinson ${ }^{4}$ showed that Eq. 3.2 must be adjusted:

$$
I_{s i}=-0.49 e n_{e} A_{p} \sqrt{T_{e} / m_{i}}
$$

This discussion of Langmuir probe theory has also ignored the effect strong rf fields have on probe measurements. Rf fields in the helicon source cause both acceleration and deceleration of the electrons towards the probe. The sloshing of the electrons back and forth in the sheath introduces error in the Langmuir probe measurements for the floating potential and a broadening of the electron distribution function. ${ }^{11}$ To minimize these effects on measurements, the Langmuir 
probes must be rf compensated. Sudit and Chen ${ }^{12}$ developed an if compensation method involving an addition of a floating electrode. The floating electrode is exposed to the plasma potential fluctuations and connected to a probe tip through a small capacitor, forcing the probe tip to follow the potential oscillations and thereby reduce the sheath impedance.

The Langmuir probe used in these experiments has such an electrode. However, it is not directly exposed to the plasma. A set of rf chokes are also connected in between the probe tip and the voltage source. The chokes increase the impedance of the current measurement circuit at the $r f$ frequency.

\subsubsection{Langmuir Probe Design}

Fig 3.2 shows a schematic of the Langmuir probe used in these experiments. The probe tip is a graphite rod, $0.5 \mathrm{~mm}$ in diameter and available commercially for mechanical pencils. The graphite rods are inserted into $0.6 \mathrm{~mm}$ alumina shaft and attached by a set screw to a copper base. The copper base has a $10 \mathrm{nF}$ capacitor and a chain of rf chokes connected to it. A Boron Nitride (BN) cap with a hole in the center allows the alumina shaft and the exposed graphite tip to extend into the plasma, while the capacitor and rf choke assembly remains protected. The threaded BN cap screws into a threaded stainless steel probe shaft. The rf chokes are 0.25 Watt, shielded, resonant, inductors from Lenox-Fugle International, Inc. Each of the inductors is designed to block a particular range of rf frequencies. 


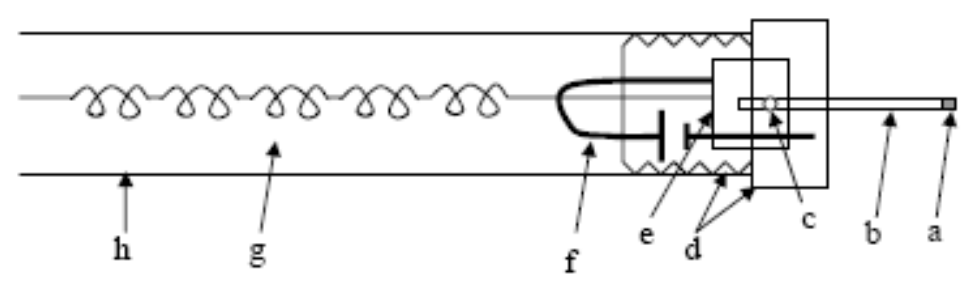

Figure 3.2. The Langmuir probe including a) graphite probe tip, b) alumina shaft, c) set screw, d) threaded boron nitride cap, e) copper base, f) capacitor, g) chain of rf chokes and h) stainless steel probe shaft. Figure obtained from Ref. [13].

From the copper base, the rf chokes are placed in the following order (units of $\mathrm{MHz}$ ): 26, 53, 26, 13.2 and 6.8. The end of the rf chokes is then soldered to a shielded, coaxial probe wire. The probe wire is attached to the BNC vacuum feedthrough at the far end of the probe shaft. Thermaflex tubing is used to cover the chain of rf chokes. ${ }^{13}$ A picture of the Langmuir probe head ${ }^{14}$ used in HELIX is shown in Fig 3.3. To minimize the plasma perturbation, the shaft of the Langmuir probe inserted into the plasma is less than $1 \mathrm{~mm}$ in diameter. 


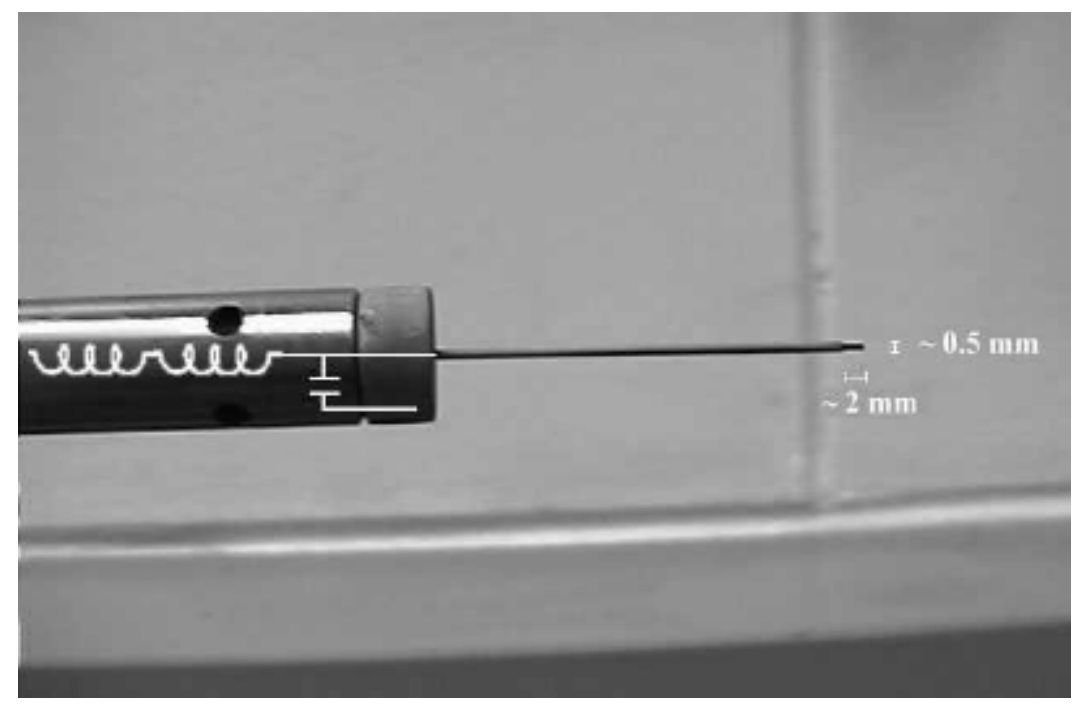

Figure 3.3. The Langmuir probe head. The exposed graphite tip is $2 \mathrm{~mm}$ long and runs the length of the alumina tube into the boron nitride cap. The capacitor and the inductor chains are shown schematically. Figure obtained from Ref. [14].

Varying of a bias voltage and measurement of the corresponding probe currents that build an I-V trace is accomplished with a Keithley 2400 SourceMeter. The source meter is controlled by custom software created in LabWindows ${ }^{\mathrm{TM}}$ via a GPIB interface. Fig 3.4 shows a schematic drawing of the Langmuir probe measurement circuit. In the experiments reported here, the Langmuir probe measurements were obtained $50 \mathrm{~cm}$ downstream from the antenna at location $\mathrm{C}$ shown in Fig. 2.2. 


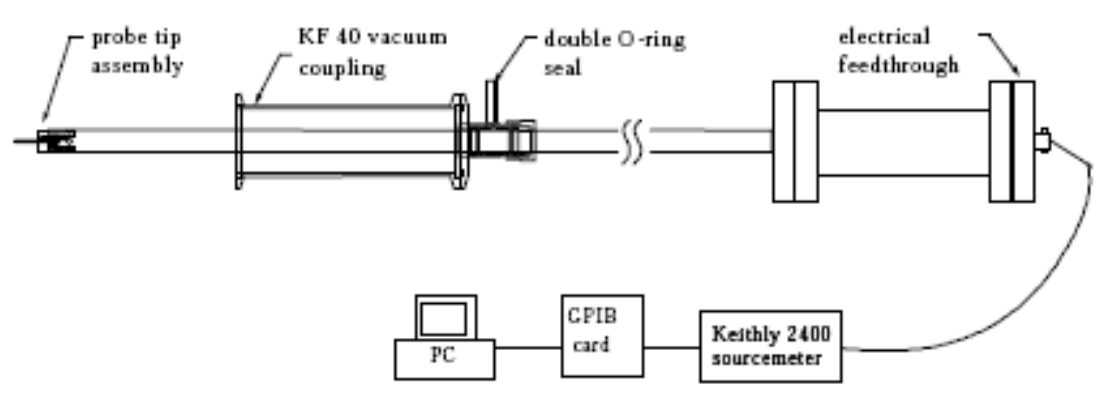

Figure 3.4. The Langmuir probe measurement circuit. Figure obtained from Ref. [14].

\subsection{Electrostatic Probe}

The electrostatic probe used for electrostatic fluctuation measurements is essentially an uncompensated multi-tip Langmuir probe. The spatial separation between the different tips allows for the measurement of differences in floating potential due to a propagating wave. Rf compensation is avoided in these probes to allow for a full range of observable fluctuation frequencies, including up to the rf driving frequency. Observed fluctuations are assumed to be fluctuations in floating potential, not electron temperature. Typically, emissive probes are used to measure electron temperature fluctuations. ${ }^{15}$ However, emissive probes do not work well in steady state helicon plasmas with densities of the order of $10^{13} \mathrm{~cm}^{-3}$.

\subsubsection{Electrostatic Probe Theory}

Without a bias voltage, the conducting tips of the electrostatic probe are allowed to charge up to the floating voltage at which point no current flows to the probe. When the plasma surrounding the probe undergoes a change due to electrostatic wave activity, the particle flux to the probe changes as well. The changing particle flux leads to fluctuations in the probe's 
measured floating potential. By recording the floating potential fluctuations across two tips of the probe, the local electric field is determined and the wavelength of the travelling electrostatic wave can be determined. The local electric field calculation comes from $E=\Delta \varphi / d$, where $\Delta \varphi=\varphi_{1}\left(x_{1}\right)-\varphi_{2}\left(x_{2}\right)$ is the difference in floating potential at the location of the two probe tips and $d=x_{1}-x_{2}$ is the spatial separation of the two probe tips. For a measured phase difference of $\Delta \theta$ and the probe tip separation $d$ between the two probe tips, the wave number of a travelling wave is given by $k=\Delta \theta / d$. A power spectrum calculated from the fluctuating floating potential time series data provides a measure of the strength of the fluctuations at the frequencies of interest. By knowing the frequencies and the wavelengths of the wave, a dispersion relation ( $\omega$ versus $k$ ) for the wave is determined.

\subsubsection{Electrostatic Probe Design}

The electrostatic probe used to collect data in HELIX consists of four tungsten $0.55 \mathrm{~mm}$ diameter tips. Fig. 3.5 shows the orientation of the tips in a 4-bore alumina shaft. Using the numbering system in the figure, each tip has the following exposed tip lengths: 1.) $3.04 \mathrm{~mm}, 2$. $3.04 \mathrm{~mm}$, 3.) $2.67 \mathrm{~mm}$, and 4.) $3.07 \mathrm{~mm}$. The distances between the labeled tip numbers are given in Table 3.1. The tungsten tips protrude from an alumina shaft that is $2.38 \mathrm{~mm}$ in diameter and extends $69.69 \mathrm{~mm}$ from the $\mathrm{BN}$ cap. The probe was located $50 \mathrm{~cm}$ downstream the antenna at location C shown in Fig. 2.2. 


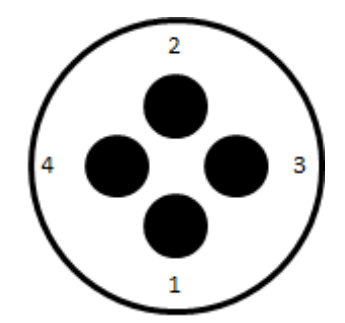

Figure 3.5. End view of the electrostatic double probe. The dark portions represent the locations of the tungsten tips in the 4-bore alumina.

\begin{tabular}{|c|c|}
\hline Tip Numbers & Distance (mm) \\
\hline 3 and 1 & 1.92 \\
\hline 3 and 2 & 1.92 \\
\hline 3 and 4 & 2.65 \\
\hline 4 and 1 & 2.15 \\
\hline 4 and 2 & 2.18 \\
\hline 2 and 1 & 2.79 \\
\hline
\end{tabular}

Table 3.1. Distances between the tips of the electrostatic multi-tip probe used in HELIX.

A second electrostatic probe was used to collect data downstream in LEIA and it consisted of only two tungsten $0.55 \mathrm{~mm}$ diameter tips in two 1-bore alumina shafts. One tip has an exposed tip length of $1.94 \mathrm{~mm}$ and an alumina shaft length of $66.72 \mathrm{~mm}$ from the exposed tungsten to the BN cap. The other has a $1.98 \mathrm{~mm}$ tip length and an alumina shaft length of 66.14 $\mathrm{mm}$ from the exposed tungsten to the BN cap. The two tungsten tips are separated by $3.9 \mathrm{~mm}$. This probe was located $80 \mathrm{~cm}$ from the base of the HELIX-LEIA junction at location G shown in Fig. 2.2. 
Inside the base of the $\mathrm{BN}$ cap of both electrostatic probes, the tungsten wires are connected with beryllium copper inline barrel connectors to the wires that bring the signals out to the vacuum BNC feedthrough. Thermoflex is used to cover the signal wires and keep them from touching inside the probe shaft. The signals from the selected tips are sent to a digitizer or an oscilloscope and stored on a computer for further processing. For these experiments, the probes were operated in either a one-tip or two-tip configuration. In the two-tip configuration, the tips would either be oriented parallel or perpendicular to the background magnetic field as shown in Fig 3.6.

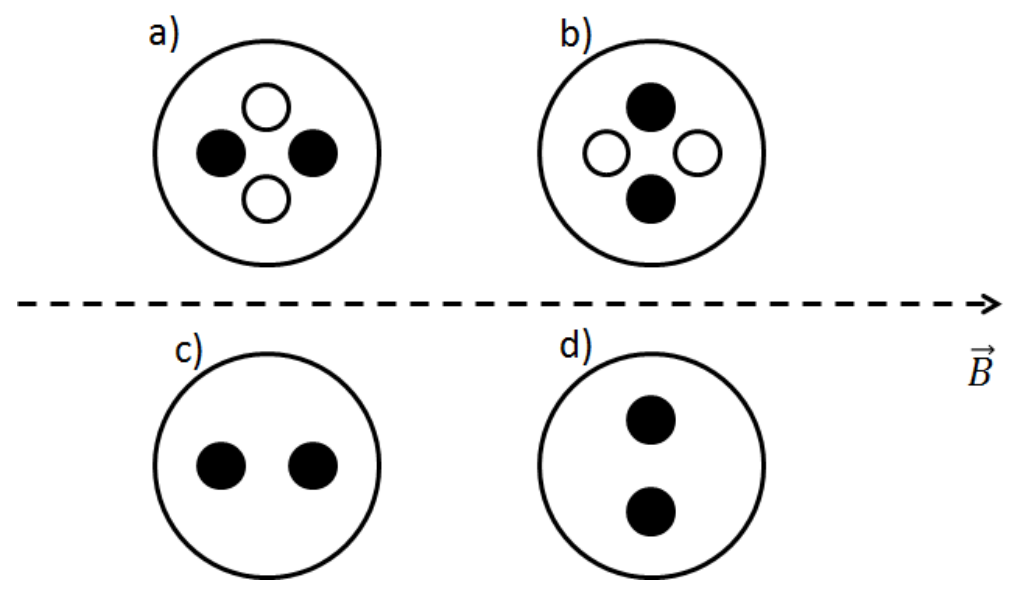

Figure 3.6. Possible electrostatic probe orientations with respect to the direction of the magnetic field: for HELIX multi-tip probe (a) parallel, (b) perpendicular and for LEIA double probe (c) parallel, (d) perpendicular.

\subsubsection{Electrostatic Probe Analysis}

Identification of waves in a plasma is typically accomplished through measurements of the wave frequency, propagation direction, and wavelength. The wavelength of a wave in a plasma is determined from probe measurements by measuring the phase difference between 
measurements performed at different spatial locations. Real signals contain noise and/or multiple wave harmonics. The cross power spectrum of the two measured time series is used to determine the phase difference for specific wave frequencies. The cross power spectrum of two time series is the product of the Fourier transform (FT) of one time series and the complex conjugate of the FT of the other time series. Let the two time series be represented by $f_{1}\left(x_{1}, t\right)$ and $f_{2}\left(x_{2}, t\right)$. The FT of each series is defined as

$$
\Phi_{1}\left(x_{1}, \omega\right)=\int_{-\infty}^{\infty} f_{1}\left(x_{1}, t\right) \cos (\omega t) d t-i \int_{-\infty}^{\infty} f_{1}\left(x_{1}, t\right) \sin (\omega t) d t
$$

and

$$
\Phi_{2}\left(x_{2}, \omega\right)=\int_{-\infty}^{\infty} f_{2}\left(x_{2}, t\right) \cos (\omega t) d t-i \int_{-\infty}^{\infty} f_{2}\left(x_{2}, t\right) \sin (\omega t) d t
$$

where $x_{1}$ and $x_{2}$ are the respective probe tip locations. The cross power spectrum, $P_{12}$, of the two signals is the product of Eq. 3.10 and the complex conjugate of Eq. 3.11;

$$
P_{12}(d, \omega)=\Phi_{1}\left(x_{1}, \omega\right) \Phi_{2}^{*}\left(x_{2}, \omega\right)
$$

where $d=x_{1}-x_{2}$ is the spatial separation of the probe tips. Expanding Eq. 3.12 and collecting the real and imaginary terms,

$$
\begin{aligned}
P_{12}(d, \omega)=( & \left.\operatorname{Re}\left\{\Phi_{1}\right\} \operatorname{Re}\left\{\Phi_{2}\right\}+\operatorname{Im}\left\{\Phi_{1}\right\} \operatorname{Im}\left\{\Phi_{2}\right\}\right) \\
& +i\left(\operatorname{Im}\left\{\Phi_{1}\right\} \operatorname{Re}\left\{\Phi_{2}\right\}-\operatorname{Re}\left\{\Phi_{1}\right\} \operatorname{Im}\left\{\Phi_{2}\right\}\right)
\end{aligned}
$$


In the complex plane, the angle between the real and imaginary vectors of the cross power spectrum is the phase difference of the two time series:

$$
\Theta(\omega)=\tan ^{-1}\left(\frac{\left(\operatorname{Im}\left\{\Phi_{1}\right\} \operatorname{Re}\left\{\Phi_{2}\right\}-\operatorname{Re}\left\{\Phi_{1}\right\} \operatorname{Im}\left\{\Phi_{2}\right\}\right)}{\left(\operatorname{Re}\left\{\Phi_{1}\right\} \operatorname{Re}\left\{\Phi_{2}\right\}+\operatorname{Im}\left\{\Phi_{1}\right\} \operatorname{Im}\left\{\Phi_{2}\right\}\right)}\right)
$$

These calculations implicitly assume a large signal to noise ratio. If the signal to noise ratio is low, ensemble averages of cross power spectra can significantly improve the precision of the phase measurements. The random error decreases as $1 / \sqrt{M}$ where $M$ is the number of data samples obtained. ${ }^{16}$

Although maximizing $M$ is desirable, the need for larger data records presents a challenge. To address this issue, a Lecroy WaveRunner ${ }^{\mathrm{TM}} 604 \mathrm{Zi}$ oscilloscope with the ability to perform onboard averages of fast Fourier transforms (FFTs) was used. Briefly, Fourier analysis of discrete signals is accomplished through the discrete Fourier transform (DFT) and the FFT is a recursive algorithm that implements DFTs more efficiently. A more detailed description of DFTs and FFTs can be found in Section 4.2.1. The oscilloscope was set to record the two time series and then store averages of the real and imaginary parts of the cross power spectra. After collecting 500 averages of both quantities, the average cross power spectrum was constructed,

$$
\begin{aligned}
\overline{P_{12}}(\Delta x, \omega)= & \left(\overline{\operatorname{Re}\left\{\Phi_{1}\right\} \operatorname{Re}\left\{\Phi_{2}\right\}+\operatorname{Im}\left\{\Phi_{1}\right\} \operatorname{Im}\left\{\Phi_{2}\right\}}\right) \\
& +i\left(\overline{\operatorname{Im}\left\{\Phi_{1}\right\} \operatorname{Re}\left\{\Phi_{2}\right\}-\operatorname{Re}\left\{\Phi_{1}\right\} \operatorname{Im}\left\{\Phi_{2}\right\}}\right)
\end{aligned}
$$

without the need to store all of the time series measurements.

Even when using the cross power spectrum technique, aliasing in frequency and space remains a concern. In power spectrum measurements at a single spatial location, aliasing occurs 
when a periodic signal's frequency is larger than the Nyquist frequency $\left(v_{\text {Nyquist }}\right.$ is half the sampling frequency) of the data acquisition system. In an FFT of an aliased time series measurement, the periodic signal spectral power will appear downshifted in frequency. Additional spectral peaks at harmonics of the artificially downshifted frequency are also likely to appear in the FFT. For the sampling rate of $100 \mathrm{MHz}, v_{N y q u i s t}$ is $50 \mathrm{MHz}$. The fast digitization rate of $100 \mathrm{MHz}$ was chosen so that the large periodic signal at the rf antenna frequency of 9 $\mathrm{MHz}$ and its first harmonic are well-resolved. Above $25 \mathrm{MHz}$, the amplitude of the fluctuation spectrum is small and there was no need for active filtering of signals above the Nyquist frequency.

Spatial aliasing occurs when the wavelength of the wave is smaller than the separation distance between the two tips. Fig 3.7 depicts a situation where the measured phase differences of two waves are the same even though their wavelengths are not. ${ }^{17}$ The minimum resolvable wavelength is determined by the probe tip separation distance: $\lambda_{\min }=2 d$ where $d$ is the probe separation distance. For a maximum phase difference of $\pi$ and a tip separation of $0.279 \mathrm{~cm}$ (largest gap reported in Table 3.1), the maximum measureable wave number is $\pm 5.63 \mathrm{rad} / \mathrm{cm}$.
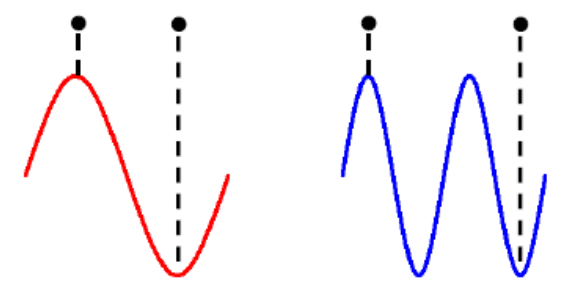

Figure 3.7. An illustration of spatial aliasing for a pair of fixed probes. The black dots and dashed lines represent the spatial location of the probe tips while the color sinusoids represent the wave amplitudes at an instant in time. Figure obtained from Ref. [17]. 
Some electrostatic probe studies were able to take advantage of newly constructed highspeed, differential signal amplifiers (shown in Fig. 3.8). With a design courtesy of the UWMadison MST group, WVU constructed these filtered amplifiers to study lower frequency signals ( tens of $\mathrm{kHz}$ ) while high frequency signals were present. The amplifiers have three parallel outputs with input filter corner frequencies of $215 \mathrm{kHz}, 615 \mathrm{kHz}$, and $1.9 \mathrm{MHz}$, respectively. For the measurements presented here, the $215 \mathrm{kHz}$ channel was used.

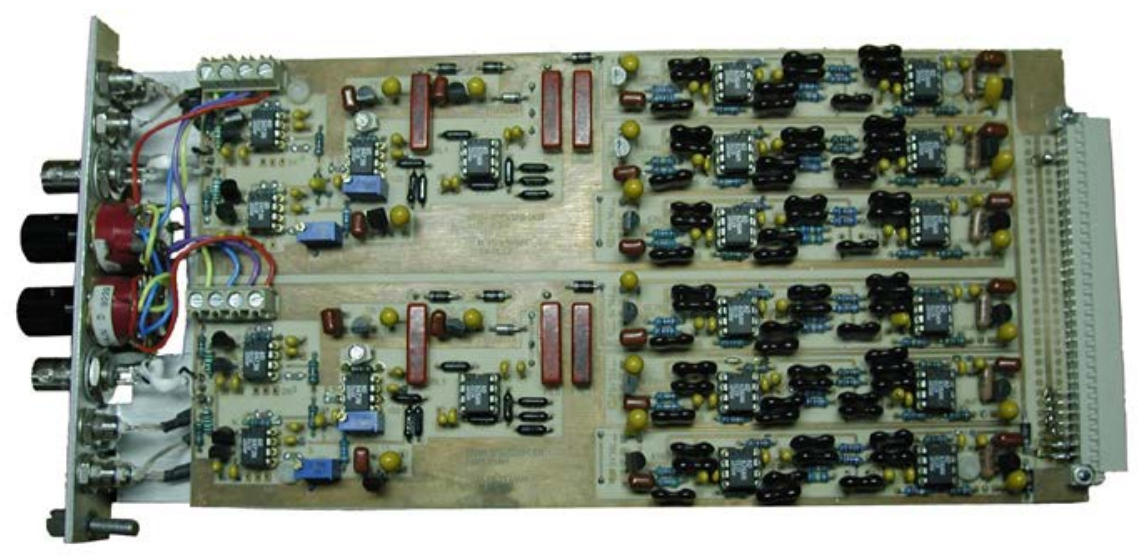

Figure 3.8. High speed differential amplifier. Design courtesy of the UW-Madison MST group.

\subsection{Retarding Field Energy Analyzer (RFEA)}

Retarding field energy analyzers (RFEAs) have been used over the last several decades to measure ion energy distributions (IEDs) in a variety of plasma reactor configurations. Chakraborty Thakur in Ref. [18] details the previous RFEA work of the WVU HELIX group while including a review of the history and theory of this diagnostic. A brief overview of the RFEA diagnostic is presented here, with the focus on a new probe design and data analysis 
techniques that resulted from a recent collaboration with the Space Physics Group at the University of Tromsø (UiT).

All RFEAs share the basic principle of placing a grid in front of a beam of ions and increasing the potential of that grid until the ion beam flux stops. The integral of the IED is obtained by measuring the current of the collected ions as a function of the grid potential. The IED is then obtained by detailed analysis of the current-potential characteristic. The IED, the density of ions in energy space as a function of energy for a given point in space and a moment in time, contains nearly the same information as the ion velocity distribution function (IVDF), the density of ions in phase space as a function of velocity for a given point in space and a moment in time.

\subsubsection{RFEA Theory}

The schematic in Fig. 3.9 shows the working principles of a RFEA. This particular schematic is based on the RFEA used by WVU in previous experiments; its construction was completed by WVU researchers and based on design schematics provided by the Australia

National University (ANU) group. ${ }^{18,19,20}$ Particles follow the magnetic field lines towards the entrance slit in the probe that admits plasma. The parallel component of the ion flux is measured by making the plane of the aperture normal to the local magnetic field. A grid just behind the entrance slit (or in some designs, the entrance slit itself) is biased to a sufficiently large and constant negative potential $\left(\Phi_{e}\right)$ to repel most of the electrons entering from the plasma. The next grid the ions face is biased with an applied discriminator potential $\left(\Phi_{D}\right)$ that ranges from zero to a large positive value to repel ions. Ions must have a kinetic energy larger than $e \Phi_{D}$ in order to cross this retarding grid. The third grid after the entrance slit is biased to a constant 
negative potential $\left(\Phi_{S}\right)$ to suppress any secondary electrons produced by the ion bombardment on the collector and to repel any secondary electrons created by ion impact on the second grid. The collector current decreases as the potential on the discriminator increases and more ions are repelled.

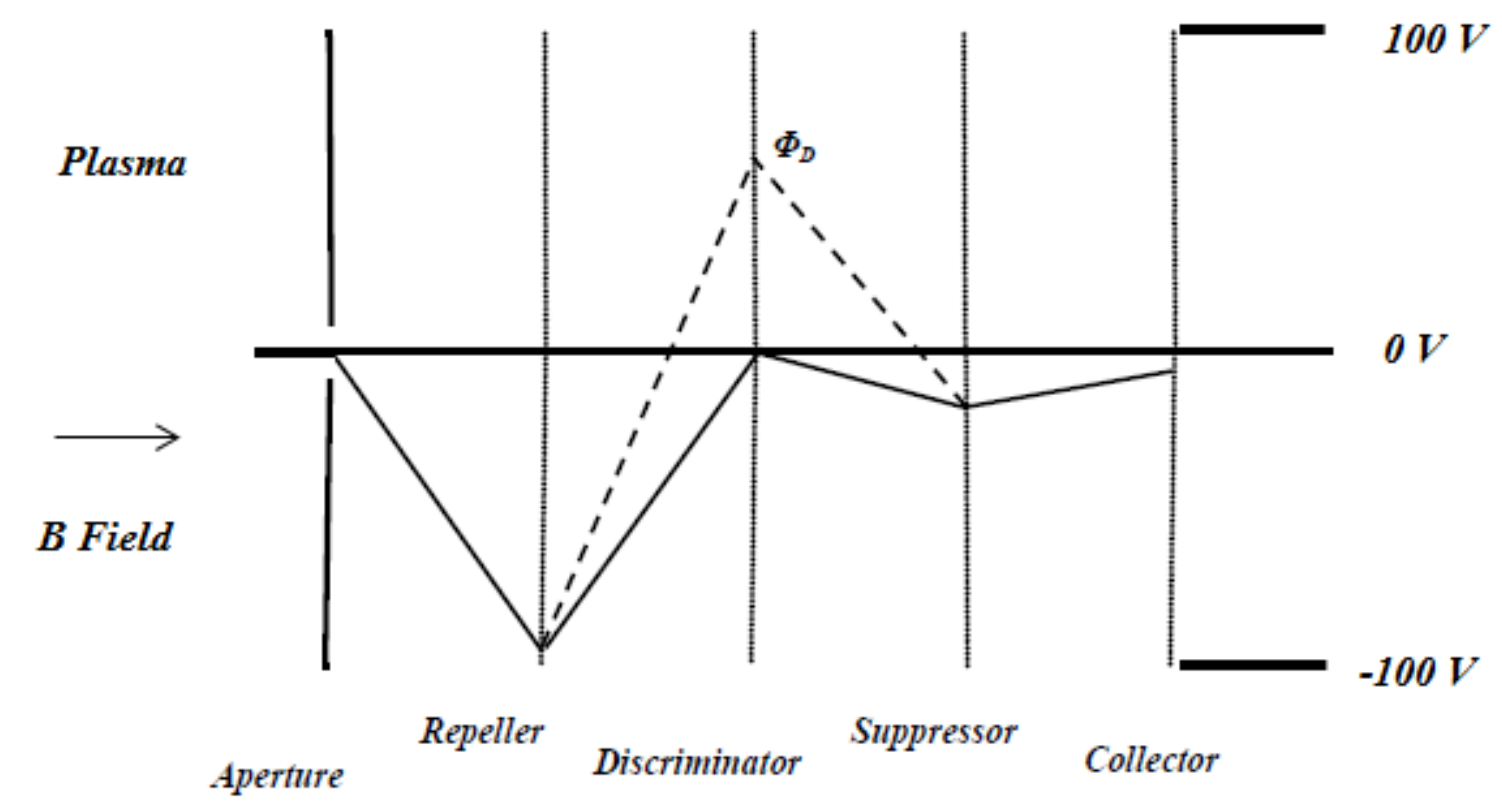

Figure 3.9. The WVU RFEA schematic. The aperture is on the left and the vertical dotted lines represent the four grids. The dark line shows typical voltages applied to each grid. The dashed line shows the typical range of voltages applied to the discriminator grid. This figure is adapted from Ref. [18].

There are several theories that describe how the collector current measured as a function of the discriminator potential depends on the energy of the ions and relates to the ion velocity distribution function. ${ }^{18,21,22,23,24,25}$ The typical, and incorrect, approach is to assume that first derivative of the collector current $\left(I_{C}\right)$ with respect to the varying potential $\left(\Phi_{D}\right)$ applied to the retarding grid gives the ion velocity distribution $(f(v))$. To illustrate this relationship, consider a 
one dimensional IVDF while dropping the directional subscript. For a given point in space and moment in time, the total ion density is computed with either the IED $(g(E))$ or the IVDF:

$$
\int_{-\infty}^{\infty} g(E) d E=\int_{0}^{\infty} d n=\int_{-\infty}^{\infty} f(v) d v
$$

Only the kinetic energies of ions with mass $m$ are considered. Consequently, the variables of integration are related through

$$
d E=d\left(\frac{1}{2} m v^{2}\right)=m v d v
$$

and the IVDFs and IEDs of the ions entering the RFEA are related through a constant of integration,

$$
f(v)=(m v) g(E)
$$

The total ion current density in the entrance plane of the analyzer $\left(I_{\text {ion }}\right)$ in this one dimensional case is given by

$$
I_{\text {ion }}=e \int v f(v) d v=\frac{e}{m} \int f(v) d E
$$

With RFEAs, ions with energies larger than the potential applied to the discriminator grid pass through. Letting $E \rightarrow \varepsilon$ to allow for a smooth transition to the commonly used notation and redefining “ $E$ ” to be the minimum energy for ions entering the RFEA, Eq. 3.19 becomes 


$$
I_{\text {ion }}=\frac{e}{m} \int f\left[\left(\frac{2 \varepsilon}{m}\right)^{1 / 2}\right] d \varepsilon
$$

All the ions reaching the entrance slit pass unhindered through the grids when the discriminator potential $\left(\Phi_{D}\right)$ is zero i.e. it is grounded and contribute to the current collected by the collector, $I_{C} . I_{C}$ decreases as $\Phi_{D}$ is swept from zero to more positive values. At the cutoff energy, the minimum energy needed to overcome the discriminator bias, the current collected by the collector is

$$
I_{C}(E)=\frac{e}{m} \int_{E}^{\infty} f\left[\left(\frac{2 \varepsilon}{m}\right)^{1 / 2}\right] d \varepsilon
$$

\subsubsection{RFEA Design and Schematics}

Fig. 3.10 shows the schematics of the RFEA probe for the experiments reported here. The probe was designed and constructed by researchers from the UiT and placed on the WVU HELIX-LEIA plasma experiment at location G in Fig 2.2. The experimental goal was to make a comparison of two plasma diagnostics, the RFEA and laser induced fluorescence on identical plasma parameters that result in a current free double layer. The UiT RFEA schematic is shown in Fig. 3.10 along with the two configurations used. The probe uses three grids and a collector. The front grid is either floating or grounded. The repeller grid (R) is biased to $-100 \mathrm{~V}$ to repel electrons. The discriminator (D) discriminates ions based on velocity and a collector (C) is biased to $-9 \mathrm{~V}$ to collect the ion current. The suppressor grid was eliminated from these configurations because there was not a clear difference between measurements that did or did not 
employ a suppressor grid. For some measurements, the positions of the repeller and the discriminator were swapped to investigate any effects of ion focusing into the collector.

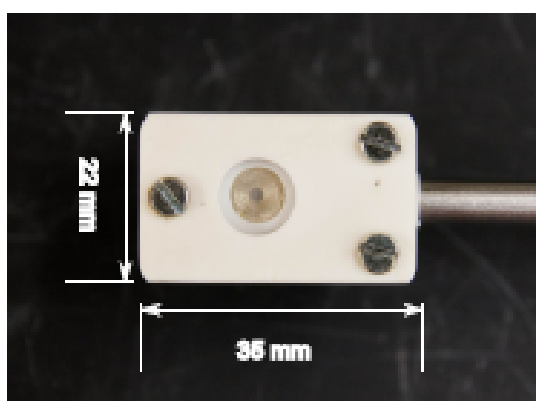

(a)

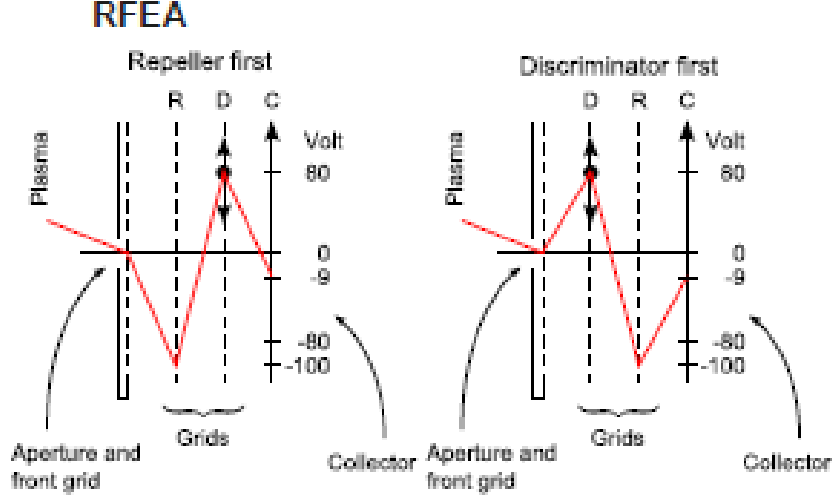

(b)

(c)

Figure 3.10.: a) The UiT RFEA. b). Schematics of the grids in the RFEA for the repeller in front of the discriminator. c). Schematics of the grids in the RFEA for the discriminator in front of the repeller. Both configurations have the front grid either floating or grounded.

\subsubsection{RFEA Data Analysis}

To extract the IVDF from the measured current versus discriminator curve, the measurements are fitted to an analytic function that describes the expected current for a given discriminator voltage based on some assumptions about the IVDF, e.g., one or two populations, flow or no flow, etc.. For a velocity distribution consisting of two ion populations, one a background, stationary population accelerated into the RFEA by the difference between the 
grounded front grid of the RFEA and the local plasma potential and the other an ion beam population, the collected current is described by

$$
\begin{aligned}
I_{c}\left(\Phi_{D}\right)=\sqrt{\frac{e^{2}}{2 m \pi}}\left[( \frac { n _ { p } } { 2 } ) \left\{\sqrt{T_{p}} e^{-\left(\frac{\left(\sqrt{e \Phi_{D}-e \Phi_{p}}-\sqrt{E_{p}}\right)^{2}}{T_{p}}\right)}\right.\right. \\
\left.+\sqrt{E_{p} \pi} \operatorname{erfc}\left[\frac{\left(\sqrt{e \Phi_{D}-e \Phi_{p}}-\sqrt{E_{p}}\right)^{2}}{T_{p}}\right]\right\} \\
+\left(n_{b}\right)\left\{\sqrt{T_{b}} e^{-\left(\frac{\left(\sqrt{e \Phi_{D}-e \Phi_{p}}-\sqrt{E_{b}}\right)^{2}}{T_{b}}\right)}\right. \\
\left.+\sqrt{E_{b} \pi} \operatorname{erfc}\left[\frac{\left(\sqrt{e \Phi_{D}-e \Phi_{p}}-\sqrt{E_{b}}\right)^{2}}{T_{b}}\right]\right\} .
\end{aligned}
$$

where the $p$ subscript denotes a background population, the $b$ subscript denotes a beam population, $n$ is the density, $T$ is the ion temperature, $m$ is the ion mass, $E_{p}$ is drift energy acquired by the bulk ion population as it is accelerated to the RFEA through the sheath in front of the grounded front grid, and $E_{b}$ is energy of the ion beam population. Fig 3.11 shows a comparison between the results of a fit of Eq. 3.24 to a RFEA measurement and a LIF measurement of the IVDF at the same location and for the same plasma parameters. The red curve is the IVDF obtained from the fit and shown in units of laser frequency for comparison with the LIF measurement. The green line is the LIF measurement and clearly shows the presence of an ion beam population in addition to a background ion population. The two measurement techniques are in good agreement. 


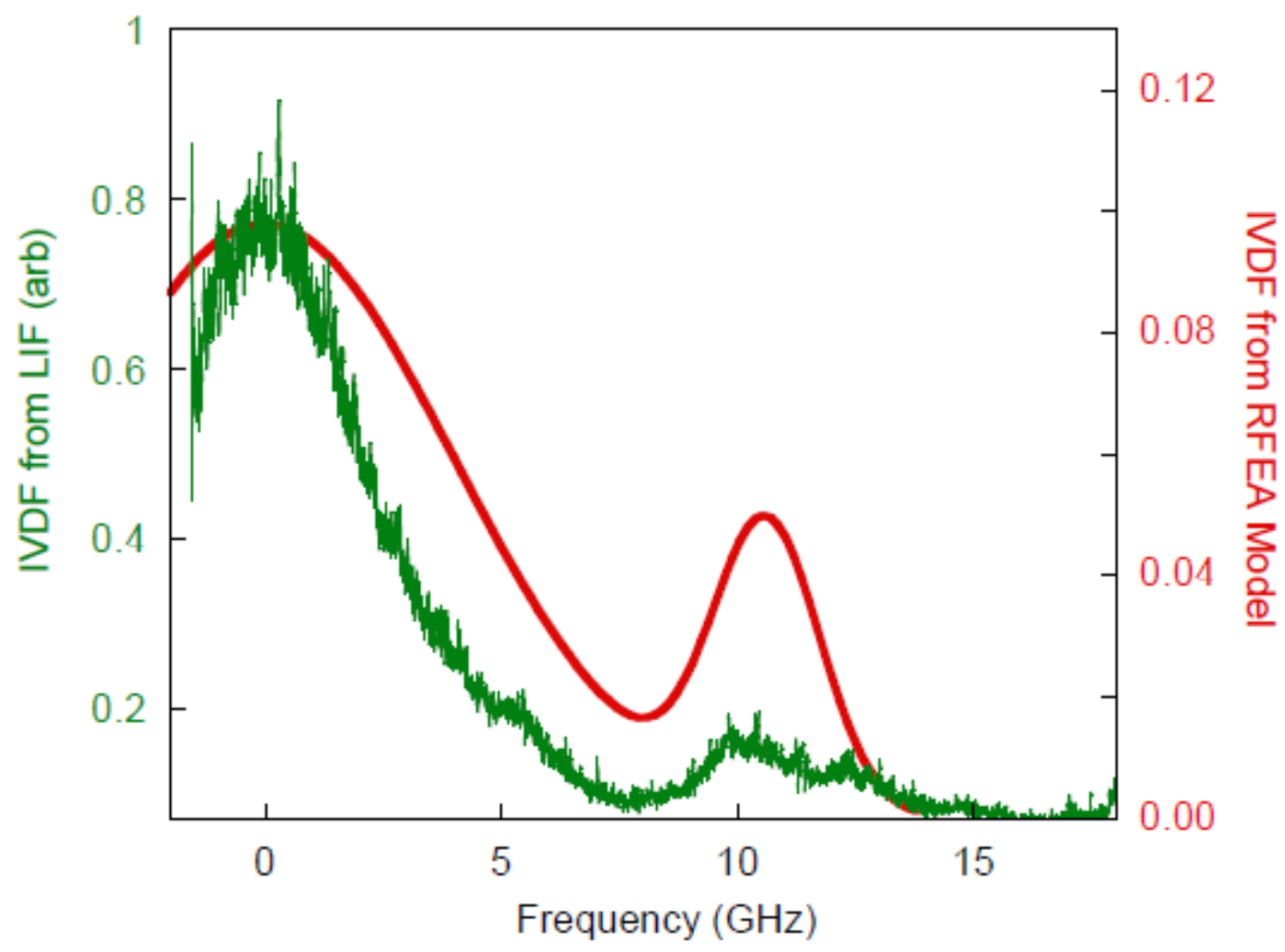

Figure 3.11. The two ion velocity distributions obtained from fitting Eq. 3.24 to the RFEA data (red) compared to LIF measurements (green) of the IVDF at the same axial location (position F in Fig. 2.2). The Figure is obtained from Ref. [18].

An alternate approach for analysis of the RFEA data is to fit the I-V trace with a Savitzky-Golay-filter. A Savitzky-Golay-filter is a windowing filter that fits a polynomial centered on every data point. Then the derivative of the fitted function is plotted against the discriminator voltages to approximate the IED. Comparisons of LIF measurements of beam energies to RFEA measurements of the IED based on the Savitzky-Golay-filter are presented in Section 5.4. 


\subsection{Continuous Wave Laser Induced Fluorescence}

A thorough review of the history of laser induced fluorescence (LIF) for dye laser systems can be found in Ref. [26]. Only a brief overview will be given here. In LIF, a laser is tuned to a natural absorption line of an atom or ion to induce emission from the upper pumped state to either the same initial state (resonant LIF) or a different third state (non-resonant LIF). In 1966, Yardley and Moore were the first to observe LIF from molecules other than the lasing medium of a laser. ${ }^{27}$ Almost ten years later, Stern and Johnson provided the first experimental evidence of LIF in a plasma using a single frequency argon laser and creative positioning of the laser relative to the target plasma. ${ }^{28}$ Hill et al. ${ }^{29}$ made use of a non-resonant transition and were the first to use the capability to tune the laser wavelength to obtain velocity selective LIF measurements in 1983. Spectroscopic techniques that rely on measurements of the emission line shape suffer from being limited by the resolution of the resolving instrument, typically a spectrometer. Hill et al. instead measured the absorption line shape with a velocity resolution determined by the natural line width of the laser and the ion transition. The LIF system for HELIX-LEIA employs the techniques developed by Hill et al. along with a non-resonant scheme so as to easily differentiate between the induced emission and laser light.

The width of an absorption line depends on a variety of possible broadening mechanisms. Typical broadening mechanisms include the natural line width, Stark broadening, power broadening, Doppler broadening and Zeeman broadening. Doppler broadening and Zeeman broadening are the two most significant broadening mechanisms in the HELIX-LEIA experiment. All other possible broadening mechanisms are negligible for the parameters of these 
experiments. ${ }^{30}$ It is through the Doppler broadening of the absorption line that the velocity distribution function (VDF) is determined.

A LIF measurement of a VDF in a plasma is obtained by scanning the frequency of a narrow line width, tunable laser across an absorption line of an ion or neutral species while the intensity of the fluorescent emission from the excited state is measured as a function of the laser frequency. Typically, only metastable excited states or ground states are populated enough to be used for the initial state. Moving ions or neutrals absorb the Doppler shifted laser light in their rest frame, pumping an electron to a higher energy, typically short-lived, excited state. Later, the electron spontaneously decays into a lower energy level. By using injection and collection optical paths that cross in only one spot, LIF measurements are localized to a specific location in the plasma. LIF measurement spatial resolutions of $\sim 1 \mathrm{~mm}^{-3}$ are easily accomplished. Analysis of the VDF measurement yields the ion or neutral temperature, the density of the absorbing state (if the LIF system is fully calibrated), and the net drift velocity. The Doppler broadening of an ion absorption line for a Maxwellian distribution is given by

$$
I(v)=I_{o} \exp \left(\frac{-m\left(v-v_{0}^{*}\right)^{2} c^{2}}{2 k_{B} T v_{o}^{2}}\right),
$$

where $I(v)$ is the absorbed photon flux as a function of frequency $v, I_{o}$ is the maximum photon flux absorption and $v_{o}^{*}=v_{o}+v_{o} v / c$ is the proper frequency of the transition when viewed from the laboratory frame.

Zeeman splitting yields linearly polarized $\pi$ lines $(\Delta m=0)$ and circularly polarized $\sigma$ lines $(\Delta m= \pm 1)$ for absorption between the initial and the upper states in the presence of strong magnetic fields. Fig. 3.12 shows the details of typical Zeeman splitting for the primary 
$611.6616 \mathrm{~nm}$ absorption line of argon. The $\pi$ lines are symmetrically distributed around the zero magnetic field transition while the $\sigma$ lines include two clusters of lines, $\sigma+$ and $\sigma-$. The amplitude envelope of each of $\sigma+$ or $\sigma$ - clusters is asymmetric. However, each cluster is distributed symmetrically around the central line which denotes the zero magnetic field transition. The frequency shift of each cluster from the central line depends linearly on the magnetic field strength. The strength of the magnetic field at the measurement location can be determined from the measured shift of the $\sigma$ clusters.

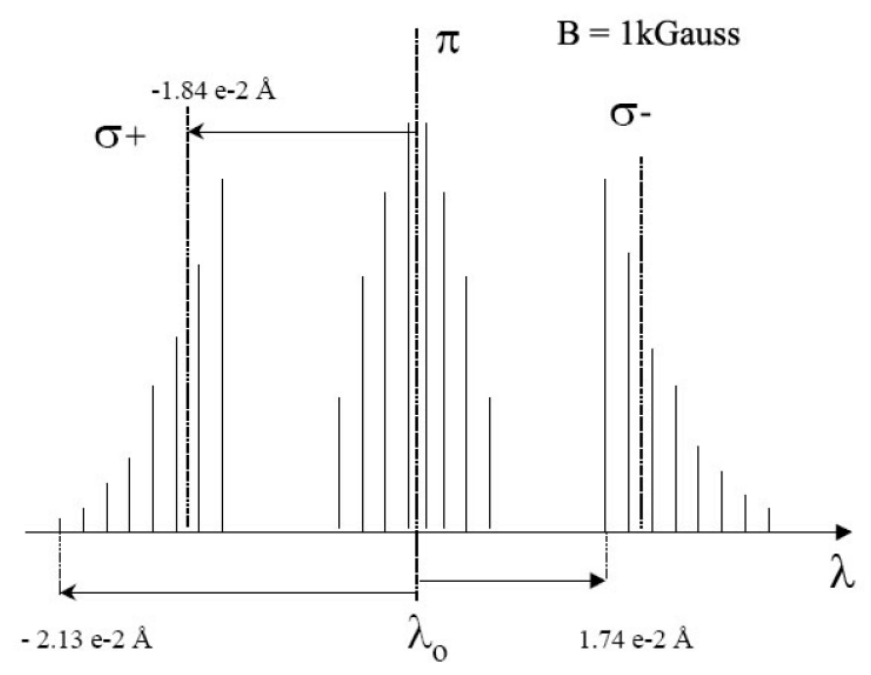

Figure 3.12. Schematic of the $\sigma$ and $\pi$ transitions for the $611.6616 \mathrm{~nm}$ argon ion absorption line. The height of each line corresponds to the statistical weighting of each transition as a function of wavelength. Figure obtained from Ref. [32].

Each Zeeman component is also Doppler broadened in a thermal distribution of particles. For large magnetic fields, accurate temperature measurements are only possible if the LIF signal is de-convolved into each of its individual Zeeman components. ${ }^{31}$ The Zeeman splitting of $\pi$ 
lines and the two sets of $\sigma$ lines in HELIX-LEIA is much less than Doppler broadening of these same lines. ${ }^{32}$ This allows each cluster of $\sigma$ lines and the cluster of $\pi$ lines to be treated as a single Doppler broadened line shifted from the proper frame wavelength by the statistically weighted average Zeeman shift of the individual lines in the cluster (zero shift in the case of the cluster of $\pi$ lines). VDFs parallel and perpendicular to the background magnetic field lines are selected by the choice of the laser injection relative to the magnetic field. For perpendicular injection, the laser is polarized parallel to the magnetic field, allowing only the $\pi$ lines to be excited. For parallel injection, the laser is circularly polarized so that only one cluster of $\sigma$ lines is excited.

\subsubsection{LIF of Argon II with a Dye Laser}

Two continuous wave ring dye laser systems were used to obtain the LIF data presented in this work: a Coherent 899 ring dye laser and a Matisse-DR ring dye laser (with which the vast majority of these data were collected). A complete description of the Coherent 899 ring dye laser can be found in Ref. [26] while the Matisse-DR system is described here. The Matisse-DR ring dye laser was introduced as an upgrade to the Coherent 899 laser with increases in power, scanning range and laser control. The Matisse-DR dye laser has a line width of less than $20 \mathrm{MHz}$ RMS (root mean square) and it is pumped by a Millennia Pro 10s diode laser. The Millennia Pro 10s pumps the Matisse-DR ring dye laser using Rhodamine 6G dye that fluoresces from $550 \mathrm{~nm}$ to $660 \mathrm{~nm}$.

For LIF measurements of the argon IVDF, the LIF laser system is tuned to $611.6616 \mathrm{~nm}$ (vacuum wavelength) to pump the Ar II $3 d^{2} G_{9 / 2}$ metastable state to the $4 p^{2} F_{7 / 2}$ state, which then decays to the $4 \mathrm{~s}^{2} \mathrm{D}_{5 / 2}$ state by emitting $460.96 \mathrm{~nm}$ photons. The LIF scheme is shown in Fig 3.13. 


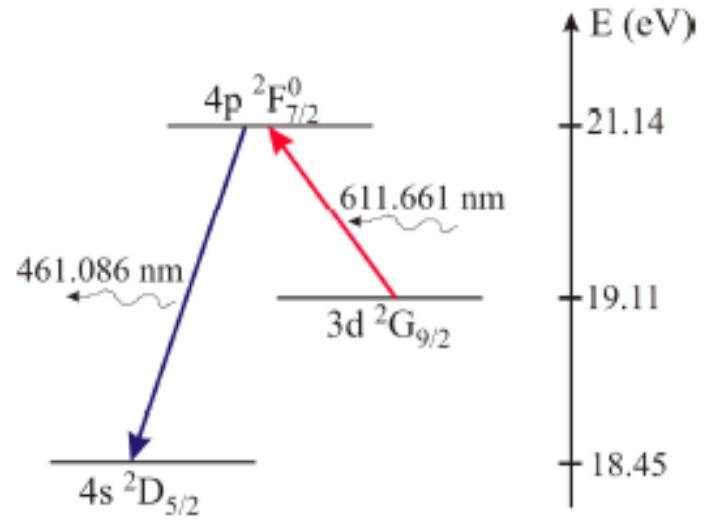

Figure 3.13. LIF scheme for Ar II using the ring dye laser.

The LIF system configuration used on WVU HELIX-LEIA is shown in Fig. 3.14. The Matisse-DR dye laser beam is split and 99\% is modulated with either an acousto-optic modulator $(\mathrm{AOM})$ at $40 \mathrm{kHz}$ or a mechanical chopper at $1 \mathrm{kHz}$. For good time resolution, time resolved experiments require more frequent modulated light pulses than the mechanical chopping can provide, while a mechanical chopper allows for more laser light to be injected into the plasma. Once modulated, the laser light is coupled into a multimode, non-polarization-preserving, optical fiber for transport from the laser laboratory to injection optics aligned along the magnetic axis of the helicon source-expansion chamber system. As the laser frequency is swept over as much as $60 \mathrm{GHz}$, the fluorescent emission from the pumped excited state is collected and transported by an optical fiber to a filtered (1 nm bandwidth around the fluorescence wavelength) narrowband, high-gain, Hamamatsu photomultiplier tube (PMT). Since the PMT signal is composed of background spectral radiation, electron-impact-induced fluorescence radiation, and electronic noise, a Stanford Research SR830 lock-in amplifier is used to eliminate signals uncorrelated with the laser modulation. Lock-in amplification is essential since electron-impact-induced emission 
is several orders of magnitude larger than the fluorescence signal. The remaining $1 \%$ of the dye laser beam is sent to another beam splitter for diagnostic purposes.

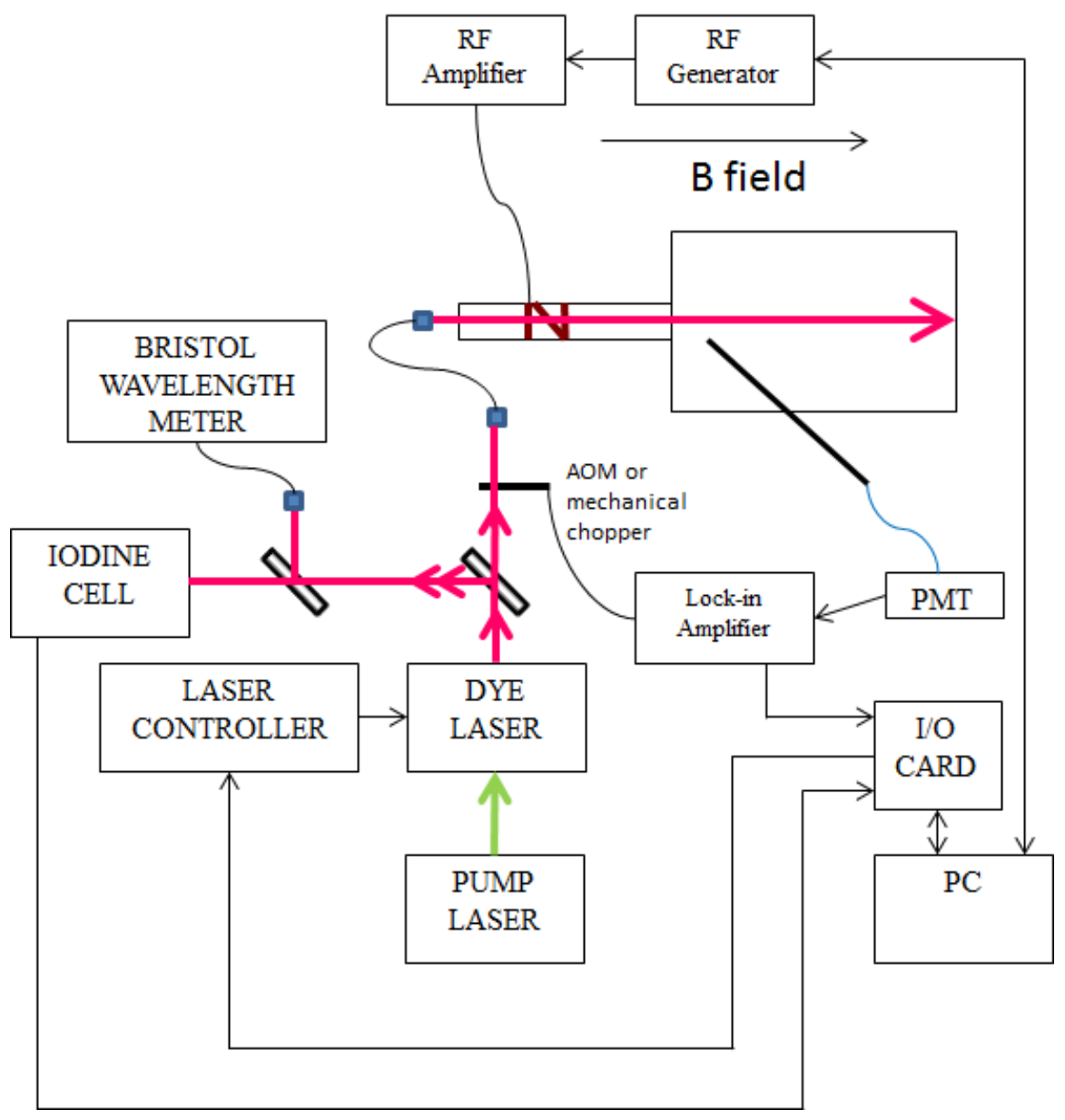

Figure 3.14. The continuous wave LIF diagnostic apparatus.

The diagnostic beam is split into two paths. One beam is coupled into a Bristol Instruments 621-VIS wavelength meter for laser wavelength measurements. The other beam passes through an iodine cell for a consistent zero-velocity reference measurement. Fluorescent emission from the iodine cell is detected with a photodiode for each frequency scan of the laser. The Salami reference iodine spectrum was compared to the experimentally obtained iodine spectra in the ranges of interest of each LIF scheme to identify the appropriate iodine lines to be 
used as a zero velocity reference for the LIF measurements. ${ }^{33}$ Fig. 3.15 shows that the iodine line closest to the zero velocity reference for the Ar II absorption line at $661.6616 \mathrm{~nm}$ (or 16348.91 $\mathrm{cm}^{-1}$ ) with a sufficiently strong intensity is the $16348.94 \mathrm{~cm}^{-1}$ line. The difference between the argon ion and the iodine line corresponds to a shift of $1.08 \mathrm{GHz}$.

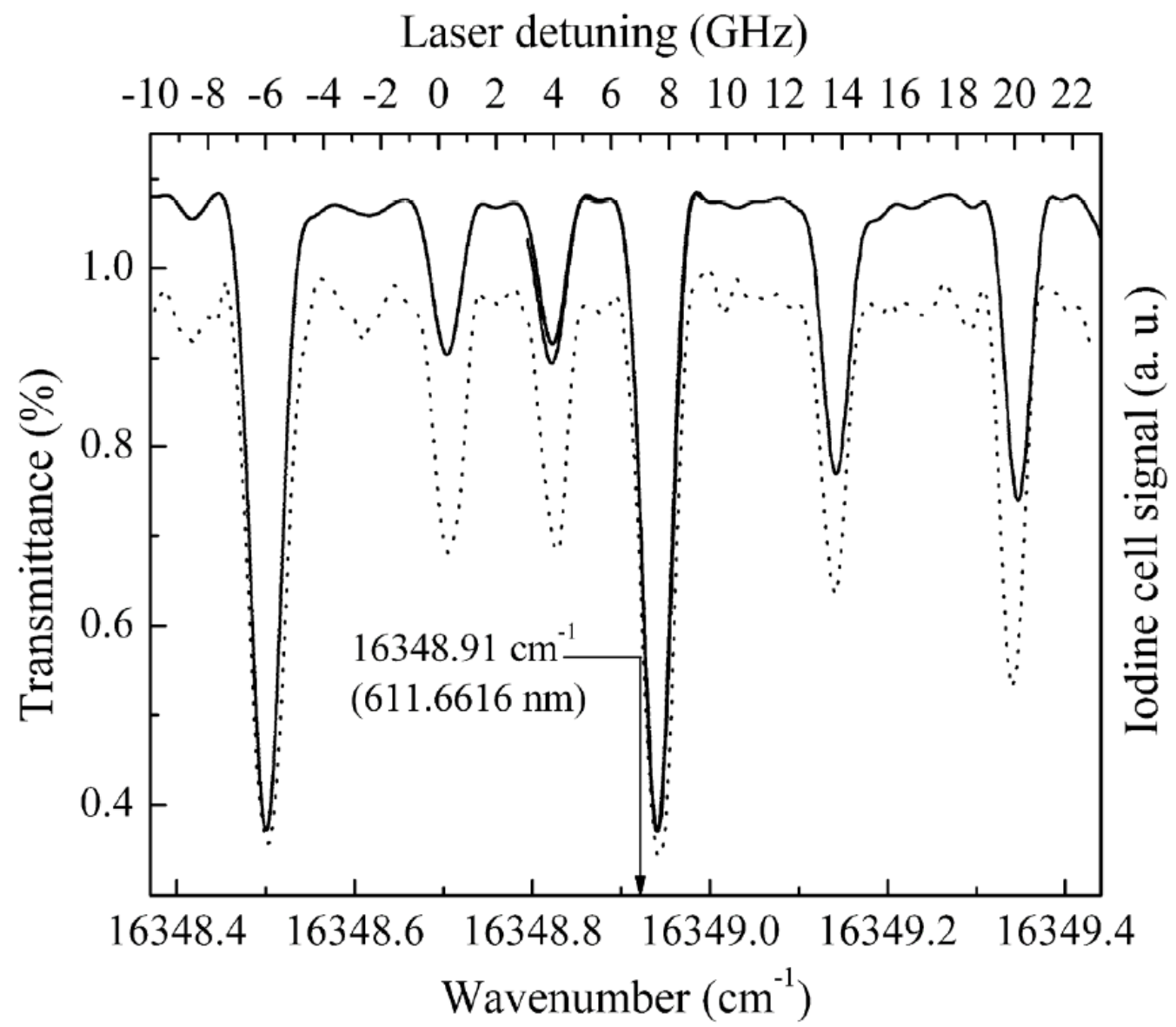

Figure 3.15. Iodine spectra for $\sim \pm 15 \mathrm{GHz}$ relative to $611.6616 \mathrm{~nm}$. This spectrum was obtained from two partially overlapping $20 \mathrm{GHz}$ wide laser scans represented by the solid line. As given in Ref. [33], the reference iodine spectrum in the spectral ranges of interest for Ar II is shown as the dotted line. Figure adapted from Ref. [11]. 
A typical LIF measurement of an Ar II IVDF in LEIA, $126 \mathrm{~cm}$ downstream of the antenna (position D in Fig. 2.2), is shown in Fig. 3.16. The bulk velocity for the Ar II IVDF is calculated from the absolute shift of the measured IVDF according to

$$
V=\lambda_{o} \Delta v_{\text {total }}
$$

where the velocity $V$ is in meters per second for the rest frame wavelength, $\lambda_{o}$, in nanometers and the total frequency shift, $\Delta v_{\text {total }}$, in gigahertz. The total frequency difference is equal to the difference between the LIF signal peak and the iodine reference line (1.46 GHz in this example) plus the $1.08 \mathrm{GHz}$ shift in the peak of iodine reference line from the non-shifted absorption line at $611.6616 \mathrm{~nm}$ while subtracting the Zeeman shift due to the magnetic field at the measurement location (for these experimental conditions, the $\sigma^{+}$Zeeman shift is $1.03 \mathrm{GHz}$ ). Thus, the total frequency shift is $1.51 \mathrm{GHz}$, corresponding to a bulk velocity of $\sim 925 \mathrm{~m} / \mathrm{s}$. The argon IVDF is well fit with a single Gaussian function, according to Eq. 3.25. The parallel ion temperature is determined to be $0.16 \mathrm{eV}$ from the full width at half maximum (FWHM). 


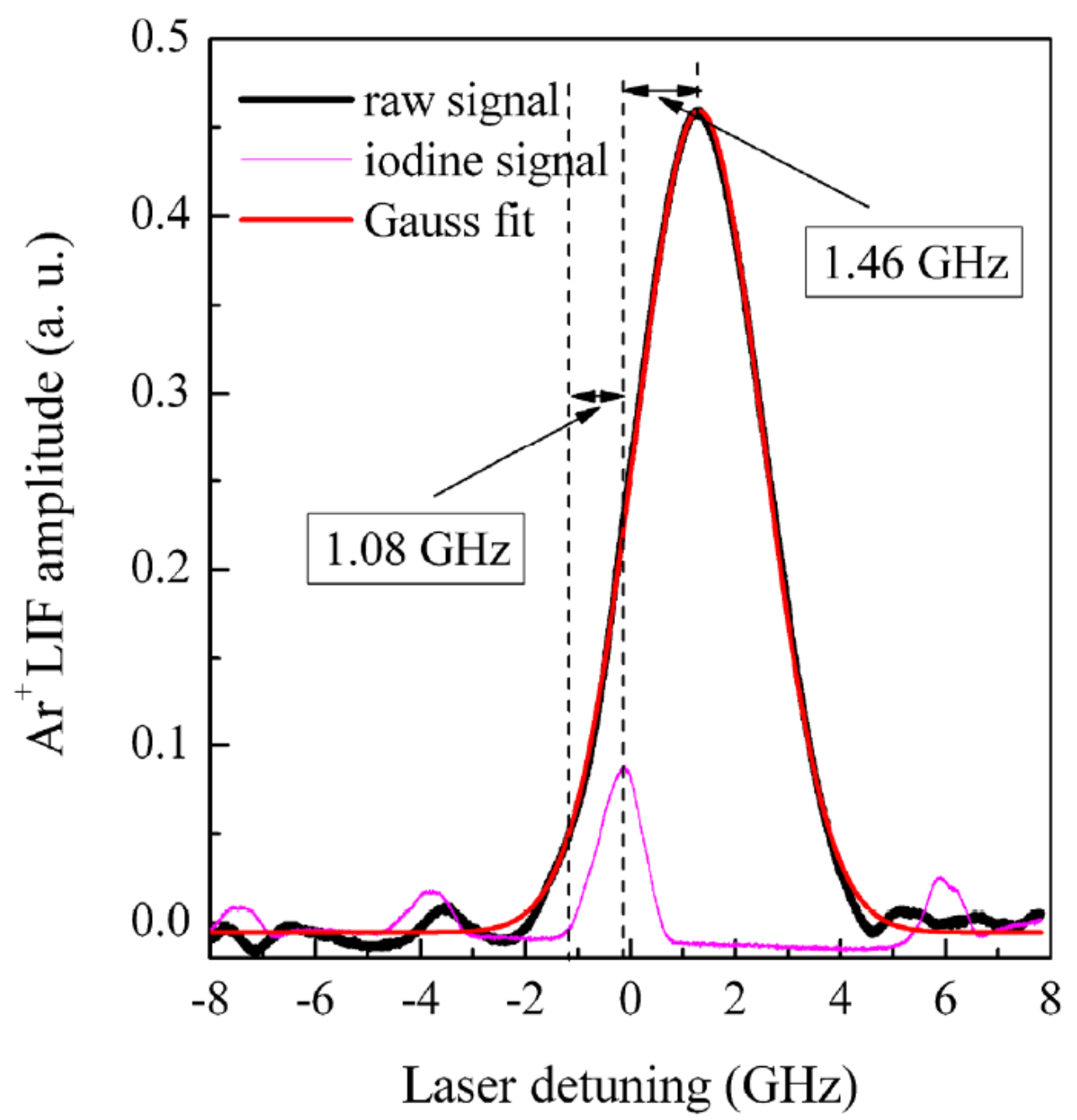

Figure 3.16. Typical LIF measurement of the argon IVDF, measured in LEIA, $126 \mathrm{~cm}$ downstream of the antenna (position D in Fig. 2.2). The black line is the raw LIF signal, while the red line is a single Gaussian fit to the data and the purple line is the iodine reference spectrum. This figure was obtained from Ref. [11].

\subsubsection{Re-entrant Probe}

The re-entrant probe that enters LEIA from the side of the vacuum chamber can be placed at a given location with a rotatable vacuum feedthrough. The rotatable feedthrough was constructed for use with Langmuir and LIF collection probes in LEIA (see Fig. 3.17). The 
feedthrough design was based on the schematics discussed in greater detail in Ref. [34]. The feedthrough was modified to use a QF-40 flange instead of the original design for QF-50 for compatibility with existing probe hardware at WVU. The ball, sealed with two O-rings, enables angular motion of the probe. Linear motion is provided by a double O-ring seal that is connected to the end of the ball by the QF-40.

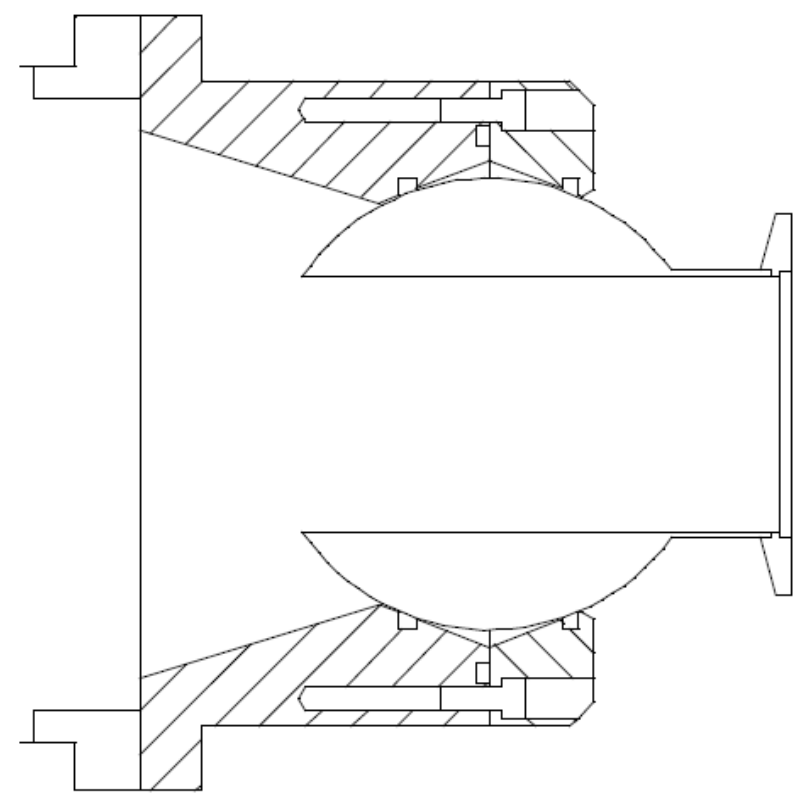

Figure 3.17. Mechanical drawing of the UCLA tilting port, modified for use at WVU. Figure is adapted from Ref. [32].

The design of the collection optics in the re-entrant probe is shown in Fig. 3.18. The probe contains miniature collection optics in a glass tube inserted into the plasma through the rotatable feedthrough. When the laser is injected from the HELIX end of the experiment, parallel to the axis of the chamber, the fluorescent emission is collected by the re-entrant probe to obtain parallel measurements of the IVDF in LEIA. A 1/4” plano-convex lens focuses the emission from 
the $461 \mathrm{~nm}$ line into the fiber. The fiber is then connected to the same PMT used for typical LIF measurements in HELIX chamber.

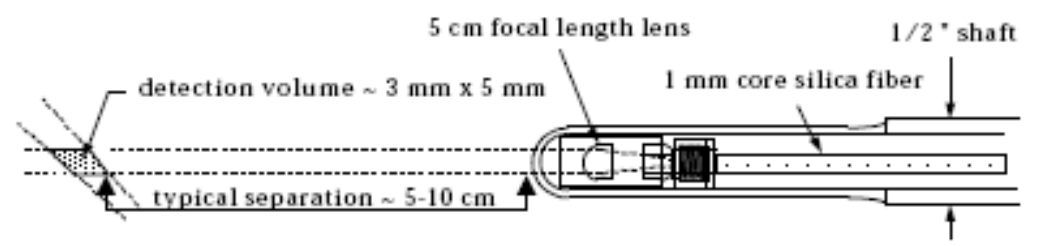

Figure 3.18. Schematic of the re-entrant probe. Figure adapted from Ref. [8].

\subsection{Time Resolved LIF}

An overview of time resolved LIF measurement techniques developed by the WVU HELIX group and others is provided in Ref. [11]. The greatest time resolution that can be achieved when using LIF is the lifetime of the optically pumped upper state. For the LIF scheme used for these experiments, the lifetime is a few nanoseconds. However several limiting factors prevent temporal investigations from achieving such time resolution. These limitations include the sampling speed of the data acquisition system, the RC time constants of the electronics, particular plasma conditions, and the ability to collect a sufficient number of LIF photons for a decent signal to noise ratio. The digital oscilloscope used here is the Tektronix TVS641 waveform analyzer. Typically, the sampling rate is set to $50 \mathrm{kHz}$. The biggest limiting factor comes from the need for a 1 ms integration time on the lock-in. As noted in previous studies by the WVU HELIX group, ${ }^{35}$ to get sufficient signal to noise, a combination of a high enough number of on-pulses for a given time constant of integration must be achieved. The acousto-optic modulator (AOM) was used in all time resolved LIF measurements instead of a mechanical 
chopper because it had the capability to deliver more pulses of laser light within a fixed time window. The mechanical chopper has an upper frequency limit of $1 \mathrm{kHz}$ while typical AOM measurements were performed at $40 \mathrm{kHz}$.

Another way to improve upon the signal to noise ratio with time resolved LIF is to average many measurements together. Similar to CW LIF, the laser frequency is fixed while a large number of LIF measurements are obtained for a single frequency step in the IVDF measurement. A typical time resolved LIF scan across a bimodal IVDF requires 50-75 frequency steps for good velocity resolution. Time resolved LIF experiments were performed in a pulsed plasma source. The pulsed operation of $5 \mathrm{~Hz}$ at a $50 \%$ duty cycle was accomplished by amplitude modulation of the driving frequency of the plasma antenna. The LIF system configuration used for time resolved LIF with a lock-in amplifier is shown in Fig. 3.19. A typical time resolved LIF measurement of a double layer for experimental parameters similar to those used in these experiments is shown in Fig. 3.20. ${ }^{36}$ 


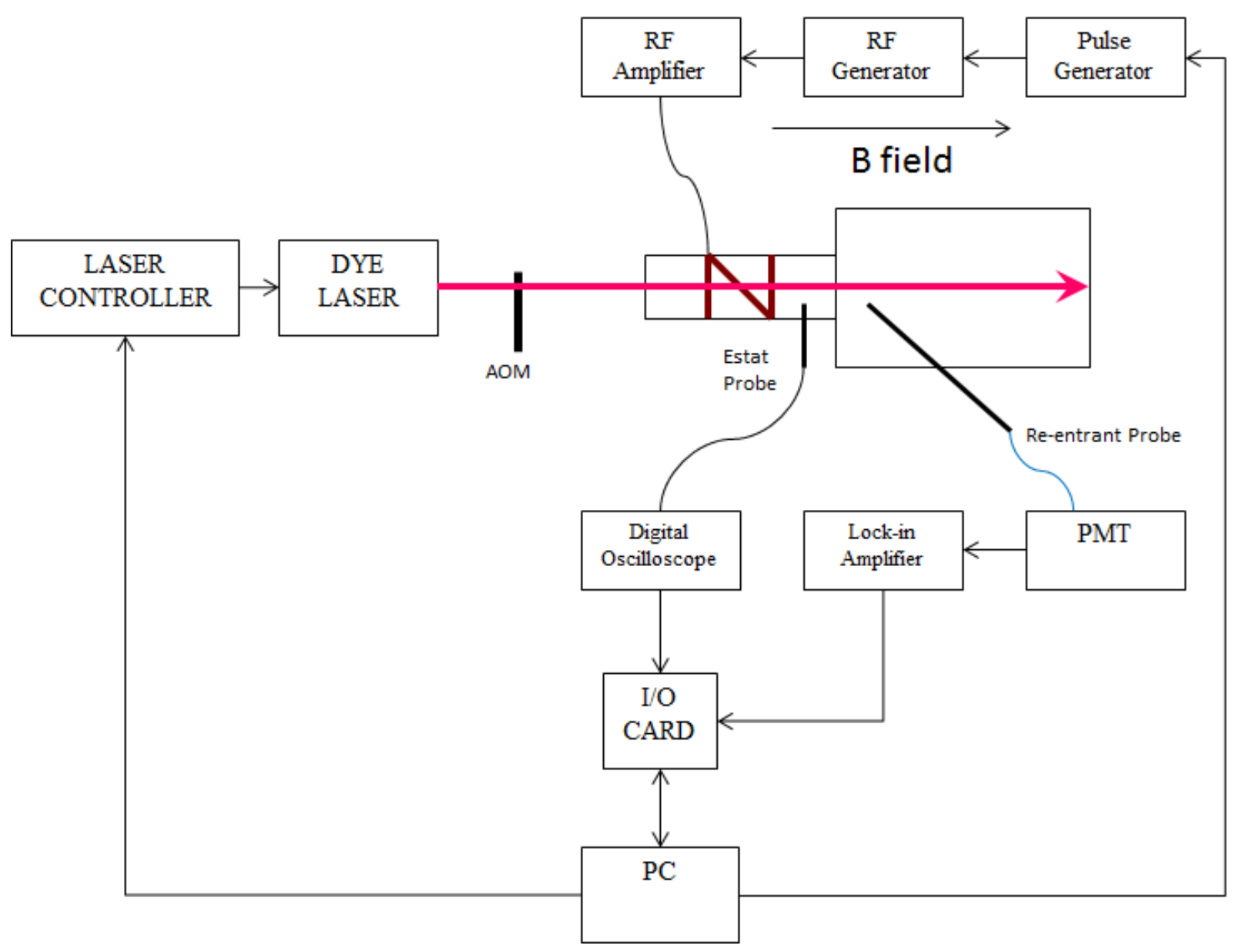

Figure 3.19. The time resolved LIF diagnostic configuration. The reference beam path is removed from the drawing for simplicity. 


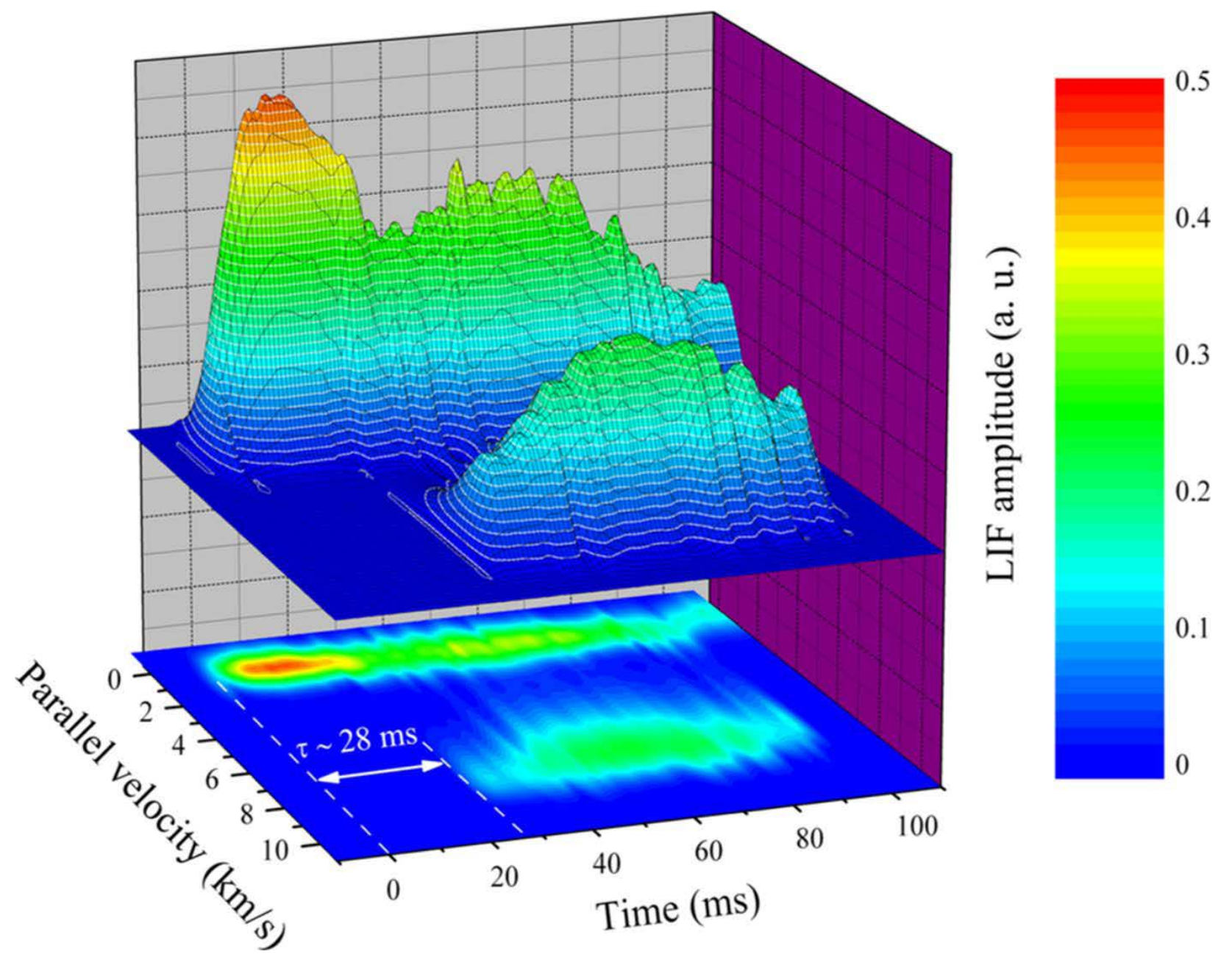

Figure 3.20. Example of a LIF-determined argon ion velocity distribution function during a $100 \mathrm{~ms}$ plasma pulse with $1 \mathrm{~ms}$ time resolution. The top portion is surface plot showing a fast $(\sim 7.1 \mathrm{~km} / \mathrm{s})$ and a slow $(\sim 0.4 \mathrm{~km} / \mathrm{s})$ ion population. The bottom portion is a contour plot showing the time lag of $\sim 28 \mathrm{~ms}$ in the appearance of the fast ion population. This figure was obtained from Ref. [36].

Correlation between two time series such as an LIF signal and an electrostatic fluctuation signal presents additional challenges due to the need for synchronous recording and identical sampling speeds. Either the two signals must be recorded on the same digitizer or there must be a time stamp system that can be used to align both time series to a common time base. For these experiments the LIF and electrostatic fluctuation signals were recorded with the same digitizer. 
A key point is that the lock-in amplifier introduces 1 ms time resolution, or an effective sampling speed of $1 \mathrm{kHz}$. This frequency limit prevents any correlation studies with electrostatic signals at frequencies above $1 \mathrm{kHz}$.

Higher frequency sampling is achieved by removal of the lock-in amplifier and AOM from the LIF system and recording the plasma emission for a fixed laser frequency during the plasma pulse. A large number of averages of the signal are recorded to reduce the noise arising from the spontaneous plasma emission at the fluorescent wavelength. The PMT signal and electrostatic probe signals were recorded with a LeCroy WaveRunner ${ }^{\mathrm{TM}} 604 \mathrm{Zi}$ oscilloscope. The WaveRunner has the capability of performing onboard averages of FFTs. For each plasma pulse the oscilloscope records both time series and then stores averages of the real and imaginary parts of the cross power spectra between the two signals. To investigate the correlation between the particular portions of the IVDF, e.g., the bulk and beam populations, and the electrostatic fluctuations, the average cross power spectrum was recorded for those laser frequencies at which peaks in the IVDF appeared during measurements in CW plasmas. 


\section{Chapter 3 References}

${ }^{1}$ I. Langmuir, Phys. Rev. 28, 727 (1926).

${ }^{2}$ F. F. Chen, in Plasma Diagnostic Techniques, ed. by R. H. Huddlestone and S. L. Leonard (Academic Press, New York, 1965).

${ }^{3}$ L. Schott, in Plasma Diagnostics, ed. by W. Lochte-Holtgreven (John Wiley, New York, 1968).

${ }^{4}$ I. H. Hutchinson, Principles of Plasma Diagnostics, (Cambridge University Press, 1987).

${ }^{5}$ N. Hershkowitz, How Langmuir Probes Work, in Plasma Diagnostics, Discharge Parameters and Chemistry, ed. by O.Aucillo and. D.L. Flamm (Academic Press, 1989).

${ }^{6}$ V. I. Demidov, S. V. Ratynskaia and K. Rypdal, Rev. Sci. Instrum. 73, 3409 (2002).

${ }^{7}$ T. E. Sheridan and J. Goree, Phys. Rev. E, 50, 2991 (1994)

${ }^{8}$ P. A. Keiter, E. E. Scime, M. M. Balkey, R. Boivin, J. L. Kline and S. P. Gary, Phys. Plasmas 7, 779 (2000).

${ }^{9}$ N. A. Krall and A. W. Trivelpiece, Principles of Plasma Physics, (San Francisco Press, 1986).

${ }^{10}$ J. C. Sprott, Electrostatic Probe Techniques, PLP 88, University of Wisconsin, Madison (1966).

${ }^{11}$ I. A. Biloiu, Laser Induced Fluorescence Studies of Ion Acceleration in Single and Multiple Species Expanding Plasmas, Ph. D. Thesis, West Virginia University, Morgantown (2009).

${ }^{12}$ I. D. Sudit and F. F. Chen, Plasma Sources Sci. Technol. 3, 162 (1994).

${ }^{13}$ A. M. Keesee, Neutral Density Profiles in Argon Helicon Plasmas, Ph. D. Thesis, West Virginia University, Morgantown (2005).

${ }^{14}$ P.A. Keiter, Experimental Investigation of Ion Temperature Anisotropy Driven Instabilities in a High Beta Plasma, Ph. D. Thesis, West Virginia University, Morgantown (1999). 
${ }^{15}$ M. J. Burin, G. R. Tynan, G. Y. Antar, N. A. Crocker and C. Holland, Phys. Plasmas 12, 052320 (2005).

${ }^{16}$ J. M. Beall, Y. C. Kim and E. J. Powers, J. Appl. Phys. 53, 3933 (1982).

${ }^{17}$ R. A. Hardin, Measurement of Short-Wavelength Electrostatic Fluctuations in a Helicon Plasma Source, Ph. D. Thesis, West Virginia University, Morgantown (2008).

${ }^{18}$ S. Chakraborty Thakur, Understanding Plasmas through Ion Velocity Distribution Function measurements, Ph. D. Thesis, West Virginia University, Morgantown (2010).

${ }^{19}$ C. Charles, A. W. Degeling, T. E. Sheridan, J. H. Harris, M. A. Leiberman and R. W. Boswell, Phys. Plasmas 7, 5232 (2000).

${ }^{20}$ Z. Harvey, S. Chakraborty Thakur, A. Hansen, R. A. Hardin, W. S. Przybysz and E. E. Scime, Rev. Sci. Instrum. 79, 10F314 (2008).

${ }^{21}$ C. Bohm and J. Perrin, Rev. Sci. Instrum. 64, 31 (1993).

${ }^{22}$ F. Valsaque, G. Manfredi, J. P. Gunn and E. Gauthier, Phys. Plasmas 9, 1806 (2002).

${ }^{23}$ W.J. Miloch, N. Gulbrandsen, L.N. Mishra, and Å. Fredriksen, Appl. Phys. Lett. 97, 261501 (2010).

${ }^{24}$ W.J. Miloch, N. Gulbrandsen, L.N. Mishra, and Å. Fredriksen, Phys. Plasmas 18, 083502 (2011).

${ }^{25}$ N. Gulbrandsen, W. J. Miloch, and Å. Fredriksen, Contrib. Plasma Phys. 53, No. 1, 27 (2013).

${ }^{26}$ J. L. Kline, Slow Wave Ion Heating and Parametric Instabilities in the HELIX Helicon Source, Ph. D. Thesis, West Virginia University, Morgantown (2002).

${ }^{27}$ Y.T. Yardley and C. B. Moore, Journal of Chemical Physics 45, 1066 (1966).

${ }^{28}$ R. A. Stern and J. A. Johnson, Phys. Rev. Lett. 34, 1548 (1975).

${ }^{29}$ D. N. Hill, S. Fornaca and M. G. Wikham, Rev. Sci. Instrum. 54, 309 (1983). 
${ }^{30}$ R. F. Boivin, Line Broadening Mechanisms, PL-039, West Virginia University, Morgantown (1999).

${ }^{31}$ R. F. Boivin, Zeeman Splitting for LIF Transitions and De-convolution Technique to Extract Ion Temperatures, PL-050, West Virginia University, Morgantown (2002).

${ }^{32}$ X. Sun, A Study of Ion Acceleration, Asymmetric Optical Pumping and Low Frequency Waves in Two Expanding Helicon Plasmas, Ph. D. Thesis, West Virginia University, Morgantown (2005).

${ }^{33}$ H. Salami and A. Ross, J. Molec. Spectr. 233, 157 (2005).

${ }^{34}$ D. Leneman and W. Gekelman, Rev. Sci. Instrum. 72, 3473 (2001).

${ }^{35}$ I. A. Biloiu and E. E. Scime, Appl. Phys. Lett. 95, 051504 (2009).

${ }^{36}$ I. A. Biloiu and E. E. Scime, IEEE Trans. Plasma Sci. 36, 1376 (2008). 


\section{Chapter 4: Signal Processing Techniques}

As noted earlier, an instability with properties consistent with those of an ion acoustic instability was observed whenever formation of the double layer was suppressed in previous steady-state HELIX experiments. ${ }^{1}$ The characteristics of the instability were determined through analysis of the frequency spectra of electrostatic waves in the plasma. In this Chapter, the essential aspects of those earlier studies are reviewed and described to place the time-resolved, spectral measurements presented in this work in context and to justify the need to introduce analysis methods capable of generating time resolved frequency spectra.

A common analogy used in the signal processing literature to describe the inadequacy of having only one piece of information, i.e., a frequency spectrum, to analyze a time series measurement is based on the way human beings hear music. ${ }^{2}$ Let a musical score represent a time series. Each note in the score conveys four pieces of information: frequency (vertical location on the score), position in time (horizontal location), duration (tempo and the type of note), and intensity (denoted by accent, crescendo, decrescendo, etc.). Omitting three of these four parameters, i.e., keeping only one descriptive characteristic of the score, makes the music unrecognizable. At the beginning of every score, global information such as “C major” is given. The dominant tones (major peaks in the power spectrum) within the piece of music make up chords that define the major and minor scales. Since many pieces of music share this $\mathrm{C}$ major characteristic, a musical score cannot be identified by this one feature. Time-resolved information, i.e., what frequencies occur at what times, is needed to uniquely identify a musical composition. By itself, a frequency spectrum of a time series measurement does not fully 
describe the time series measurement - particularly if some of the signals embedded in the time series are not stationary.

The time-frequency analysis techniques described in this chapter provide a means of recovering time resolved frequency spectra information (what frequencies occur at what times) from time series measurements. The discussion begins with brief review of the short time Fourier transform (STFT) method, which is a natural extension of the conventional Fourier analysis method used to extract the frequency spectrum of an entire time series. The STFT method delivers coarse-grained time and frequency information for a single time series. The wavelet transform, discussed next, is an adaptive time-frequency analysis method that optimizes the time resolution information for each frequency embedded in a time series. The strengths and weaknesses of the different signals processing techniques are demonstrated through application to measured time series. The most appropriate analysis methods are then employed in the timeresolved double layer studies in the HELIX-LEIA system described in Chapter 5.

\subsection{The Ion Acoustic Instability and Double Layer Formation}

In 2009, Chakraborty Thakur et al. demonstrated that slight variations in the coupling of rf power into a helicon source could, for constant magnetic field strengths and neutral pressure, affect the strength of a spontaneously forming double layer in an expanding helicon source

plasma. ${ }^{1}$ Chakraborty Thakur et al. observed that as the rf coupling improved and the potential drop across the double layer increased, i.e., the double layer grew stronger, a threshold was reached and the double layer vanished. The rf coupling was varied in those experiments by changing the antenna driving frequency. At lower driving frequencies the coupling was better 
than at higher driving frequencies. The threshold for double layer formation appeared at driving frequencies between 11 and $12 \mathrm{MHz}$. Below $11.5 \mathrm{MHz}$, the double layer (DL) vanished. At the same time the double layer vanished, it was observed that Langmuir probe measurements were extraordinarily noisy. Each measurement had to be repeated many times to obtain reliable average density and electron temperature values. Above source frequencies of $12 \mathrm{MHz}$, a single measurement was sufficient. ${ }^{3}$ Shown in Fig. 1 are multi-tip probe measurements of the power spectra of the upstream electric field fluctuations versus driving frequency. For driving frequencies below $11.5 \mathrm{MHz}$, the spectra are dominated by a wave at a fundamental frequency of $17.5 \mathrm{kHz}$ and its harmonics. Coincident with the appearance of the current-free DL, there is a dramatic reduction in the amplitude of the floating potential fluctuations both upstream and downstream of the expansion region. From phase measurements between the probe tips, it was determined that the $17.5 \mathrm{kHz}$ wave propagates primarily in the axial direction with a wavelength of approximately $3 \mathrm{~cm}$. 


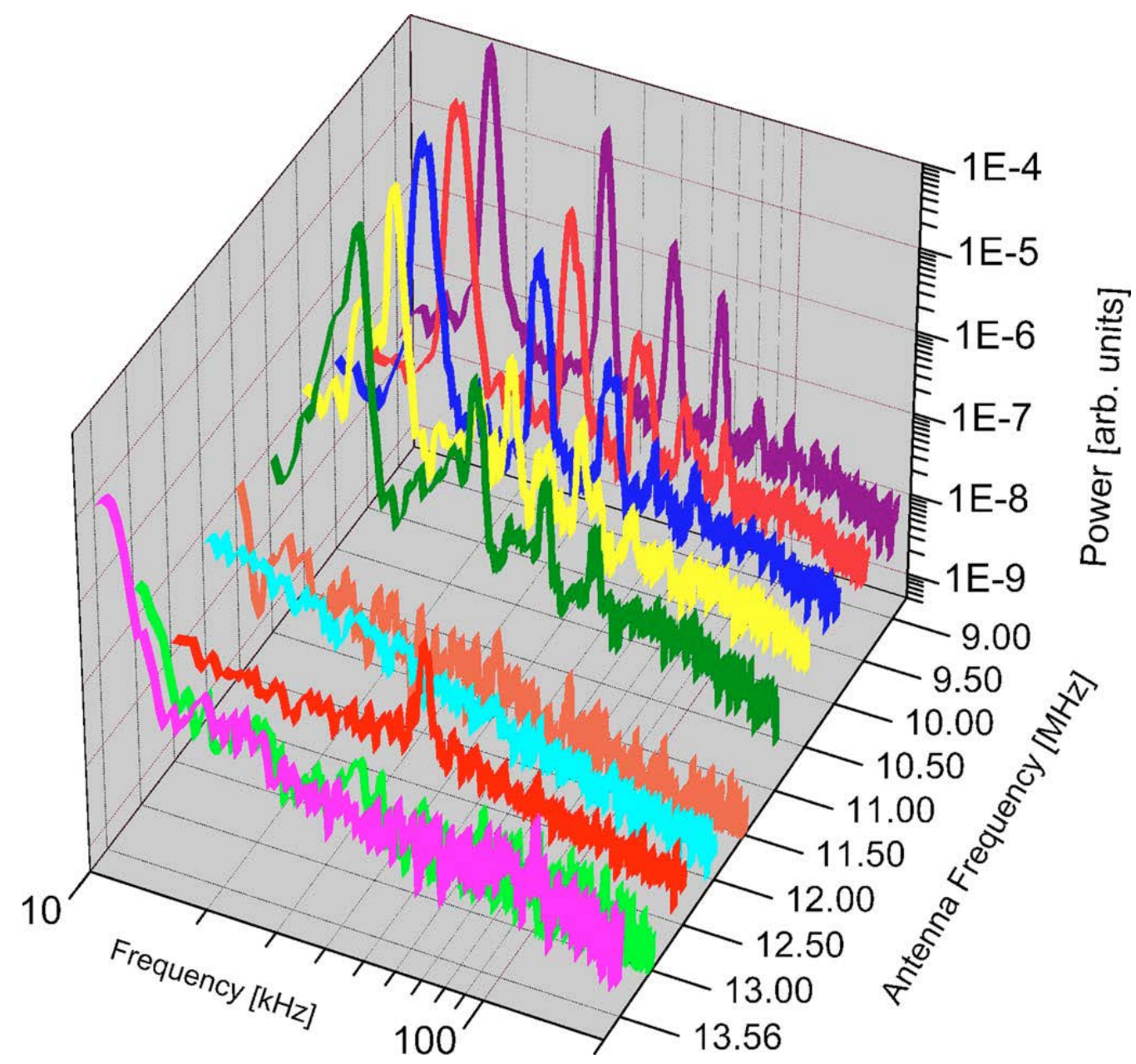

Figure 4.1. The power spectrum of the electrostatic instabilities versus antenna frequency obtained $50 \mathrm{~cm}$ downstream of the antenna. The instability at $17.5 \mathrm{kHz}$ and its harmonics appears only for the lower antenna frequencies. Figure obtained from Ref. [1].

The Bohm criterion for ions entering a sheath (or DL) is that their minimum speed be the Bohm sound speed, defined as $\sqrt{k_{B} T_{e} / m_{i}}$ for ions. ${ }^{4}$ We note that if the Bohm criterion for ions entering a sheath with speeds larger than the ion sound speed is satisfied for a DL, it is possible that current driven instabilities will be excited in the DL, e.g., ion acoustic and cyclotron instabilities. Since there are at least four different populations of particles, excitation of a twostream instability is also possible. Among these instabilities, the threshold for the Buneman 
instability yields the same current criterion as the Bohm criterion. ${ }^{1}$ Other instabilities, e.g., the Pierce instability, may trap ions in a narrow strongly varying potential and form a laminar DL. ${ }^{5}$ Once formed, the charge separation of the DL can persist without the presence of the Pierce instability since the charge distribution of a DL is one of the solutions of the Bhatnagar-GrossKrook (BGK) equation. Ion-acoustic driven DLs, characterized by an ion hole on the low potential side and a potential difference equal to or less than the electron temperature, ${ }^{6}$ are also observed to coincide with the existence of ion acoustic instabilities in computer simulations. ${ }^{7}$ Experimentally, ion cyclotron instabilities have been observed in a triple plasma (TP) device DL experiments in an inhomogeneous magnetic field. ${ }^{8}$

For observed waves, their axial propagation rules out drift waves as the source of the instabilities. The short parallel wavelength and multi-harmonic excitation are inconsistent with the characteristics of the ionization instability observed in other DL experiments. ${ }^{9}$ The multiple harmonics spanning frequencies below and above the ion cyclotron frequency are inconsistent with electrostatic ion cyclotron waves. Given that the wavelengths are much larger than the Debye length but much shorter than the system size, the fact that they propagate along the background magnetic field, and that excitation of the waves appears to be associated with a threshold particle drift velocity ion beam speed comparable to the ion sound speed, the waves are most likely due to a beam driven ion acoustic instability. ${ }^{10}$

The dispersion relation for the ion acoustic mode, for ion temperatures much smaller than the electron temperature, is

$$
\omega^{2}=\frac{1}{\left(1+\gamma \lambda_{D e}^{2} k^{2}\right)}\left(\frac{\gamma k_{B} T_{e}}{m_{i}}\right) k^{2}
$$


where $\omega$ is the wave frequency, $k$ is the wave number, $\lambda_{D e}$ is the electron Debye length, $\gamma$ is the ratio of the specific heats, $k_{B}$ is the Boltzmann constant, and $T_{e}$ is the electron temperature.

For large values of $k$, the frequency asymptotically approaches the ion plasma frequency. For frequencies much less than the ion plasma frequency ( $\sim 00 \mathrm{MHz}$, in this experiment) and for values of $k$ smaller than $1 / \lambda_{D e}$, $\left(12 \mathrm{~cm}^{-1}\right.$ for these experimental conditions) the wave frequency is linear in $k$ and the phase velocity is approximately given by the ion sound speed.

$$
\frac{\omega}{k}= \pm \sqrt{\frac{\gamma k_{B} T_{e}}{m_{i}}}
$$

For these experimental conditions, the ion sound speed is $6.4 \mathrm{~km} / \mathrm{s}$. The measured average phase velocity of the $17.5 \mathrm{kHz}$ wave is $7 \pm 1 \mathrm{~km} / \mathrm{s}$, which strongly suggests that these waves are ion acoustic waves.

In previous experiments, ${ }^{11}$ it was shown that formation of a DL in pulsed helicon source plasma occurs a few milliseconds after the initiation of the discharge (see Fig. 3.20 for an example of a time-resolved IVDF measurement of a beam and background ion population). The primary scientific objective of the experiments reported here is to determine if there is a causal relationship between the DL and the observed ion acoustic waves. In other words, does the appearance of the DL trigger the growth of the waves and if they waves grow large enough, can they force the collapse of the DL? To address the issue of a causal relationship, the complete IVDF and the plasma floating potential fluctuation amplitude were measured as a function of time throughout $100 \mathrm{~ms}$ long discharges pulsing at a repetition rate of $5 \mathrm{~Hz}$ with a $50 \%$ duty cycle. Through careful source tuning, plasmas were created at the same source parameters which 
yielded cases with and without DLs in steady-state. Because conventional Fourier analysis of the time series measurements of the plasma floating potential does not provide a measure of the evolution of instabilities in a pulsed plasma, new analysis methods were developed for frequency spectra measurements in pulsed plasmas.

\subsection{Fourier and Wavelet Transform Methods for Signal Processing}

Selecting the most appropriate signal processing technique requires a thorough understanding of the signal to be analyzed. For these discussions, the term "signal" refers to a measurement of a physical quantity. Signals are classified as deterministic or nondeterministic depending upon their reproducibility. Signals that are deterministic can be generated repeatedly with identical results; nondeterministic signals, i.e., random signals, cannot.

There are two types of deterministic signals: periodic and transient. Periodic signals repeat themselves exactly after a certain period of time (sinusoidal signals for example). Transient signals are defined as signals that appear for only a short period of time but can still be represented analytically. A good example of a transient signal source is a damped oscillator. Nondeterministic signals are generally described in statistical terms simply because they don’t explicitly follow mathematical expressions. There are two types of nondeterministic signals: stationary and non-stationary. The statistical properties of a stationary signal do not change with time while the statistical properties of a non-stationary signal changes with time.

Signal classification methods are not rigid or exclusive; no real signal is ever completely deterministic. A real signal may have several of the characteristics described above. However, 
even imperfect classification is extremely valuable for selection of the most appropriate signal processing method. $^{12}$

Typically, a signal processing technique transforms a time-domain signal into another domain, with the goal of extracting additional information embedded within a time series; information that is otherwise not readily observable in the original form of the time series. In general, signal processing techniques compare a time series measurement to a template function to determine their level of "similarity." The inner product of a signal with a well-chosen template function quantifies the degree of similarity. Mathematically, in the Lebesgue space $L^{2}(\mathbb{R})$, an inner product between two functions is

$$
\langle p, q\rangle \equiv \int_{-\infty}^{\infty} p(t) q^{*}(t) d t
$$

where ()$^{*}$ denotes the complex conjugate and $\mathbb{R}$ is the real number line. ${ }^{13}$ Usually, the comparison is made with a time domain signal, $x(t)$ and a set of template functions $\left\{\psi_{n}(t)\right\}_{n \in \mathbb{Z}}$ where $\mathbb{Z}$ is set of integers. The inner product of these two functions returns a set of coefficients that can be expressed (using Eq.4.3) as either ${ }^{12}$

$$
c_{k}=\int_{-\infty}^{\infty} x(t) \psi_{k}^{*}(t) d t
$$

or in the more general from,

$$
c_{k}=\left\langle x, \psi_{k}\right\rangle
$$


Like a scalar product between two vectors, the inner product between $x(t)$ and $\left\{\psi_{k}(t)\right\}_{k \in \mathbb{Z}}$ is greatest when the two functions are most similar. Both the Fourier transform and the wavelet transform take advantage of inner products to quantify the degree of similarity.

\subsubsection{The Fourier Transform}

The Fourier transform is probably the most widely used signal processing tool in engineering and science. The Fourier transform takes a continuous time series $(x(t))$ in the time domain and delivers the frequency composition of the signal by transforming it into the frequency domain. Jean Baptiste Joseph Fourier, a French mathematician, discovered the principles behind this relationship in 1807. Objections from some of his contemporaries such as Joseph-Louis Lagrange kept Fourier from publishing this finding until 1822 in his book, The Analytic Theory of Heat.

Expressed in the inner product form presented in Eq. 4.3, the Fourier Transform is

$$
X(v)=\left\langle x, e^{i 2 \pi v t}\right\rangle=\int_{-\infty}^{\infty} x(t) e^{-i 2 \pi v t} d t
$$

where $v$ is the frequency of a purely sinusoidal template function. Eq. 4.6 assumes that the signal has finite energy, $\varepsilon$. The energy of a signal, $x(t)$, is defined as the integral of the squares of all the signal's values. In order for there to be an inverse Fourier transform, the energy of a signal must converge ${ }^{14}$

$$
\varepsilon=\int_{-\infty}^{\infty}|x(t)|^{2} d t<\infty
$$


The term "energy" is used because the sum of squares is a common occurrence in physics when various types of energy are calculated. ${ }^{15}$ The inverse of the Fourier transform, to take a signal from the frequency domain to the time domain, is

$$
x(t)=\int_{-\infty}^{\infty} X(v) e^{i 2 \pi v t} d v
$$

Note that Eq. 4.7 necessitates the knowledge of the complete history of the signal from $-\infty$ to $\infty$ in order to generate one frequency. Conversely, Eq. 4.8 states that each value of $x(t)$ at one instant, $t$, can be thought of as an infinite superposition of complex exponentials, i.e. infinite non local waves. Without going any further, it is very apparent that Fourier transforms are well suited for deterministic, periodic signals and not transient or nonstationary signals.

Equations 4.7 and 4.8 are intended for use with continuous signals. However, real signals are acquired through discrete sampling over a finite interval at discrete time intervals, $\Delta T$, over a total measurements time $T$. Fourier analysis of discrete signals is accomplished through the discrete Fourier transform (DFT)

$$
\operatorname{DFT}\left\{x_{n}\right\}=\hat{x}_{k}=\frac{1}{N} \sum_{n=0}^{N-1} x_{n} e^{-i 2 \pi v_{k} n \Delta T}
$$


where $N=\frac{T}{\Delta T}$ is the number of samples, $n$ is the sample index, $x_{n}$ is the $n^{\text {th }}$ sample of a discretely sampled physical quantity, and $v_{k}=\frac{n}{T}, k=0,1,2, \ldots, N-1$ are the discrete frequency components. The inverse DFT is ${ }^{12}$

$$
D F T^{-1}\left\{\hat{x}_{k}\right\}=x_{n}=\frac{1}{\Delta T} \sum_{v_{k}=0}^{(N-1) / T} \hat{x}_{k} e^{i 2 \pi v_{k} n \Delta T}
$$

Calculating the DFT of a real signal is computationally intensive. The DFT of $N$ samples requires the multiplication of a $N \times N$ matrix and the number of calculation steps is on the order of $N^{2}$. Thus, considerable computational cost results from improving the time resolution of a measurement that is to be Fourier analyzed. DFT analysis of signals in science and engineering was rare until the Cooley-Tukey algorithm provided a computationally efficient way to implement the DFT in 1965. The Cooley and Tukey algorithm is a variant of what is now known as a Fast Fourier Transform (FFT). Other versions of the FFT existed as long ago as 1805, when Carl Friedrich Gauss created a version of the FFT that predated the work done by Fourier by 2 years. ${ }^{16}$ However, the Cooley-Tukey FFT (simply referred to as the FFT) has become the dominant FFT technique due to its need for fewer operations, reducing the number of calculations to $N \log (N)$. The FFT is a recursive algorithm and is easily implemented computationally.

As mentioned previously in Section 3.2.3, the Nyquist frequency serves as an upper limit of resolvable frequencies with the issue of aliasing. The lowest frequency that can be resolved with an FFT, $\Delta v$, is given by 


$$
\Delta v=\frac{v_{s}}{N}=\frac{1}{T},
$$

where $v_{s}$ is the sampling frequency. Note that the frequency resolution is determined solely by the acquisition time, a limit that will be discussed later.

As noted earlier with the continuous Fourier transform, it is the amplitude of the complex coefficients of each frequency component that quantifies how "much" of a given frequency is “in” the measured signal. The unnormalized power spectrum

$$
S_{x_{n} x_{n}}\left(\omega_{k}\right)=F F T\left\{x_{n}\right\} * F F T^{*}\left\{x_{n}\right\}
$$

is simply the amplitude of each discrete frequency component written in a mathematically compact form. The power spectrum Fig 4.1 is a good example of power of the FFT technique. The peaks in Fig. 4.1 clearly show what frequencies are present within each of the measured time series, an analysis that is impossible to perform in the time domain if more than one frequency component contributes to the time series. Although Fig 4.1 shows the presence of a $17.5 \mathrm{kHz}$ wave and harmonics when the antenna frequency was lower than $11 \mathrm{MHz}$, there is no way to determine from the FFT analysis when the wave activity appears within the time series. Two methods for deriving time-frequency domain information from a single time series are short-time Fourier transforms and wavelet transforms. 


\subsubsection{The Short-Time Fourier Transform}

The short-time Fourier transform (STFT) was created to address the temporal resolution limitations of the Fourier transform. The basis functions in the Fourier transform extend over an infinite time period, thus all information about the time dependence of any Fourier frequency component amplitude and phase are lost in the Fourier transform process. Dennis Gabor was the first to write about the STFT in 1946. The STFT uses a time localized Fourier transform within a sliding window. Using the inner product notation, the STFT is

$$
\begin{aligned}
\operatorname{STFT}(\tau, v)= & \left\langle x, w_{\tau, v}\right\rangle=\int x(t) w_{\tau, v}^{*}(t) d t \\
& =\int x(t) w(t-\tau) e^{-i 2 \pi v t}
\end{aligned}
$$

where $\tau$ is the moment in time where the sliding window function is centered, and $w(t)$ is the sliding window function. As Eq. 4.13 is advanced along the timeline, additional time-localized Fourier transforms are performed. The consecutive sliding window Fourier transforms repeats the process on the entire time series to be processed. Fig 4.2 shows a schematic for the STFT process. The STFT obtains the largest values at particular frequencies where the sliding window overlaps with intervals with the large contributions to the time series by components with that frequency. 


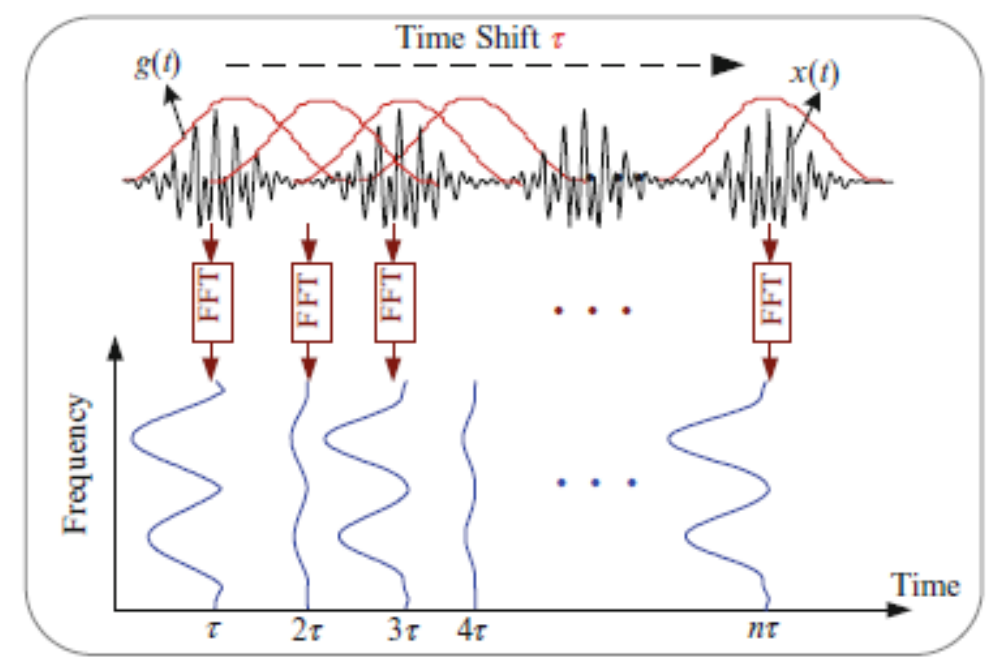

Figure 4.2. Illustration of short-time Fourier transform (STFT) on a test signal, $x(\mathrm{t})$. Figure obtained from Ref. [12].

The discrete version of the STFT used for discrete time series measurements is: ${ }^{17}$

$$
\begin{gathered}
\operatorname{STFT}(m, \omega)=\operatorname{DFT}(m, \omega)=\sum_{n=-\infty}^{\infty} x(n) w(n-m) e^{-i \omega n}, \\
0 \leq \omega<2 \pi,
\end{gathered}
$$

where $\omega$ is the frequency of the sinusoidal template function. Since Eq. 4.13 is a discrete convolution, it can be rewritten as

$$
\begin{gathered}
\operatorname{STFT}(m, \omega)=\operatorname{DFT}(m, \omega)=\sum_{n=-\infty}^{\infty} x(n-m) w(m) e^{-i \omega n} \\
0 \leq \omega<2 \pi
\end{gathered}
$$


by the commutative property. The analysis described in Section 5.2 uses a technique similar to Eq. 4.15 cross power spectra calculated for a series of discrete time windows. The time resolved cross power spectrum presented in Section 5.2 used a window width of 10 ms centered at $5 \mathrm{~ms}$ steps, e.g., 5, 10, 15...90, 95, throughout the 100 ms pulse.

Although STFTs enable Fourier analysis to provide some time-frequency information, STFTs do have some significant limitations. As with all discrete and bounded functions, the time resolution, $\Delta t$, and frequency resolution, $\Delta \omega$, are inextricably linked. The Heisenberg-Gabor uncertainty principle sets the lowest limit of the product of the two resolutions to be ${ }^{18}$

$$
\Delta t \Delta \omega \geq \frac{1}{2}
$$

Fig. 4.3 shows a diagram of how the resolution limits of the STFT method impacts the information that can be extracted from a time series measurement. ${ }^{2}$ The boxes drawn in Fig. 4.3a show that for a given window function, the time and frequency resolutions over the entire timefrequency plane are fixed. The shaded portion represents the results of the STFT analysis in the time-frequency domain. If the shaded box is lowered by one grid point, a longer width window in time would improve the technique’s capacity to identify lower frequency signals. Conversely, if the STFT is shifted upwards by one grid point, a smaller time window would be sufficient to distinguish between different high frequency elements. 

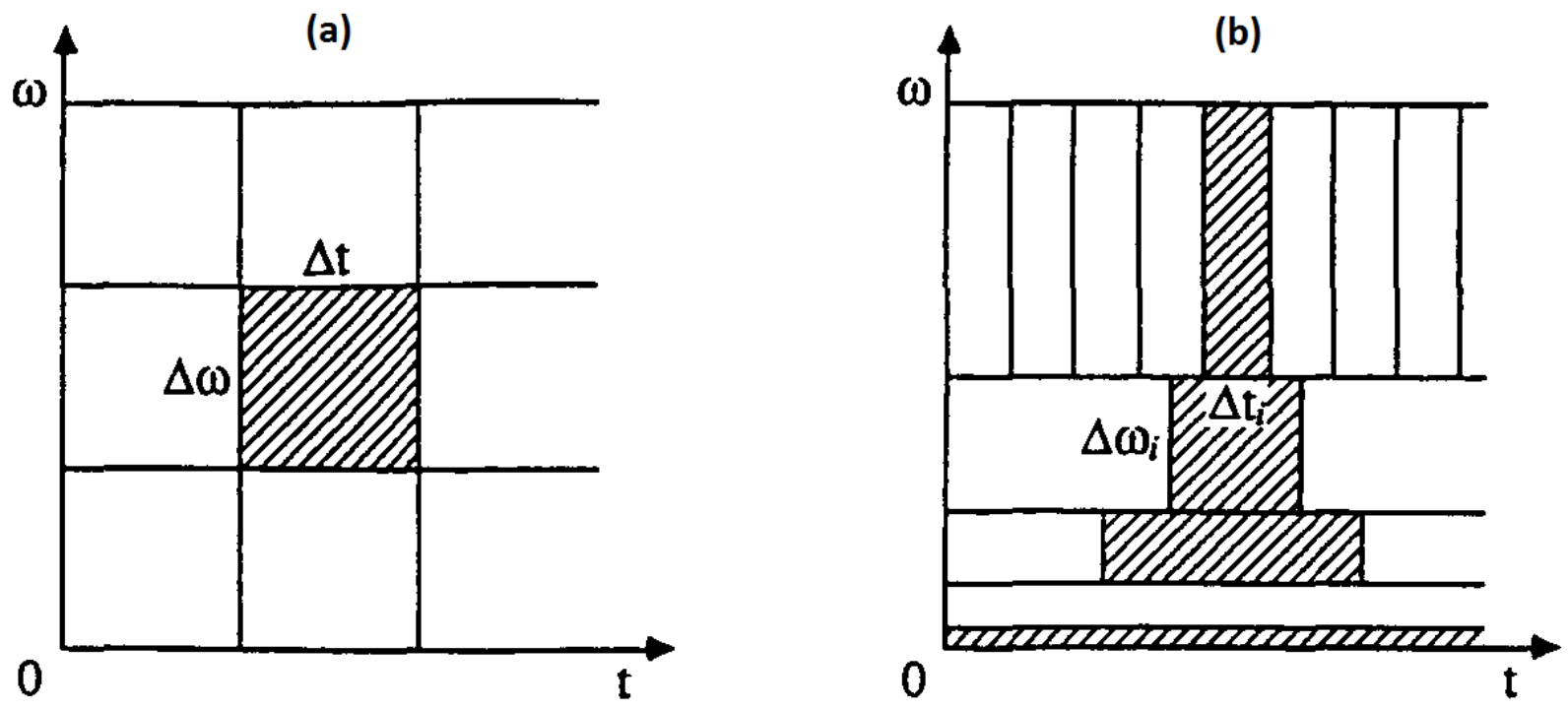

Figure 4.3. Time-frequency windows used in (a) a STFT and (b) a wavelet transform. Figure adapted from Ref. [2].

Various shapes of window functions have been developed over the past few decades, each of them specifically tailored for a particular type of signal. The Gaussian window, first used by Gabor, was designed for analyzing transient signals. Hamming and Hann windows are applicable to narrowband random signals, while the Kaiser-Bessel window is better suited for separating two signal components with frequencies very close to each other but with very different amplitudes. The choice of the window function directly affects the time and frequency resolution of the STFT analysis. ${ }^{12}$

The Hamming window was chosen for the experiments presented here because of the need for random signal detection while maintaining good frequency resolution. ${ }^{17}$ The formula for the Hamming window is

$$
w(n)=0.54+0.46 \cos \left(\frac{2 \pi n}{N}\right) .
$$


The weaknesses of the Hamming window are that it only has a "fair" ability to combat spectral leakage and does not generate particularly accurate spectral component amplitudes. Both of these weaknesses are somewhat ameliorated in this work by averaging over a large number of power spectra generated by STFT analyses of independent time series measurements.

Although STFTs allow Fourier analysis to provide some time-frequency information, the fixed frequency resolution for all center frequency bands is a significant limitation. Fig 4.3b shows how the wavelet approach combats the inflexibility of a STFT by using local base functions that are stretched and translated with a flexible resolution in both frequency and time. The wavelet transform is essentially a generalized version of the STFT.

\subsubsection{The Wavelet Transform}

The first publication of what is now called a “wavelet” occurred in 1909 in Alfred Haar’s dissertation, where he created a set of rectangular basis functions. However the idea of stretching and squeezing the window function along with the name "wavelet" surfaced in the mid 70's through the work of Jean Morlet and the team at the Marseille Theoretical Physics Center working under Alex Grossmann in France. The biggest advancement in the field occurred when Stéphane Mallat collaborated with Yves Meyer in 1988 to develop multi-resolution analysis (MRA) for wavelets. MRA made it possible to combine wavelet theory with the power of the fast discrete signals processing techniques found in engineering. MRA also gave researchers a mathematical framework to create their own base wavelets. Scientists such as Ingrid Daubechies were able to add new wavelet families and make other significant contributions to the field. ${ }^{19}$ Their work triggered the proliferation of wavelets into a myriad of disciplines such as audio and image processing (compression, denoising and detection) ${ }^{15}$ while providing a new signal analysis 
tool for manufacturing, ${ }^{12}$ understanding climate data, ${ }^{2,20,21}$ astronomy, biology and medicine. ${ }^{19}$ The first published account of the use of wavelets to study a laboratory plasma was by van Milligen et al. in $1995 .^{22}$ By combining wavelet analysis with bicoherence, they were able to measure structure in a turbulent plasma. More recent papers in plasma physics continue to employ the continuous wavelet transform because of its ability to detect intermittent events in the time-frequency domain. ${ }^{23,24,25,26}$

Similar to a short time Fourier transform, a continuous wavelet transform, $W$, is expressed as an inner product using Eq. 4.4 and 4.5 .

$$
W(s, \tau)=\left\langle x, \psi_{s, \tau}\right\rangle=\int_{-\infty}^{\infty} x(t) \psi_{s, \tau}^{*}\left(\frac{t-\tau}{s}\right) d t
$$

where $\tau$ represents the translation position, $s$ is the scale parameter that controls the dilation and $\psi_{s, \tau}$ is the "daughter wavelet" or simply "wavelet” and is derived from

$$
\psi_{s, \tau}\left(\frac{t-\tau}{s}\right)=\frac{1}{\sqrt{s}} \psi\left(\frac{t-\tau}{s}\right), \quad s>0, \tau \in \mathbb{R}
$$

where $\psi$ is the "mother wavelet,” “analyzing wavelet,” or "wavelet function.” Substituting Eq. 4.19 into Eq. 4.18 yields

$$
W(s, \tau)=\frac{1}{\sqrt{s}} \int_{-\infty}^{\infty} x(t) \psi^{*}\left(\frac{t-\tau}{s}\right) d t
$$


The scale parameter $s$, is inversely proportional to the signal's local frequency and depends on the properties of the mother wavelet. ${ }^{2,27}$

A wavelet is a waveform of finite duration with a mean of zero. These two requirements come from the admissibility condition, which insures mother wavelets are well localized and oscillate. ${ }^{28}$ Fig. 4.4 provides a visual comparison ${ }^{12}$ between a sine wave and a wavelet, in this case a Daubechies 4.

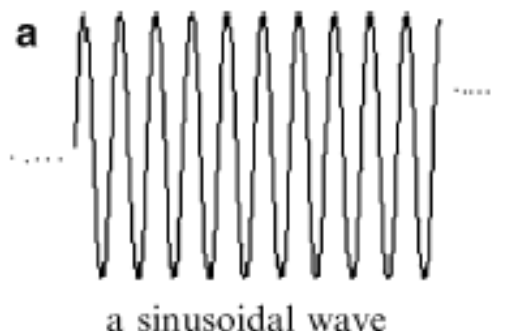

a sinusoidal wave b

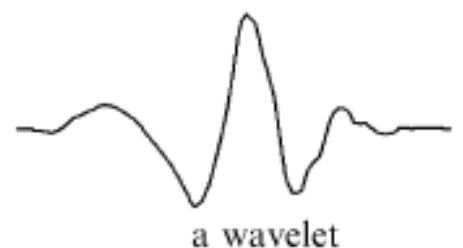

Figure 4.4. Representation of (a) a sine wave and (b) a Daubechies 4 wavelet. Figure obtained from Ref. [12].

The $s^{-1 / 2}$ normalizing factor in Eq. 4.20 ensures that every wavelet has the same unit energy as it undergoes the dilation process. This allows the wavelet transforms to be comparable at each scale. Fig 4.5 provides an illustration of the translation and dilation process outlined by Eq. 4.20, with Fig 4.5a being the original mother wavelet centered at $t=0$, Fig $4.5 \mathrm{~b}$ shows the mother wavelet undergoing translation, Fig. 4.5c shows the mother wavelet undergoing dilation, and Fig. 4.5d showing the mother wavelet undergoing translation and dilation. 


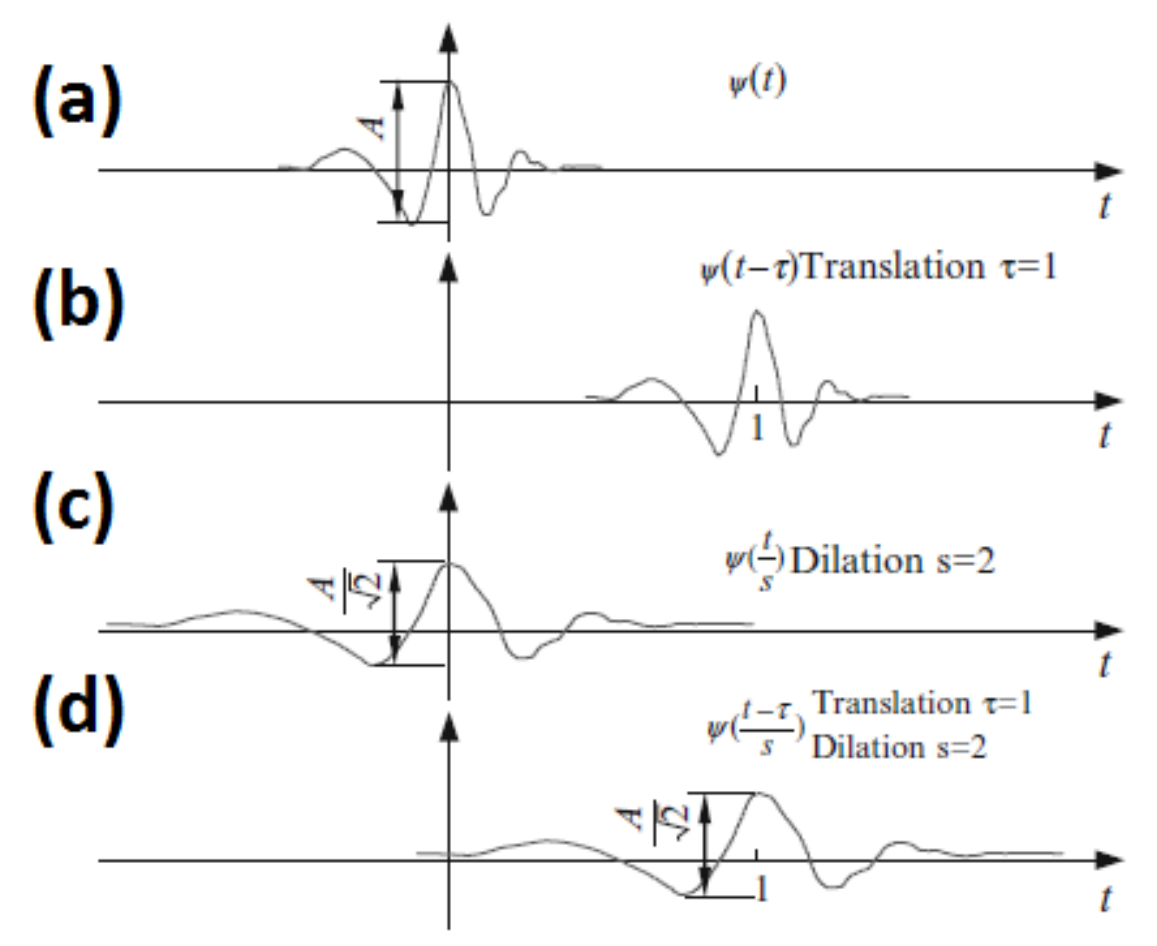

Figure 4.5. Illustration of translation (by the time constant $\tau$ ) and dilation (by the scaling factor $s$ ) while maintaining constant energy. (a) The original mother wavelet centered at $t=0$, (b) The mother wavelet undergoing translation, (c) The mother wavelet undergoing dilation, and (d) The mother wavelet undergoing translation and dilation. Figure adapted from Ref. [12].

\subsubsection{Implementation Methodology}

Daubechies outlines two classes of wavelet transforms in her book, Ten Lectures on Wavelets: the continuous wavelet transform and the discrete wavelet transform. ${ }^{29}$ The discrete wavelet transforms are further divided into orthonormal and non-orthonormal categories. Orthonormal discrete wavelets transforms used in MRA are better suited for data compression and reconstruction because of the compact support (efficient way of storing a signal's energy) they provide. Continuous wavelet transforms are not orthonormal and consequently are not as 
suited for compression. However, continuous wavelet transforms vary $s$ and $\tau$ continuously, unlike their discrete wavelet transform counterparts, making them better suited for capturing smooth variations in a time series. For these reasons, only continuous wavelet analysis is used in the analysis of the experiments described in this work. Along with the many articles written on the subject, the textbooks by Walker, ${ }^{15}$ Strang and Nguyen ${ }^{30}$ cover discrete wavelet transforms from very valuable perspectives and in greater detail.

Two approaches are used to obtain the continuous wavelet transform of a signal. One method is to obtain the wavelet coefficients, $\{W(s, \tau)\}$, analytically with the application of Eq. 4.17. The algorithm works as follows: Start with a wavelet and calculate the inner product of the signal and the wavelet for the first time interval of the measurement. Shift the wavelet by $\tau$ and repeat the inner product calculation. The time shifts and corresponding inner product calculations continue until the end of the time series is reached. At that point, the scale of the wavelet is adjusted by a given value and the above procedure is repeated for all of the scales. ${ }^{12}$

The second approach uses the convolution theorem and FFTs to complete the calculation. The convolution theorem in discrete form states that for two functions in the time domain, $f$ and $g$ and their convolution, $f * g$,

$$
\{f * g\}=D F T^{-1}(D F T\{f\} D F T\{g\}) .
$$

The continuous wavelet transform of a discrete sequence $x_{n}$ is defined as the convolution between $x_{n}$ and the discrete version of the wavelet, $\psi$ 


$$
W_{n}(s)=\sum_{n^{\prime}=0}^{N-1} x_{n^{\prime}} \psi^{*}\left[\frac{\left(n^{\prime}-n\right) \delta t}{s}\right]
$$

where $n, N$, and $\delta t$ are the time index, total number of samples, and the data time step, respectively. ${ }^{24}$ Since $W_{n}$ is the result of a convolution,

$$
W_{n}(s)=\sum_{k=0}^{N-1} \hat{x}_{k} \hat{\psi}^{*}\left(s \omega_{k}\right) e^{i \omega_{k} n \delta t}
$$

where $\hat{\psi}$ is Fourier transform of the wavelet function and where the angular frequency is given by

$$
\omega_{k}=\left\{\begin{array}{c}
\frac{2 \pi k}{N \delta t}: k \leq \frac{N}{2} \\
-\frac{2 \pi k}{N \delta t}: k>\frac{N}{2}
\end{array}\right\} .
$$

FFTs allow calculations of the continuous wavelet transform to be performed very efficiently and this method is used to numerically calculate the wavelet transforms by computer. ${ }^{20}$ However, the analytic approach of Eq. 4.20 is used to describe the analysis methodology because of its relative simplicity.

The wavelet power spectrum is calculated from

$$
S(s, \tau)=W(s, \tau) W^{*}(s, \tau)
$$

and the wavelet phase is 


$$
\phi_{n}(s, \tau)=\tan ^{-1} \frac{\operatorname{Im}[W(s, \tau)]}{\operatorname{Re}[W(s, \tau)]}
$$

\subsubsection{Wavelet Function Selection}

Along with orthogonality, there are additional factors to consider when selecting a mother wavelet for the continuous wavelet transform.

A) Real or Complex: Wavelet functions can be either. Complex wavelet functions are better at capturing oscillatory behavior and also provide phase information. Real wavelet functions are useful for isolating discontinuities and peaks.

B) Width: Like the STFT, a wavelet function's resolution is determined by the balance between its width in Fourier space and its width in the time domain thanks to the Heisenberg-Gabor uncertainty principle (Eq. 4.16). The larger the width in either domains, the more resolution in that domain.

C) Shape: The wavelet transform is an inner product, so the greater the similarity between the wavelet and the localized portion of signal it acts on, the greater chance for detection of an event.

With the consideration of these factors, the complex Morlet wavelet was chosen for the plasma fluctuation investigations presented in this work. The Morlet wavelet is smooth, continuous, well adapted to plasma fluctuations and non-stationary signals. As one of the more commonly used wavelets for time series analysis, the Morlet wavelet has proven its efficiency in other plasma fluctuation investigations. ${ }^{23,24,25,26,31}$ 
The Morlet wavelet is constructed by taking a plane wave and adding a Gaussian modulation

$$
\psi(\eta)=\pi^{-1 / 4} e^{i \omega_{o}} e^{-\eta^{2} / 2}
$$

where $\eta$ is the dimensionless time parameter and $\omega_{o}$ is the dimensionless frequency parameter. Fig 4.6 shows four mother wavelets for the purposes of comparison in the time domain and the frequency domain. ${ }^{20}$ On the left side of the figure, the time domain plots, the dashed lines represent the imaginary parts and the solid lines represent the real parts of the wavelets. Fig. 4(a) shows a Morlet wavelet for a dimensionless frequency of $\omega_{o}=6$. Like the Morlet wavelet, the Paul wavelet is also complex, while the derivatives of a Gaussian (DOG) are real. 
$\psi(\mathrm{t} / \mathrm{s})$
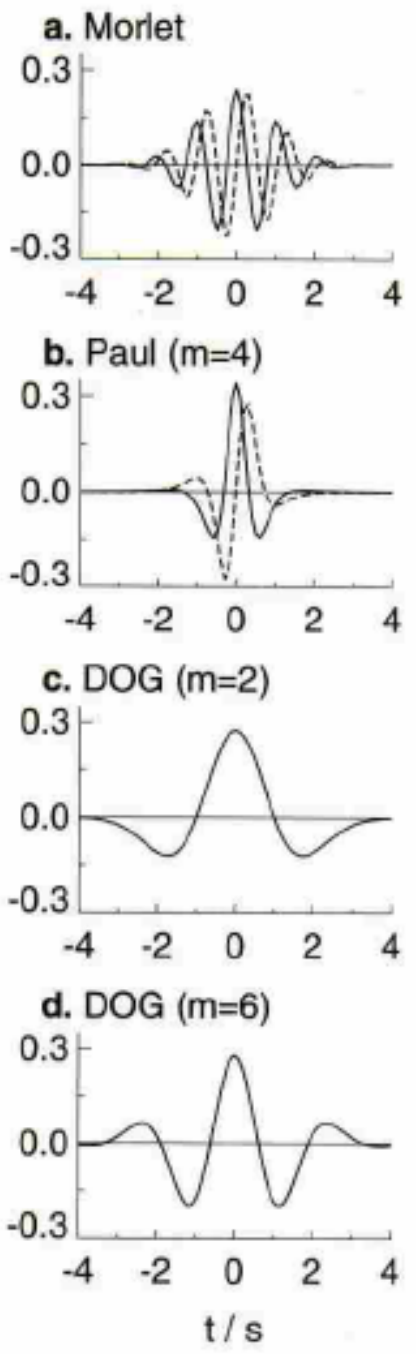

$\hat{\psi}(s \omega)$
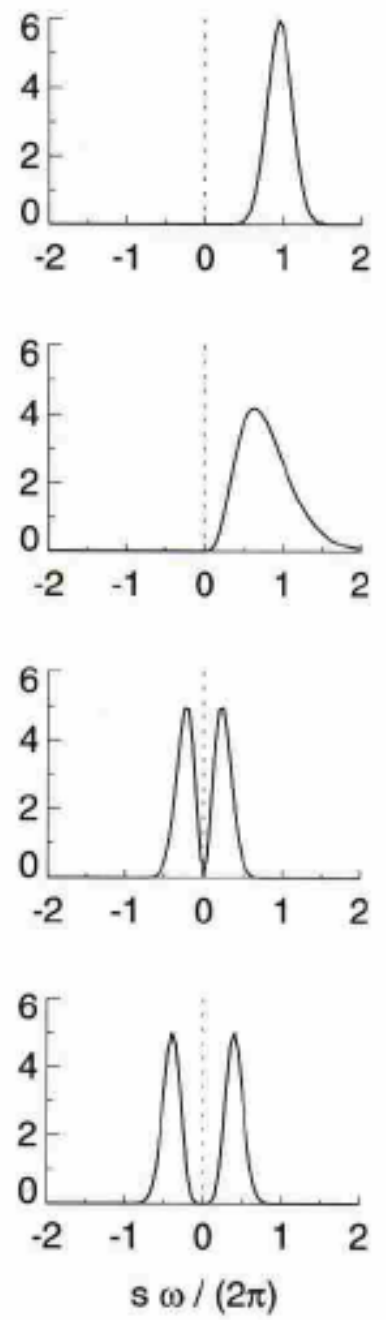

Figure 4.6. Four different wavelet functions. Left column is in the time domain with solid lines indicating the real part of the wavelet and the dashed lines indicating the imaginary portion. Right column is in the frequency domain. (a) is the complex Morlet wavelet chosen for the experiments presented here. (b) Paul $m=4$ (c) DOG $m=2$ also known as the Mexican Hat and (d) DOG $m=6$. DOG stands for derivative of a Gaussian. Figure obtained from Ref. [20]. 


\subsection{Time-Frequency Analysis Examples}

In a recent investigation of the well-documented evolution of ion heating in the edge of the WVU HELIX plasma source, ${ }^{32,33}$ new time resolved fluctuation measurements in pulsed plasmas were performed to investigate possible ion heating mechanisms. ${ }^{26}$ Fig 4.7 shows the result of a continuous wavelet transformation of a time series obtained from a single tip electrostatic probe placed at various radial locations in the plasma column. The principal frequency components of the fluctuations (essentially integrated over all wave numbers since the measurements are from a single probe tip) versus time at $r=4.5 \mathrm{~cm}$ are shown in Figure 4.7a. The continuous wavelet transform of the time series sampled at $50 \mathrm{MHz}$ employed a complex Morlet function with a large center frequency and broad bandwidth.

In Figure 4.7a, the driving wave dominates the frequency spectrum at $9.5 \mathrm{MHz}$. However, close inspection (Figure 4.7b) in the $100-300 \mathrm{kHz}$ range reveals multiple frequency peaks, the largest occurring at approximately $245 \mathrm{kHz}$. For purposes of comparison, the wavelet transform amplitude at $245 \mathrm{kHz}$ versus time is shown in Figure 4.7c for two radial locations. The continuous wavelet transform analysis provides, in contrast to a classic Fourier transform analysis, the time evolution of the frequency resolved fluctuation amplitude. The $245 \mathrm{kHz}$ fluctuation amplitude is substantially larger and increases in amplitude in a manner consistent with the temporal evolution of the simultaneously measured edge ion temperature. 


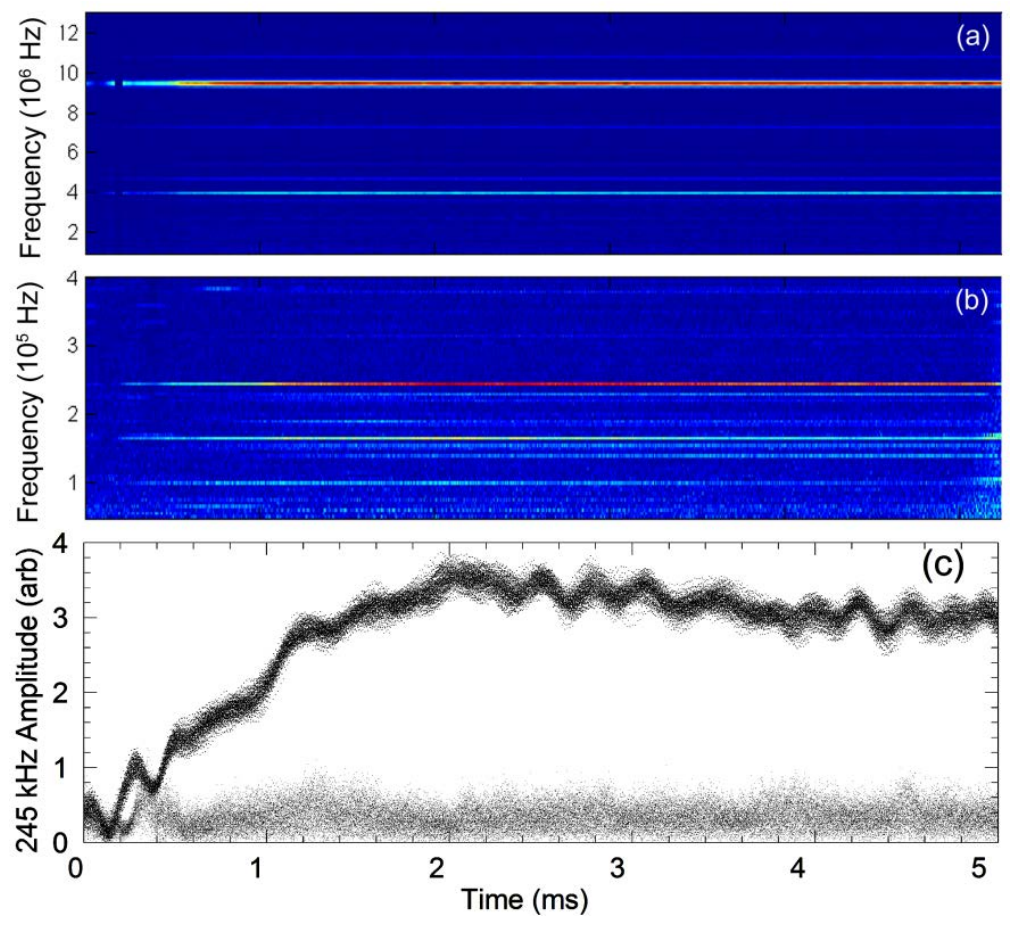

Figure 4.7. (a) Continuous wavelet transform of the first $5 \mathrm{~ms}$ of the electrostatic fluctuations measured at a radial location of $3.5 \mathrm{~cm}$. The narrow spectral feature at $9.5 \mathrm{MHz}$ is the primary rf wave for the source. (b) Expanded view of the lower frequency portion of the spectrum shown in (a). The amplitude of the peak at $~ 245 \mathrm{kHz}$ increases rapidly over the first2 ms of the discharge. (c) The amplitude of the wavelet power spectrum at $245 \mathrm{kHz}$ for radial locations of $0 \mathrm{~cm}$ (light gray) and $3.5 \mathrm{~cm}$ (black). Figure obtained from Ref. [26].

Although these measurements confirmed that the temporal behavior of the edge ion temperature is consistent with the growth of few hundred kilohertz, short wavelength waves in the plasma edge, they did not demonstrate the excitation of $\sim 10 \mathrm{MHz}$ waves by the source antenna. Evidence for the excitation of $10 \mathrm{MHz}$ waves appears in the details of the averaged fluctuation power spectrum measured during the pulsed discharge. Shown in Figure 4.8 is the averaged power spectrum (500 averages) based on a 5 ms wide acquisition window centered on $12.5 \mathrm{~ms}$ into the discharge at a radial location of $3.5 \mathrm{~cm}$. The primary driving frequency at 9.5 $\mathrm{MHz}$ is evident as is a higher frequency, electrostatic sideband $245 \mathrm{kHz}$ above the driving 
frequency of the discharge. The higher frequency wave satisfies the frequency matching condition for parametric excitation by the primary rf wave in the helicon discharge. Examination of power spectra obtained from additional $5 \mathrm{~ms}$ windows centered on $2.5 \mathrm{~ms}, 7.5 \mathrm{~ms}$, and 17.5 ms into the pulse indicates that the upper sideband amplitude tracks the temporal evolution of the $245 \mathrm{kHz}$ wave amplitude during the initial $20 \mathrm{~ms}$ of the plasma pulse. Because the continuous wavelet transform is based on a single time series measurement, noise is introduced and the frequency resolution is decreased because of the increased time resolution. A FFT based method using 100's of averages loses time resolution but gains frequency resolution.

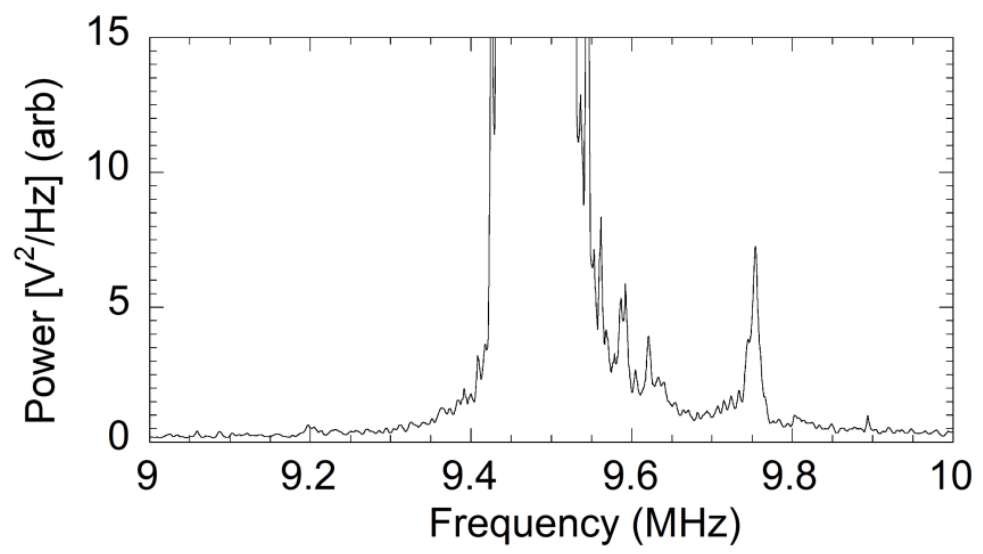

Figure 4.8. Spectral power versus frequency acquired during a $5 \mathrm{~ms}$ wide sampling window $12.5 \mathrm{~ms}$ into the $100 \mathrm{~ms}$ pulsed discharge. The peak of the driving frequency has been cut off to highlight the sideband wave at 9.745 MHz. Figure obtained from Ref. [26].

Thus, as shown with these Scime et al. experiments, both the wavelet and STFT analysis methods for obtaining time-resolved fluctuation information have particular strengths and together they provide a detailed picture of the evolution of the frequency components imbedded in a set of time series measurements. 


\section{Chapter 4 References}

${ }^{1}$ S. Chakraborty Thakur, Z. Harvey, I. A. Biloiu, A. Hansen, R. A. Hardin, W. S. Przybysz, and E. E. Scime, Phys. Rev. Lett. 102, 035004 (2009).

${ }^{2}$ K.M. Lau and H. Weng, Bull. Amer. Meteor. Soc., 76, 2391 (1995).

${ }^{3}$ E. E. Scime, I. A. Biloiu, J. Carr, Jr., S. Chakraborty Thakur, M. Galante, A. Hansen, S. Houshmandyar, A. M. Keesee, D. McCarren, S. Sears, C. Biloiu, and X. Sun, Phys. Plasmas 17, 055701 (2010).

${ }^{4}$ L. P. Block, Astrophys. And Space Sci. 55, 59 (1978).

${ }^{5}$ S. Iizuka, K. Saeki, N. Sata, and Y. Hatta, Phys. Rev. Lett. 43, 1404 (1979).

${ }^{6}$ C. Chan, N. Hershkowitz, and T. Intrator, Phys. Rev. Lett. 57, 3050 (1986).

${ }^{7}$ T. Sato and H. Okuda, Phys. Rev. Lett. 44, 740 (1980).

${ }^{8}$ M. J. Alport, S. L. Cartier, and R. L. Merlino, J. Geophys. Res. 91, 1599 (1986).

${ }^{9}$ A. Aanesland, C. Charles, M. A. Lieberman, and R. W. Boswell, Phys. Rev. Lett. 97, 075003 (2006).

${ }^{10}$ S. P. Gary and N. Omidi, J. Plasma Phys. 37, 45 (1987).

${ }^{11}$ I. A. Biloiu and E. E. Scime, Appl. Phys. Lett. 95, 051504 (2009).

${ }^{12}$ R.X. Gao and R. Yan, in Wavelets: Theory and Applications for Manufacturing, (Springer Science+Business Media, LLC, New York, 2011).

${ }^{13}$ C.K. Chui, in An Introduction to Wavelets, (Academic Press, San Diego, 1992).

${ }^{14}$ R. N. Bracwell, in The Fourier Transform and Its Applications, (McGraw-Hill Book Company 1978). 
${ }^{15}$ J.S. Walker, in A Primer on WAVELETS and Their Scientific Applications, (Chapman \& Hall/CRC Taylor \& Francis Group, LLC, Boca Raton, 2008).

${ }^{16}$ M. T. Heideman, D. H. Johnson, C. S. Burrus, IEEE ASSP Mag. (October 1984).

${ }^{17}$ A. V. Oppenheim and R.W. Schafer, in Discrete-Time Signal Processing, (Prentice-Hall, Upper Saddle River, 1999).

${ }^{18}$ P. Flandrin, in Time-Frequency/Time-Scale Analysis, (Academic Press, San Diego, 1999).

${ }^{19}$ B. B. Hubbard, in The World According to Wavelets: The Story of a Mathematical Technique in the Making, (A K Peters, Natick 1998).

${ }^{20 .}$ C. Torrence and G. P. Compo, Bull. Amer. Meteor. Soc., 79, 61 (1998).

${ }^{21}$ C. Torrence and P. J. Webster, J. Climate, 12, 2679 (1999).

${ }^{22}$ B. Ph. van Milligen, C. Hidalgo, and E. Sánchez, Phys. Rev. Lett., 74, 395 (1995).

${ }^{23}$ F. Brochard, T. Windisch, O. Grulke, and T. Klinger Phys. Plasmas, 13, 122305 (2006).

${ }^{24}$ T. Kobayashi, S. Inagaki, S.-I. Itoh, K. Ida, S. Oldenbürger, H. Tsuchiya, Y. Nagayama, K. Kawahata, H. Yamada, M. Sasaki, A. Fujisawa, K. Itoh1, and the LHD Experiment Group Plasma Phys. Control. Fusion 53, 095012 (2011).

${ }^{25}$ S. Oldenbürger, S. Inagaki, T. Kobayashi, H. Arakawa, N. Ohyama, K. Kawashima, Y. Tobimatsu, A. Fujisawa, K. Itoh, and S.-I. Itoh, Plasma Phys. Control. Fusion, 54, 055002 (2012).

${ }^{26}$ E. E. Scime, J. Carr Jr., M. Galante, R. M. Magee, and R. Hardin, Phys. Plasmas, 20, 032103 (2013).

${ }^{27}$ M. Farge, Annu. Rev. Fluid Mech., 24, 395 (1992).

${ }^{28}$ Y. Meyer, in Wavelets: Algorithms \& Applications, (Society for Industrial and Applied Mathematics, Philadelphia, 1993). 
${ }^{29}$ I. Daubechies, in Ten Lectures on Wavelets, (Society for Industrial and Applied Mathematics, Philadelphia, 1992).

${ }^{30}$ G. Strang and T. Nguyen, in Wavelets and Filter Banks, (Wellesley-Cambridge Press, Wellesley 1997).

${ }^{31}$ X. Shi, J. Boman, and M. G. Shats, Rev. Sci. Instrum., 72, 503 (2001).

${ }^{32}$ J. L. Kline, E. E. Scime, R. F. Boivin, A. M. Keesee, and X. Sun, Plasma Sources Sci. Technol. 11, 413 (2002).

${ }^{33}$ J.L. Kline, E.E. Scime, R.F. Boivin, A.M. Keesee, X. Sun, and V.S. Mikhailenko, Phys. Rev. Lett., 88, 195002 (2002). 


\section{Chapter 5: Experimental Results and Discussion}

\subsection{Time Resolved LIF Double Layer Studies}

Experimental results presented in Chapter 4 showed that in HELIX, large amplitude, low frequency fluctuations appear in the helicon source plasmas when the double layer structure vanishes. Other previous experiments demonstrated that formation of the DL in pulsed helicon source plasmas occurs a few ms after the initiation of the discharge (see Fig. 3.20 for an example of a time-resolved IVDF measurement of a beam and background ion population). To determine if there is a causal relationship between the DL and the observed ion acoustic waves, the complete IVDF and the plasma potential fluctuation amplitude were measured as a function of time throughout 100 ms discharges (5 Hz repetition rate, 50\% duty cycle) with and without a DL in steady-state plasmas at the same source parameters.

\subsubsection{Beam Formation and Collapse}

Shown in Fig. 5.1a is a measurement of the complete IVDF 5 ms into a pulsed discharge for an antenna frequency of 9.0 MHz, a source magnetic field of $1000 \mathrm{G}$, an expansion chamber magnetic field of $17 \mathrm{G}$, and a neutral pressure of 0.98 mTorr. ${ }^{1}$ For this set of parameters, the mirror ratio (upstream to downstream) is 60. The IVDF measurement includes 80 distinct laser frequencies and was averaged over 200 plasma pulses with a lock-in amplifier integration time of $1 \mathrm{~ms}$ (yielding an effective time resolution of a few milliseconds). Shown in Fig. 5.1b is the average of 200 measurements of the time-resolved floating potential fluctuation amplitude measured in the plasma source approximately $100 \mathrm{~cm}$ upstream of the DL. Note the appearance 
of a well-defined, coherent oscillation after the large peak in fluctuation amplitude at $21.7 \mathrm{~ms}$ into the pulsed discharge. The initial wave amplitude is quite large and the envelope of the fluctuations decays exponentially after the initial large peak. The fact that this fluctuation waveform survives averaging over 200 pulses demonstrates the highly reproducible nature of these pulsed helicon discharges.
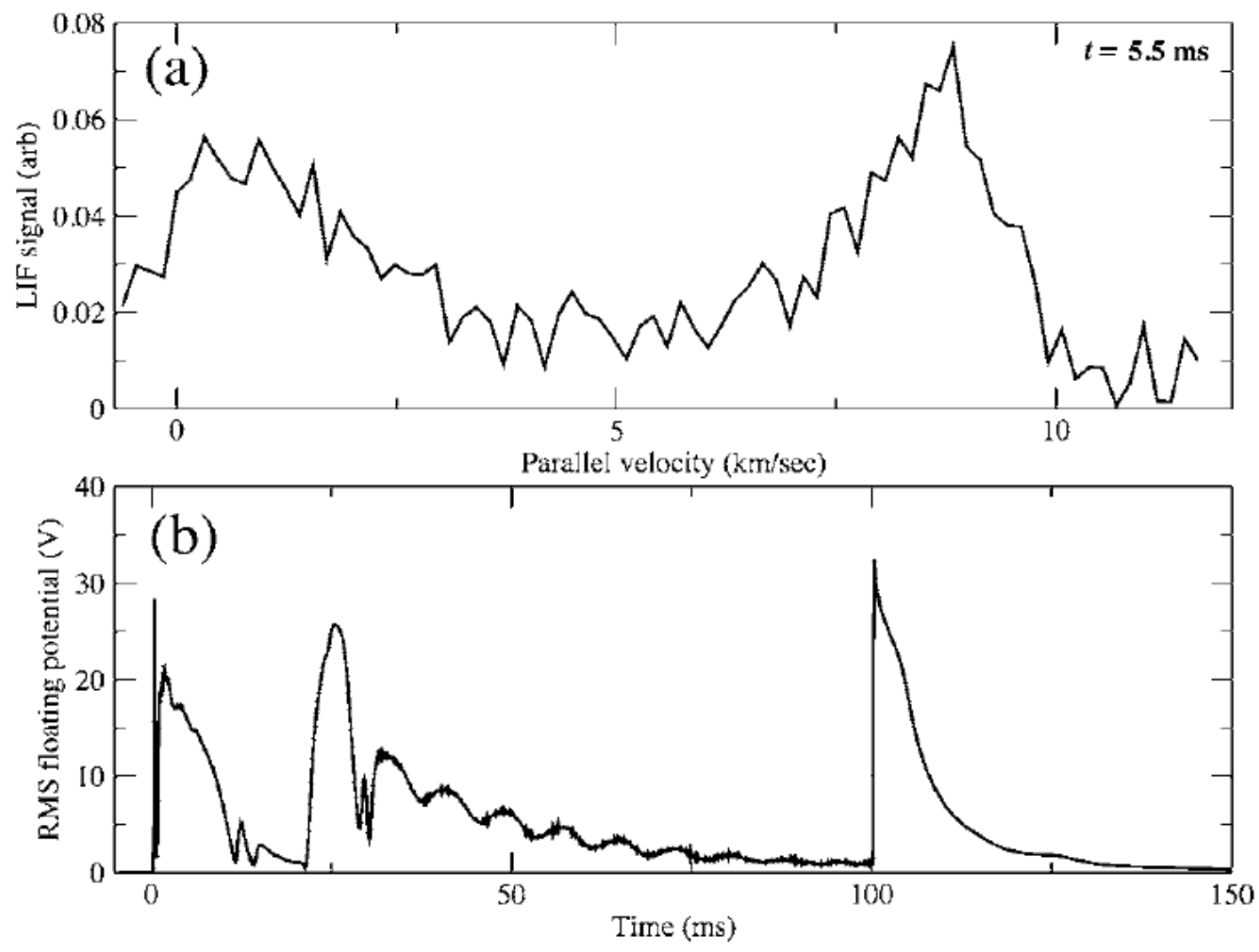

Figure 5.1. For an antenna frequency of $9.0 \mathrm{MHz}$, mirror ratio of 60. (a) The downstream IVDF $5.5 \mathrm{~ms}$ after the start of the $100 \mathrm{~ms}$ long pulsed discharge. Both the background population (left) and ion beam (right) are evident. (b) Time series of the fluctuating floating potential from a single tip of the multi-tip probe averaged over 200 pulses. The large spike in fluctuation amplitude begins at $21.7 \mathrm{~ms}$ into the discharge. The large spike at $t=100 \mathrm{~ms}$ is the termination of the pulse. Figure obtained from Ref. [1]. 
Just before the large spike in fluctuation amplitude, at $21.5 \mathrm{~ms}$, the ion beam completely vanishes (Fig. 5.2). For this large mirror ratio case, the initial ion beam velocity, when the beam first appears in the measured IVDF, was $8.7 \mathrm{~km} / \mathrm{s}$ and the background ion population to beam density ratio is roughly 1:1. The time difference between the collapse of the DL (termination of the ion beam) and the peak in fluctuation amplitude, $\sim 0.2 \mathrm{~ms}$, is consistent with the $0.14 \mathrm{~ms}$ required for a wave to propagate from the DL location to the fluctuation measurement location at the local sound speed.

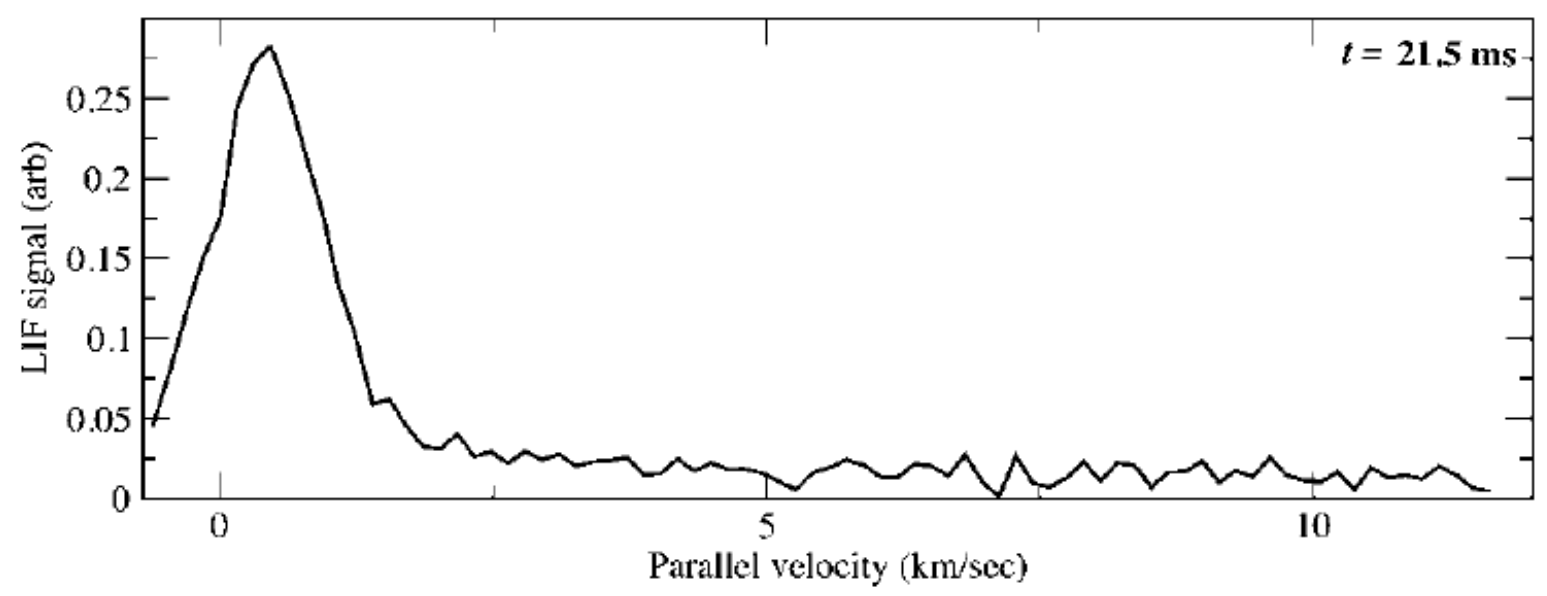

Figure 5.2. Same case as Fig. 5.1 but further in time, the downstream IVDF $21.5 \mathrm{~ms}$ ( $0.2 \mathrm{~ms}$ prior to the spike in fluctuation amplitude in Fig. 5.1b) after the start of the $100 \mathrm{~ms}$ long pulsed discharge. The background population is evident, but the ion beam has vanished. Figure obtained from Ref. [1].

For the same source magnetic field, the same neutral pressure, but for a larger expansion chamber magnetic field of $33 \mathrm{G}$ (which previous experiments demonstrated lead to reduced ion beam velocity, i.e., a weaker DL, because the mirror ratio of 30 is smaller than the previous 
case $^{2}$ ), the ion beam persists throughout the discharge. The IVDF $25.5 \mathrm{~ms}$ into the pulsed discharge is shown in Fig. 5.3a and a clear ion beam is still discernible. Note that for this case, the background ion population to ion beam density ratio is greater than $2: 1$ and the initial ion beam velocity was $7.3 \mathrm{~km} / \mathrm{s}$. The floating potential fluctuation amplitude versus time for these plasma conditions is shown in Fig. 5.3b. By $21.7 \mathrm{~ms}$ into the discharge, the time at which the large spike in fluctuation amplitude appeared in the previous case, the plasma potential fluctuation amplitude is nearly zero.
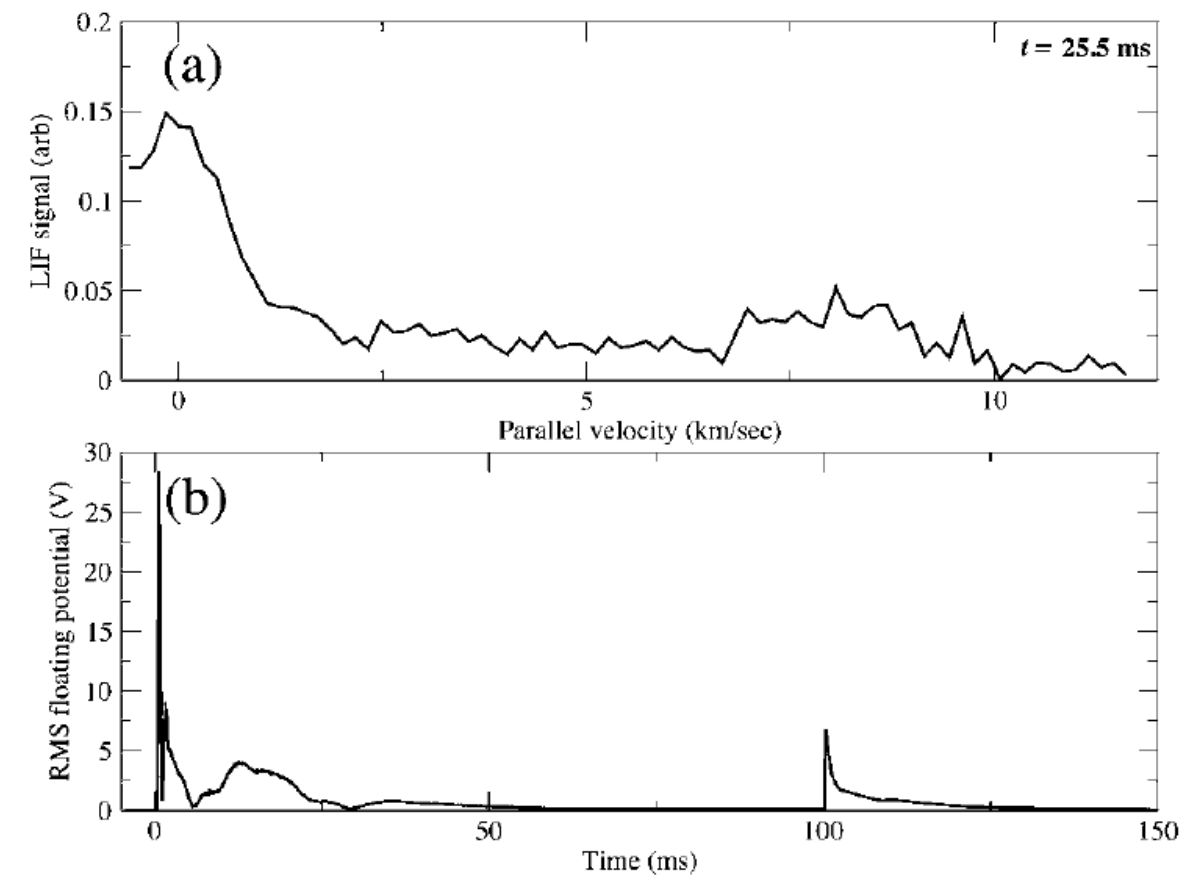

Figure 5.3. For an antenna frequency of $9.0 \mathrm{MHz}$ mirror ratio of 30. (a) The downstream IVDF $25.5 \mathrm{~ms}$ after the start of the $100 \mathrm{~ms}$ long pulsed discharge. Both the background population (left) and ion beam (right) are evident. (b) Time series of the fluctuating floating potential from a single tip of the multi-tip probe averaged over 200 pulses. Figure obtained from Ref. [1]. 
As shown in the composite image in Fig. 5.4a, for the larger mirror ratio, the initial beam population is actually larger than the background ion population at this measurement location. As the beam decays, the background ion population becomes much larger than the beam population. The floating potential fluctuation amplitude versus time is shown beneath the time-resolved IVDF in Fig. 5.4a and there is a clear anti-correlation between the fluctuation amplitude and the beam amplitude. When the fluctuation amplitude peaks, the beam amplitude is a minimum; leading to a "ripple" in both the IVDF and wave amplitude versus time. Shown in Fig. 5.4b are the same measurements for the smaller mirror ratio case. For the smaller mirror ratio, the relatively less dense (compared with the background ion population) and slower ion beam persists throughout the pulse and no instability is excited. The potential measurements shown in Fig. 5.4b are plotted on the same scale as used in Fig. 5.4a and it is clear that the fluctuation amplitude is much smaller for the smaller mirror ratio case. 

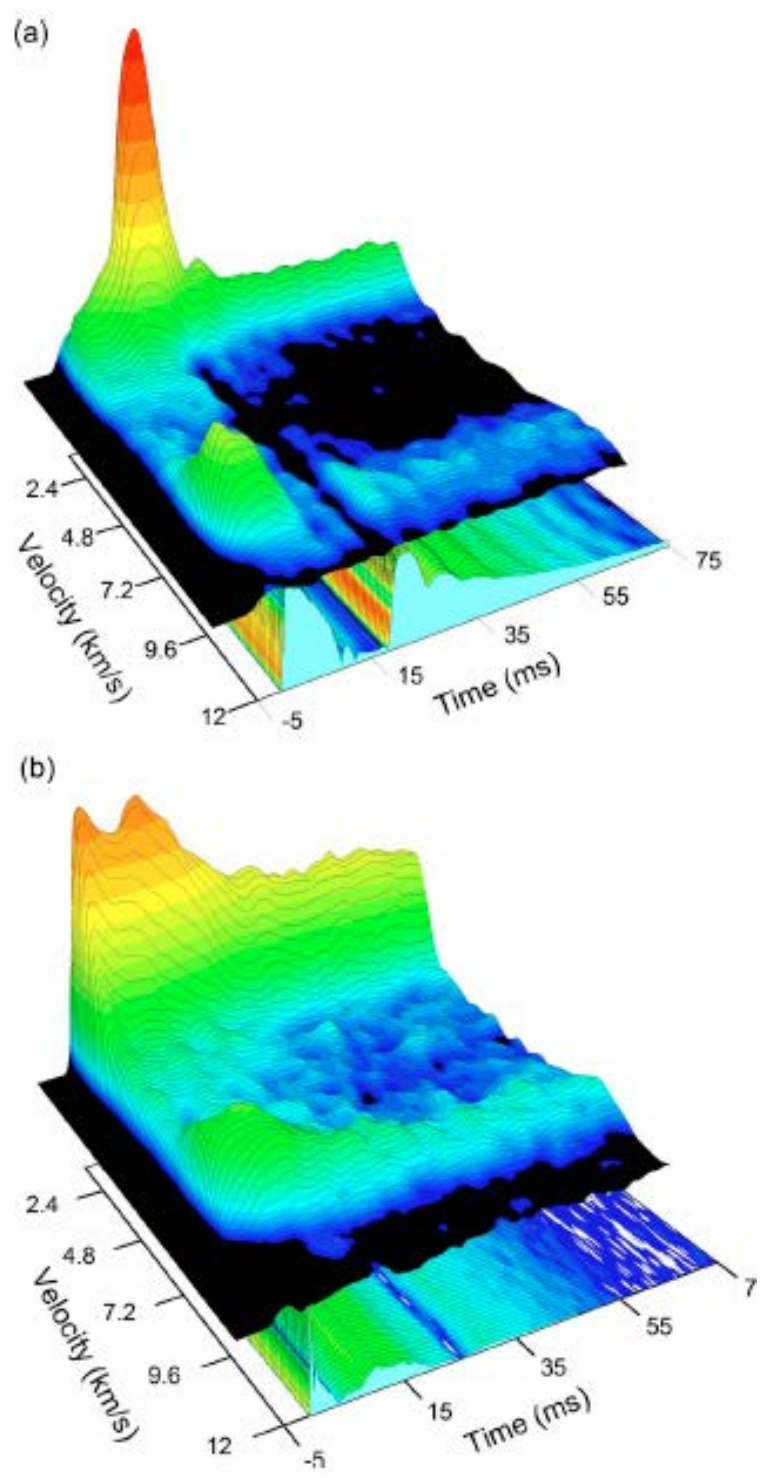

Figure 5.4 (a) For a mirror ratio of 60 (corresponding to Figs. 5.1 and 5.2), the upper surface plot is the IVDF versus time measured downstream of the DL. The ion beam appears at the start of the plasma pulse at a velocity of $\sim 8.7$ $\mathrm{km} / \mathrm{s}$ and is larger than the background population. The lower surface plot is the floating potential versus time measured upstream of the DL. (b) The same measurements as in part (a) for a mirror ratio of 30 (corresponding to Fig. 5.3). Figure obtained from Ref. [1]. 


\subsubsection{Analysis of Time Resolved Double Layer Study}

Low pressure, CFDL operational plasma parameters similar to those used in Chakraborty Thakur et al. ${ }^{12}$ were obtained and investigated with time resolved LIF studies. For the $9 \mathrm{MHz}$ driving frequency case, a DL once again did not appear during CW operation but large electrostatic instabilities were detected, consistent with Chakraborty Thakur et al. The time difference between the collapse of the DL (termination of the ion beam) and the peak in fluctuation amplitude, $\sim 0.2 \mathrm{~ms}$, was consistent with the $0.14 \mathrm{~ms}$ required for a wave to propagate from the DL location to the fluctuation measurement location at the local sound speed. Time resolved measurements in these pulsed discharges indicate that the double layer initially forms for all tested mirror ratios and persists throughout the pulse duration for the moderate mirror ratio case of 30. These data further support the hypothesis that for particularly strong double layers, the instability appears early in the discharge and the double layer collapses.

These observations of beam collapse motivated additional, higher time resolution experiments. Establishment of a causal link between DLs and the instability requires that the beam population, and not the background, show evidence of statistically significant correlation with the instability. With an instability wave of $17.5 \mathrm{kHz}$ and a sub $1 \mathrm{kHz}$ bandwidth limit on LIF measurements imposed by the lock-in amplifier, higher time resolution LIF studies were required. 


\subsection{High Time Resolution Studies}

Downstream of the expansion region, a clear ion beam (evidence of a double layer) is seen only for higher magnetic mirror ratios. To investigate the ion beam formation with improved time resolution, LIF measurements were obtained using the smallest possible time constant on the lock-in amplifier. The plasma was pulsed at $5 \mathrm{~Hz}$ with a 50\% duty cycle. Fig. 5.5 shows the three dimensional time resolved LIF scans obtained for two different magnetic field mirror ratios, one that resulted in a DL and one that did not. The same data are shown in Fig. 5.6 compressed into a two-dimensional plot for added clarity. Fig 5.5a is for a downstream magnetic field of 34 Gauss while Fig 5.5b is for a downstream field of 17 Gauss. The source parameters shared by both cases are: a driving frequency of $9 \mathrm{MHz}$, an upstream magnetic field of 700 Gauss, $750 \mathrm{~W}$ rf power measured at the output of the rf amplifier, and a downstream pressure of $10^{-5}$ Torr. There was a slight difference in the upstream operating pressure (1.8 mTorr versus 1.5 mTorr for Figures 5.5a and 5.5b, respectively), that arose from the difference in the downstream magnetic field. Both pressures ware still within ranges typical of those for which DLs have been observed in previous HELIX-LEIA experiments. The DL only appears in the larger mirror ratio case of Fig 5.5b. 
(a)

(b)
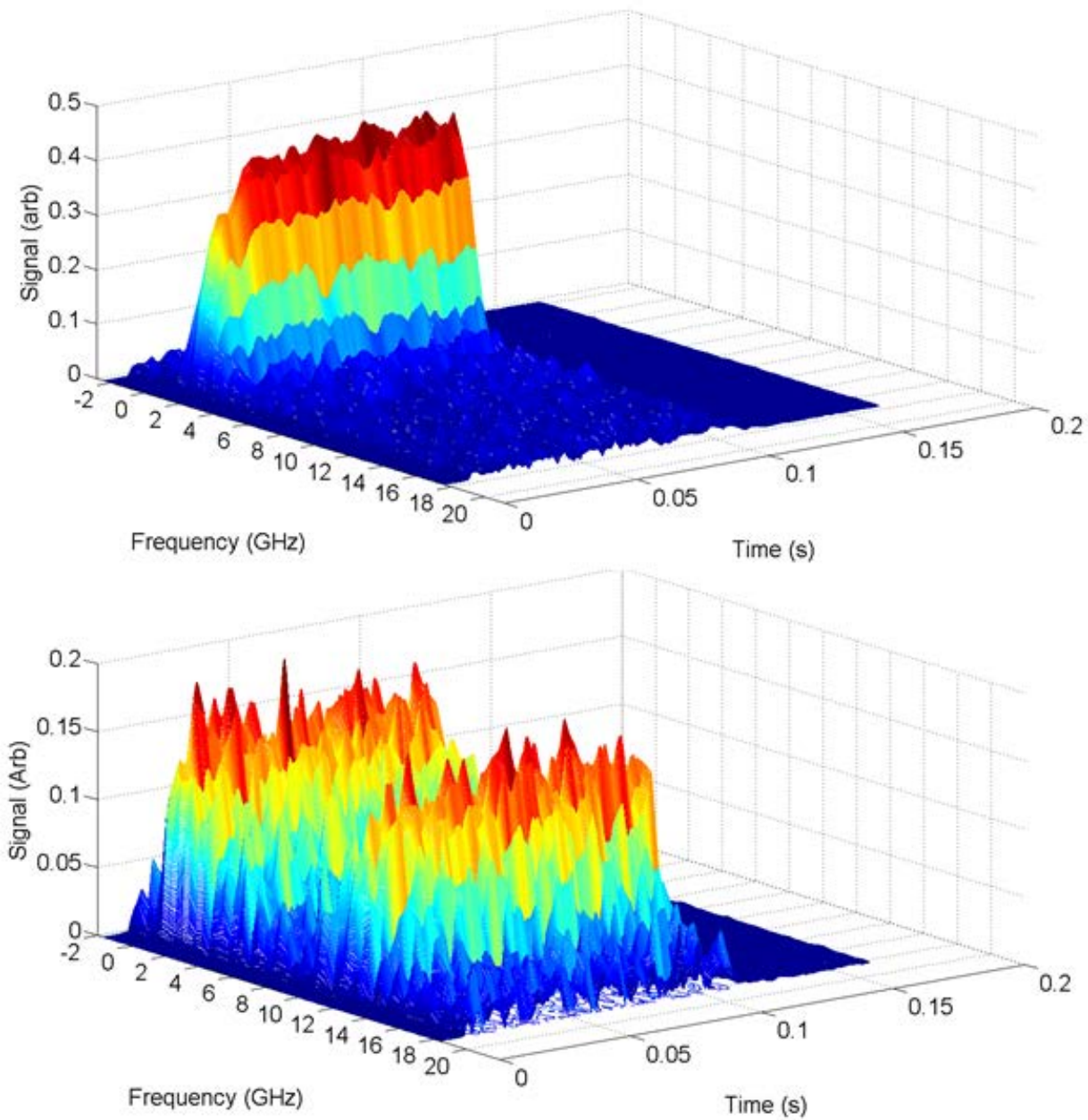

Figure 5.5. (a) The measured ion velocity distribution as a function of time and velocity (laser frequency) for an antenna frequency of $9.0 \mathrm{MHz}$ and a magnetic field of 34 Gauss in the expansion chamber; a mirror ratio of 22. For the downstream IVDF. Only the background population is evident after 200 averages. (b) The measured ion velocity distribution as a function of time and velocity (laser frequency) for an antenna frequency of $9.0 \mathrm{MHz}$ and a magnetic field of 17 Gauss in the expansion chamber; a mirror ratio of 44. For the downstream IVDF, both the bulk and beam populations are evident after 200 averages. The beam at times eclipses the bulk in signal intensity, but remains comparable in magnitude throughout most of the discharge. 
(a)

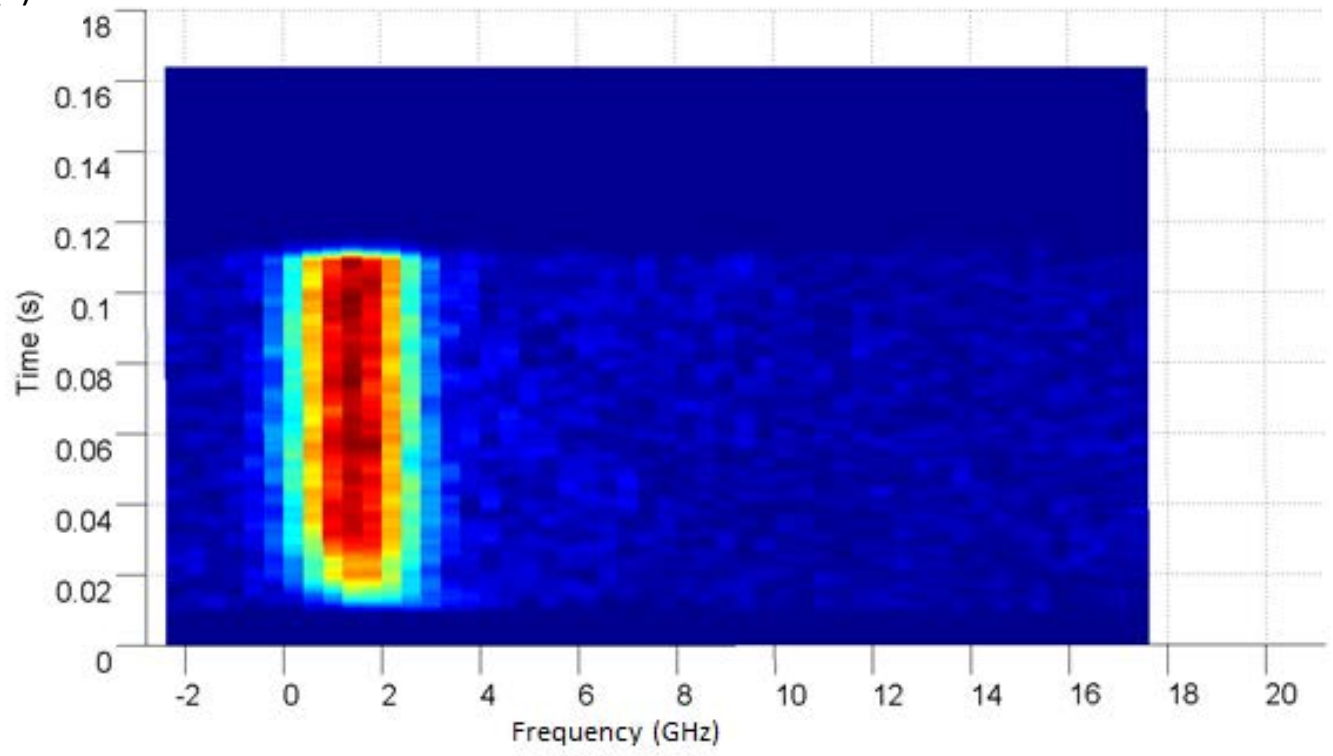

(b)

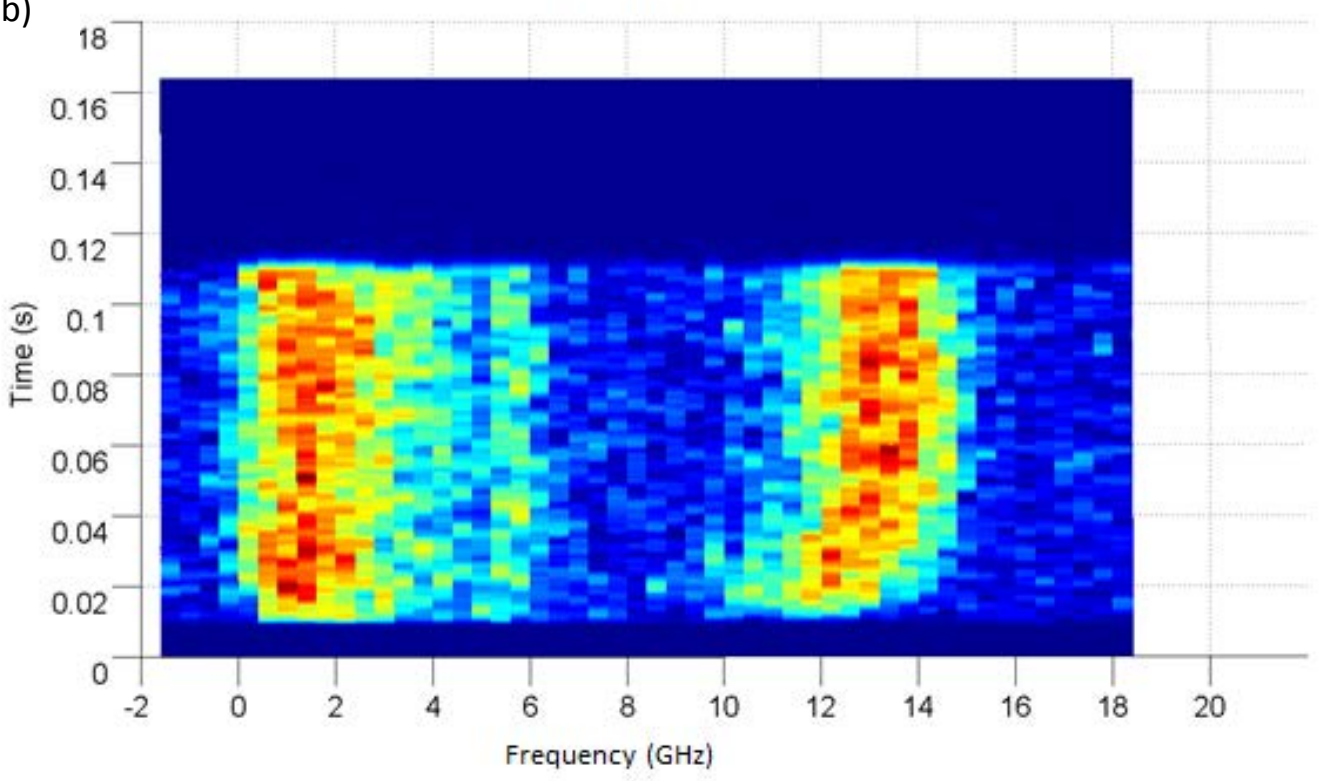

Figure 5.6. (a) Same data as shown in Fig 5.5a, but in two dimensions for clarity. (b) Same data as shown 5.5b but in two dimensions for clarity. The beam population velocity clearly increases in the initial $40 \mathrm{~ms}$ seconds, then maintains a steady velocity throughout the rest of the pulse. Velocity values for the bulk and beam populations using the non-lock-in LIF technique were chosen based on these data.

The IVDF in Fig. 5.5a contains a single slow population moving at $300 \mathrm{~m} / \mathrm{s}$, the background plasma flowing into the LEIA chamber at the sound speed. By comparison, Fig. 5.5b 
shows two populations, the background and the beam. The background plasma has a speed of $300 \mathrm{~m} / \mathrm{s}$ throughout the pulse while the beam starts at $6.5 \mathrm{~km} / \mathrm{s}$ and gradually increases up to 8 $\mathrm{km} / \mathrm{s}$. Note that there is a hint of a second, lower energy, ion beam population in Fig. 5.5b. Although the LIF signal clearly contains significant short timescale fluctuations, the $1 \mathrm{~ms}$ time constant on the lock-in amplifier still limited fluctuation analysis of LIF data to frequencies much less than $1 \mathrm{kHz}$.

\subsubsection{Beam - Fluctuation Cross Correlation}

Obtained for the larger magnetic field mirror (44) ratio case, Fig. 5.7 is a measurement of the cross power spectrum calculated for an entire $100 \mathrm{~ms}$ long time series measurement of the floating potential from the two tips of the electrostatic probe located in the source at location $\mathrm{C}$ in Fig 2.2 and sampled at $500 \mathrm{kHz}$. Fig 5.7 does not have any wave activity across the two tips. However a nonstationary wave that appears at the beginning of the pulse and nowhere else would be completely obscured by this measurement. 


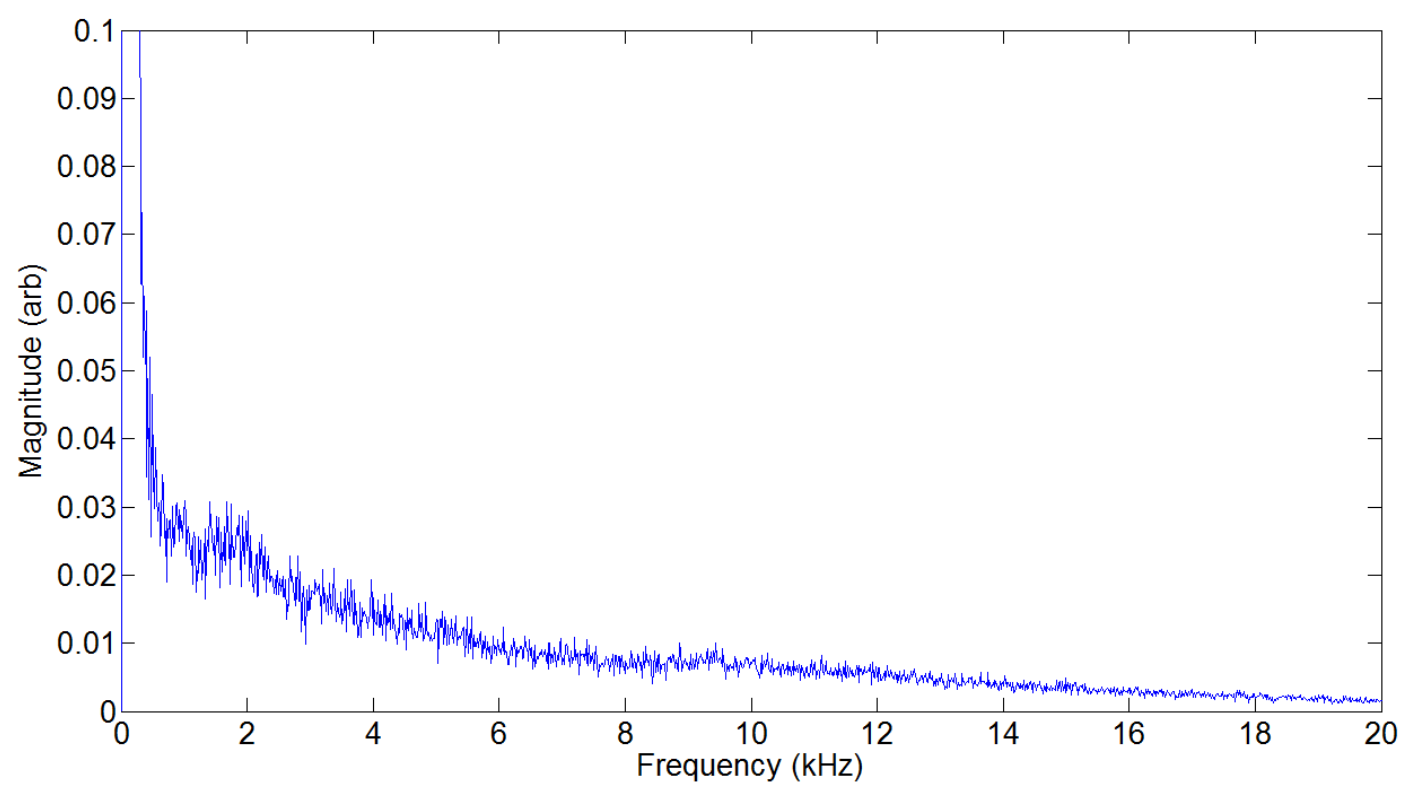

Figure 5.7. One hundred averages of a cross power spectrum from the 2 tips of the electrostatic probe. The maximum value of the vertical axis has been truncated to emphasize the peak at $\sim 2 \mathrm{kHz}$ and the lack of a peak in the tens of kilohertz band and beyond. Cross power spectrum peaks in amplitude for the lower frequencies occur at 50 (max) and $100 \mathrm{~Hz}$.

Evident in Fig 5.8 is a large amplitude fluctuation at approximately $17 \mathrm{kHz}$. Note that this is the same experimental configuration that results in the DL accelerated ion beam. Since the power spectrum measurement encompasses the entire plasma pulse, there is no way to determine if the wave exists throughout the entire discharge or if it only appears at certain times in the discharge. The time-frequency analysis is required to determine a true causal relationship between these waves and the beam. 


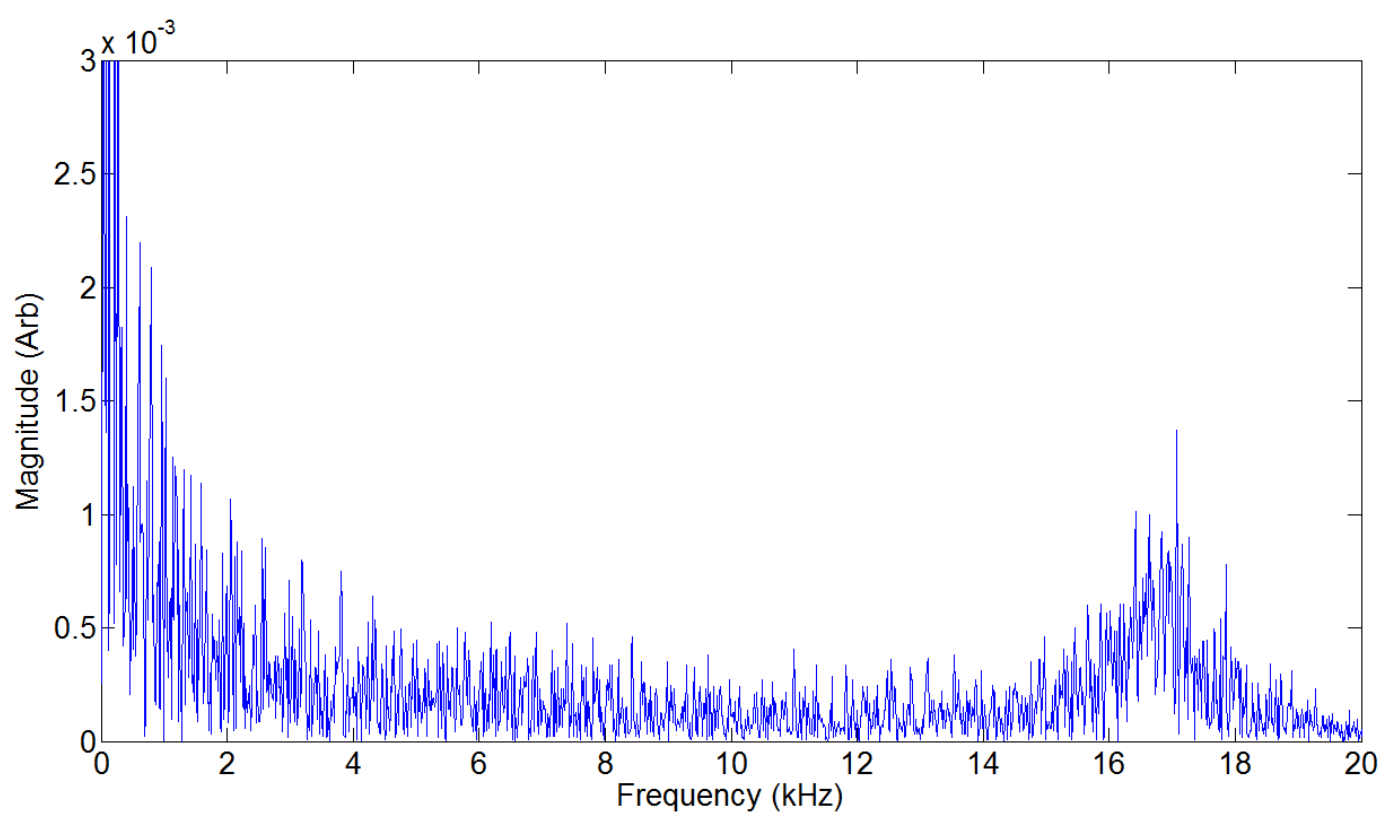

Figure 5.8. Five hundred averages of the FFT magnitude of a single tip of the electrostatic probe. The maximum value of the Y axis in this plot has been truncated to highlight the peak at $17 \mathrm{kHz}$. The FFT magnitude peaks at the lower frequencies occur at $180(\max )$ and $90 \mathrm{~Hz}$.

5.85.8To obtain true cross power spectrum measurements between the LIF and electrostatic signals, the Lock-in/AOM system was removed to maximize the frequency bandwidth of the LIF signal. As outlined in Section 3.5 and Chapter 4, the laser was set to specific frequencies which corresponded to the peak of the background and the peak of the beam signal as identified in the measurements shown in Fig. 5.6. The raw PMT signal and the electrostatic probe signal were then acquired with the same oscilloscope and processed with identical algorithms. After dividing the full time record into smaller intervals, the frequencyresolved cross power spectrum between specific ion velocity components (laser frequencies) and the probe fluctuations was determined as a function of time throughout the discharge. Shown in Figure 5.9 is the time resolved cross power spectrum between the LIF signal at the velocity of 
the peak of the ion beam and the electrostatic probe for the operating conditions used to obtain the beam observed in the IVDF shown in Fig 5.6b. Shown in Figure 5.10 is the time resolved cross power spectrum between the bulk of the ion distribution and the electrostatic probe for the exact same plasma conditions.

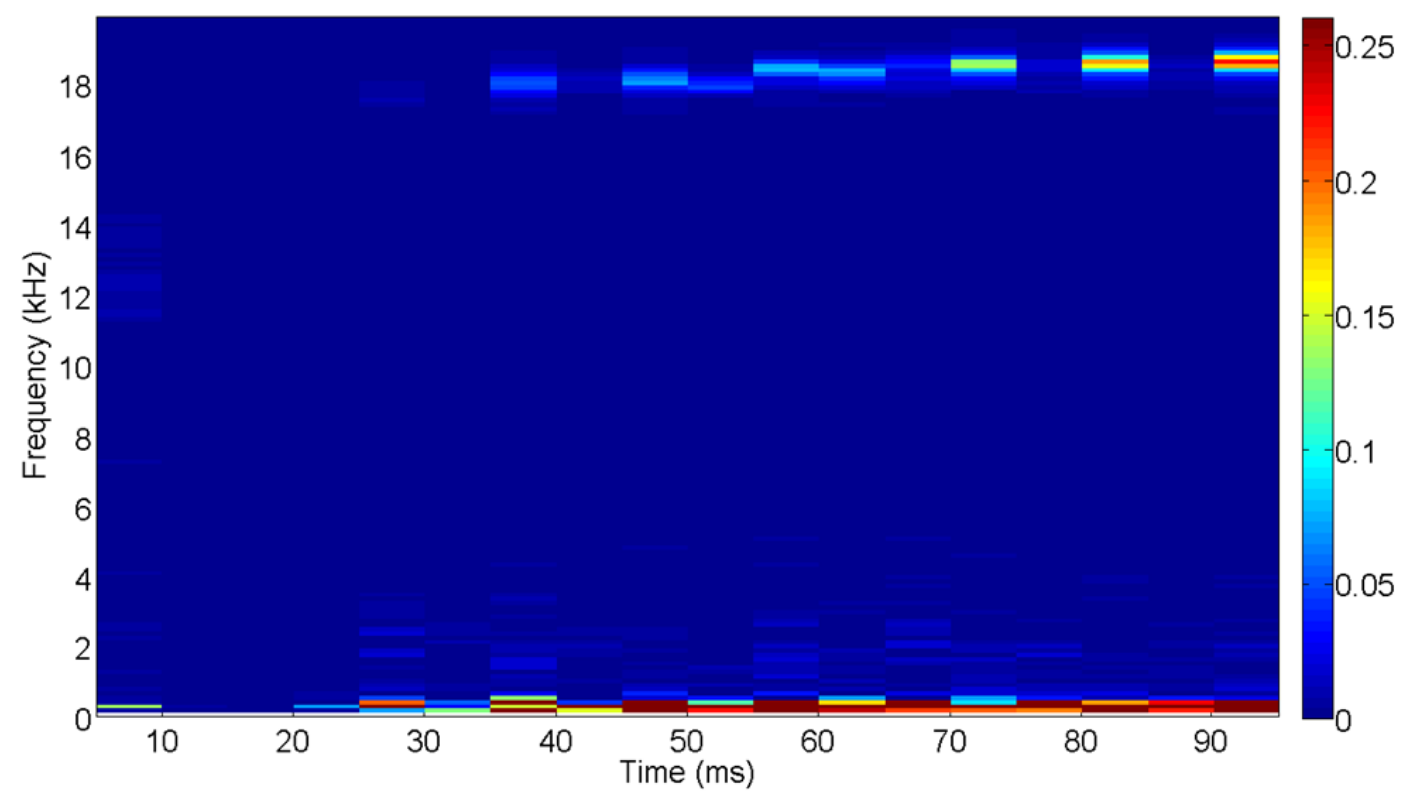

Figure 5.9. Five hundred averages cross power spectrum between the magnitude of the LIF signal at the peak of the beam in the ion velocity distribution and a single tip of the electrostatic probe. 


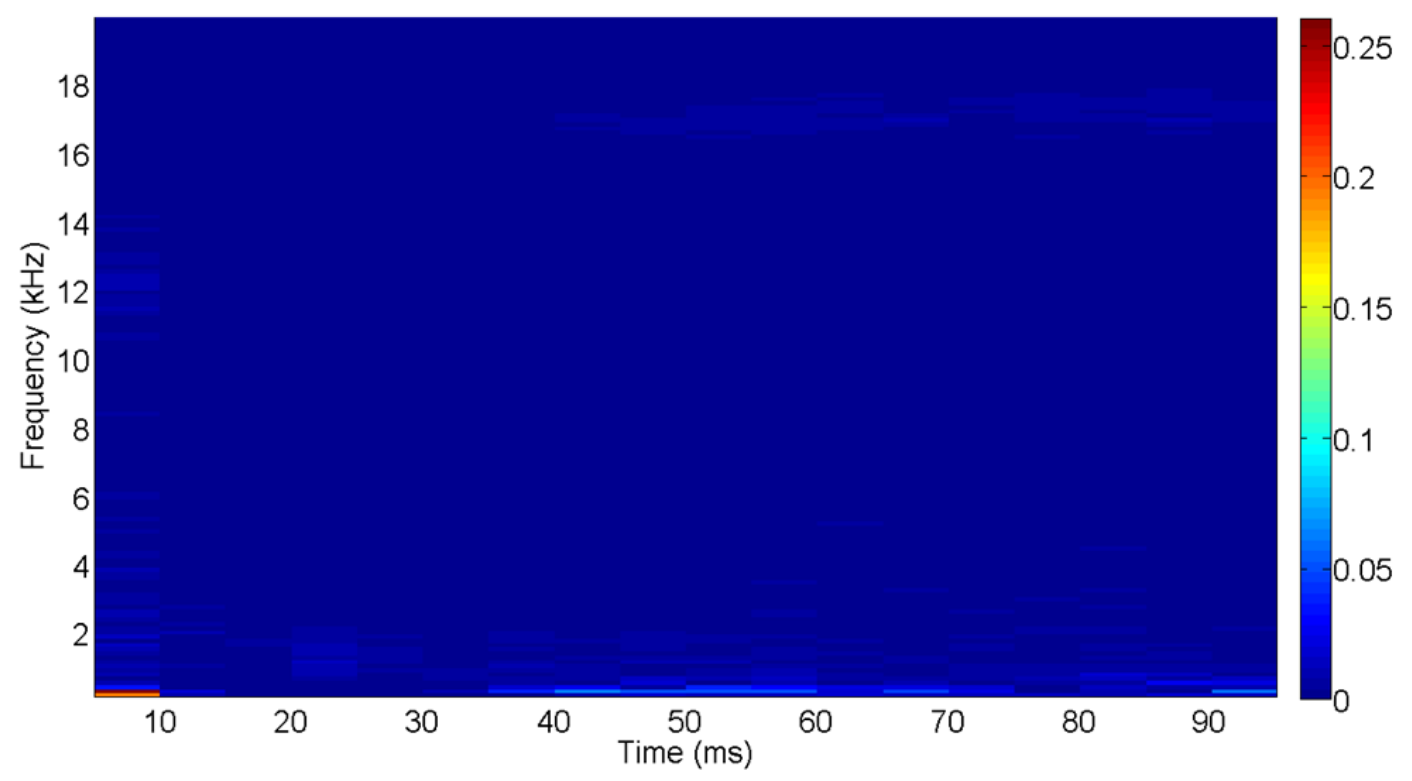

Figure 5.10. Five hundred averages of the cross power spectrum between the magnitude of the LIF signal at the peak of the bulk of the ion velocity distribution and a single tip of the electrostatic probe. The maximum value of the color map is not scaled to the peak of the very large correlation that appears at low frequency so as to emphasize the cross power magnitudes at $2 \mathrm{kHz}$ and $17 \mathrm{kHz}$.

For the beam-fluctuation comparison, there is a clear peak near $17 \mathrm{kHz}$ in the cross power spectrum. The $17 \mathrm{kHz}$ feature does not appear until approximately 40 ms into the pulse - the same time at which the beam velocity stabilizes in the data shown in Fig. 5.6. Throughout the rest of the pulse, the amplitude of the $17 \mathrm{kHz}$ feature in the cross power spectrum intensifies and fades with some regularity. The centroid frequency of the feature also increases up to approximately $18 \mathrm{kHz}$ by the end of the plasma pulse. Stronger correlations exist at lower frequencies, approximately $300-400 \mathrm{~Hz}$, and the magnitude of the cross correlation at the lower frequencies also increases and decreases throughout the pulse. The cross correlation between the bulk of the ion population and the electrostatic signal (shown in Fig. 5.10) is dramatically 
different. No significant features at any frequencies are observed. Note that the discontinuities in the intensity versus time are due to the discrete nature of the analysis.

These results are consistent with previous observations that suggest an ion acoustic or beam driven instability plays an important role in beam formation in expanding plasmas. Even though the time resolved cross power spectra are un-normalized, this study is the first to prove a correlation between DL and the ion acoustic instability.

\subsubsection{Wavelet-Based Fluctuation Analysis}

The cross-correlation analysis shown in Fig. 5.8 clearly identifies a correlation between the electrostatic fluctuation signal at $17 \mathrm{kHz}$ and the LIF signal from the beam population. However, the discrete nature of the method of analysis, FFTs over short time intervals, is somewhat unsatisfying in terms of demonstrating conclusively that the amplitude of the wave signal is modulated in time. Therefore, a continuous wavelet transform was applied to the time series data from the electrostatic probe to avoid discontinuities in the measurements. Fig 5.11 shows the results of the wavelet analysis of the electrostatic probe data. The time axis is continuous in the wavelet analysis, at the cost of a modest loss of frequency resolution. The nonstationary wave behavior is clearly captured in time by the wavelet analysis. Both the discrete FFT analysis and the wavelet analysis are consistent with the claim made in Section 5.1.2; as the wave activity oscillates, so does the beam amplitude. The continuous wavelet transform analysis indicates that the wave grows to some threshold amplitude then collapses and the process repeats. The period of this behavior is $\sim 10 \mathrm{~ms}$. These measurements also agree with Singh's prediction in his 2011 review article; the nature of the ion-acoustic mode leads to oscillatory behavior of turbulence and relaxation, causing the DL to appear, disappear and then reappear. ${ }^{3}$ 

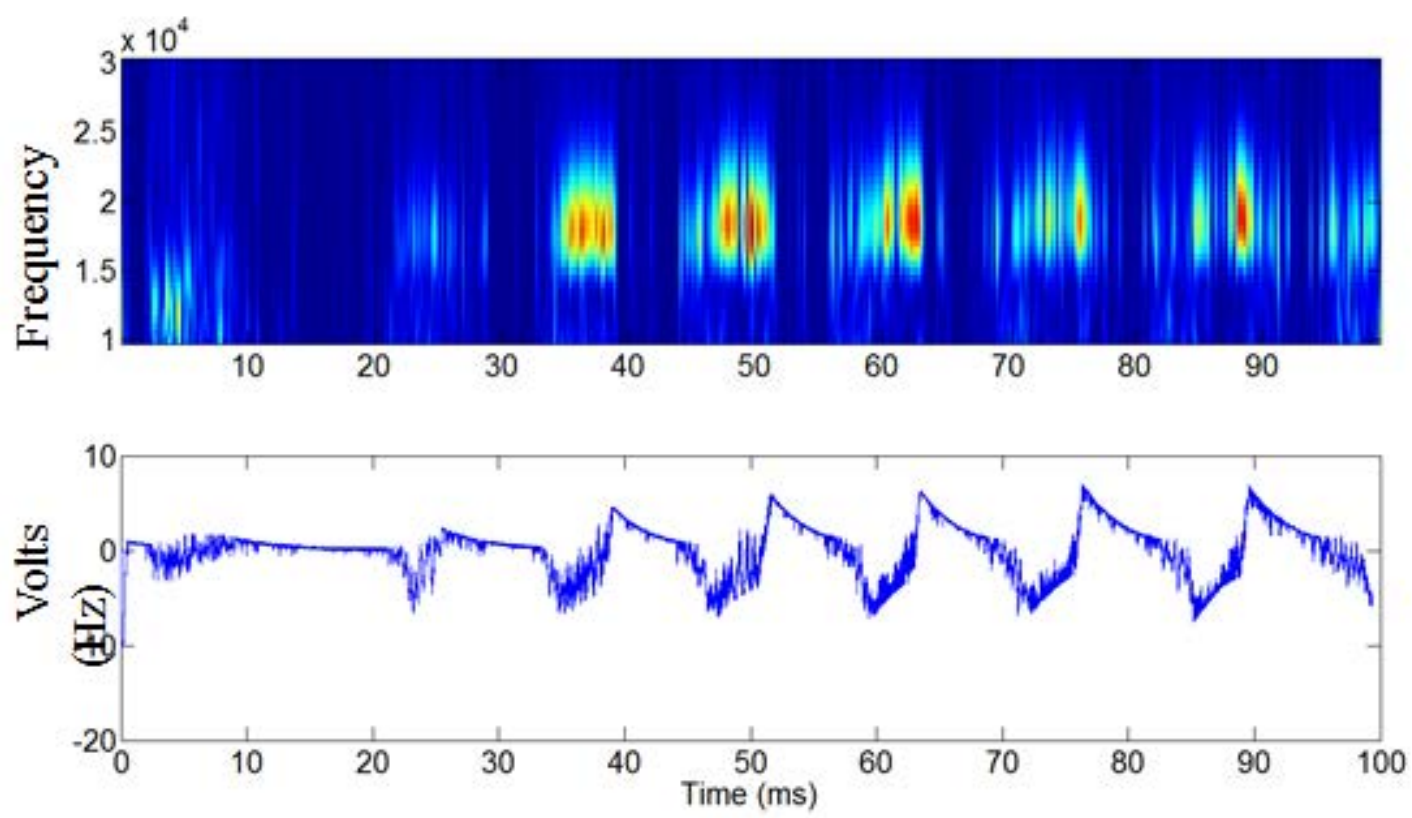

Figure 5.12. The continuous wavelet transform (top) of a single time series measurement (bottom) for the same experiment conditions as used for Fig. 5.6b.

\subsection{Observations of Multiple Ion Beams ${ }^{4}$}

Fig 5.6b has another interesting feature that almost went unnoticed. At $5.5 \mathrm{GHz}$, there is a smaller population of ions moving faster than the bulk but slower than the main beam. Certain plasma operating parameters would occasionally deliver a third population. Over the years, the WVU group became more proficient in tuning to source parameters that would show the presence of multiple beams. However, without a complete theoretical understanding of the origin of the extra ion beams, the phenomenon remained more a curiosity than a focus of intense study. 
When recent space observations in the Earth's plasma sheet provided evidence of complex IVDF structures that look nearly identical to the laboratory observations, a detailed study of the multiple beam phenomenon was initiated. This section describes multiple ion beam observations in three different realms of plasma physics and considers the possible implications of multiple beam formation in divergent magnetic fields.

\subsubsection{Laboratory Observations}

Shown in Fig. 5.12(a) is an LIF measurement of the IVDF in the expansion region, $38 \mathrm{~cm}$ downstream of the plasma source and expansion chamber junction. Three ion populations are evident in the measurement: a low speed "bulk" population and two ion "beam" populations. The source parameters are a driving frequency of $9 \mathrm{MHz}$, an operating pressure of $1.8 \mathrm{mTorr}$, an upstream magnetic field of 700 Gauss, and a downstream magnetic field of 19 Gauss. The ion gyroradius in the source is approximately $0.5 \mathrm{~cm}$ and $13 \mathrm{~cm}$ in the expansion chamber. Operating pressures of 1.8 mTorr are atypically low for this experimental facility and stable, steady-state, operation of the plasma source required large levels of total rf power (800 $\mathrm{W}$, measured at the output of the rf amplifier) and careful minimization of the reflected rf power (less than $50 \mathrm{~W}$, measured at the input to the matching network). 

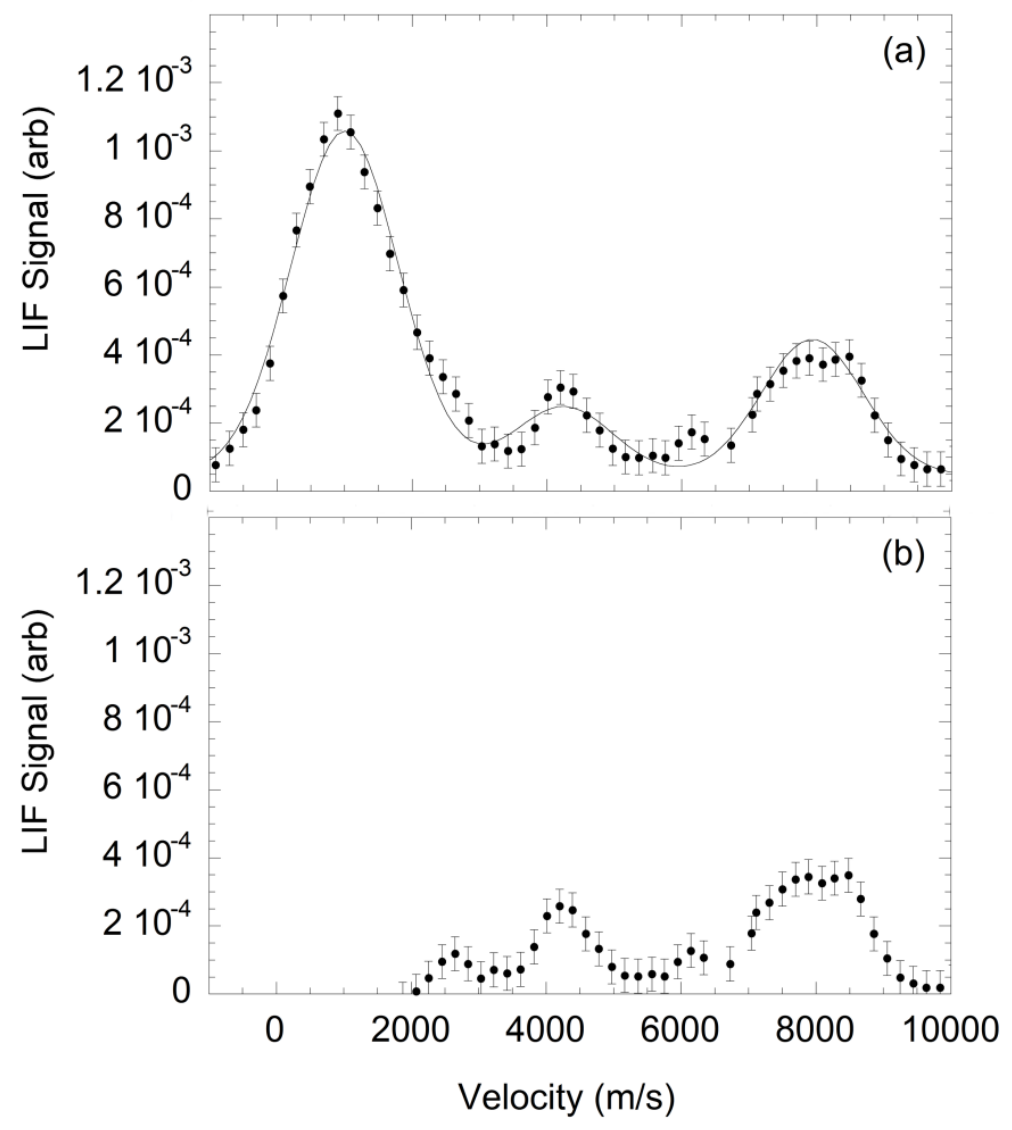

Figure 5.12. (a) LIF measured IVDF (circles) as a function of velocity in the expansion chamber $38 \mathrm{~cm}$ downstream of the plasma source. A three Maxwellian component fit (solid line) yields identical ion temperatures of $\sim 0.16 \mathrm{eV}$ for all three components. (b) Same data as (a) minus the fit to the stationary background population. A very small third accelerated population appears around 2,500 m/s.

The LIF-measured ion population upstream of the expansion region is well described by a single Maxwellian velocity distribution with a bulk ion temperature of $0.2 \mathrm{eV}$ and a bulk flow towards the expansion region of $\sim 300 \mathrm{~m} / \mathrm{s}$. Previous studies demonstrated that a DL forms at the junction between the source and the expansion chamber. The DL is localized in the region of the strongest magnetic field gradient where the density gradient is also largest. ${ }^{5}$ The highest energy 
population evident in Figure 5.12a, with a flow speed of $\sim 8,000 \mathrm{~m} / \mathrm{s}$, corresponds to an argon beam energy of $\sim 13 \mathrm{eV}$. Based on Langmuir probe measurements, the electron temperature in the source is $\sim 6 \mathrm{eV}$. Since the DL potential energy is roughly twice the electron temperature, these DLs are what are commonly called "weak" DLs. The second accelerated ion population that appears around $\sim 4,200 \mathrm{~m} / \mathrm{s}$ corresponds to an accelerating potential of $\sim 4 \mathrm{~V}$. An enhanced flux of ions in a narrow energy band is consistent with observations of ion beams in space plasmas typically attributed to $\mathrm{DLs}^{6}$ (whereas fluxes over a broad band of energy are typically attributed to stochastic acceleration in the electric fields arising from turbulent wave activity ${ }^{7}$ ).

Additional LIF measurements along the axis of the experiment show that while the intensity of the LIF signal decreases with distance from the acceleration region (consistent with quenching of the metastable argon ion state by electron-ion collisions as seen in previous experiments $^{8,9}$ ), the velocities of accelerated ion peaks in IVDF measurements are unchanged with distance (over 10's of $\mathrm{cm}$ ) from the acceleration region (see Figure 5.13). Although collisions with background electrons do depopulate the initial metastable state necessary for the LIF measurement, the momentum loss to the electrons and any background neutrals is negligible over the distances of these measurements. The persistence of the beam energies downstream of the DL is also independently confirmed with retarding field energy analyzer (RFEA) ${ }^{10}$ measurements. These axially resolved LIF measurements confirm that the ion beams are created upstream of the measurement location and can travel for tens of centimeters without significant degradation in beam energy. 


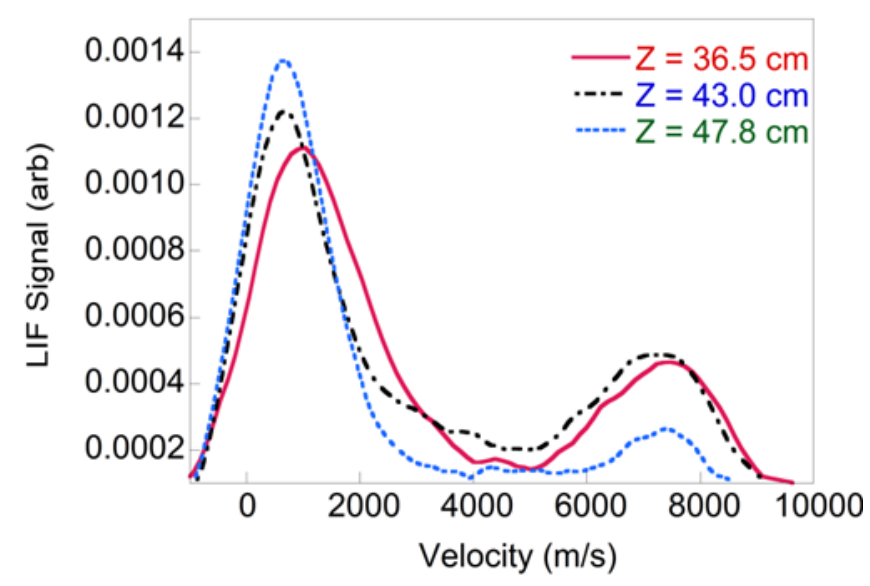

Figure 5.13. Smoothed fits to LIF measured IVDFs for a single double layer case as a function of velocity in the expansion chamber at three different downstream locations. Over more than $11 \mathrm{~cm}$, there is no change in the velocity of the accelerated ion population.

To understand how it is possible for ion beams at different energies to appear at a single downstream measurement location, we note that this is not the first time multiple accelerated ion populations have been observed in a helicon plasma. In a series of experiments on the Magnetized Nozzle eXperiment, multiple double layers were intentionally created by introducing grounded apertures upstream from a magnetic nozzle. ${ }^{11}$ Axially resolved LIF measurements demonstrated that ions accelerated in the sheath formed by the aperture traveled nearly $30 \mathrm{~cm}$ to the magnetic nozzle where they were further accelerated by the double layer at the magnetic nozzle. Between the grounded aperture and the magnetic nozzle, a single accelerated ion population and the background ions created beyond the aperture were both observed with LIF. Downstream of the second double layer, three distinct ion populations were evident: doubly accelerated ions, an accelerated ion population comprised of the ions created between the aperture and the magnetic nozzle that were then accelerated by only the double layer at the nozzle, and background ions. Thus, these earlier experiments demonstrated that multiple ion 
beams will appear in IVDFs when additional ionization occurs between accelerating structures, i.e., new ions are created throughout the experiment by electron impact ionization and those created between the DLs are accelerated downstream by only the second DL (and electrons accelerated into the region between the DLs also provide additional ionization ${ }^{12}$ ). The important distinction between the experimental results reported here and the earlier experiments is that here, the multiple ion beams spontaneously appear in a simple divergent magnetic field.

These experimental results are consistent with a number of theoretical predictions. Over forty years ago, one dimensional particle-in-cell (PIC) simulations of ion acoustic shocks in the region of a strong density gradient predicted the formation of DLs at the location of the density gradient and, if the density gradient was steep enough, the formation of wave-like perturbations in density and plasma potential upstream of the shock. ${ }^{13}$ Such a wave-like potential structure could explain these observations. Although more recent PIC simulations have typically yielded only solitary DL structures, ${ }^{14}$ the recent review by Singh ${ }^{15}$ suggests that when perpendicular electric fields develop near density gradients due to differing electron and ion Larmor radii, the perpendicular electric fields are shorted out by conducting boundaries in a laboratory plasma. The resulting parallel electric field may then be localized at a single DL or be spread out across multiple DLs. It is also possible that the electric field structures responsible for these observations are separated radially and that the finite gyroradii of the ions enables different ions to sample different radially localized acceleration regions. Unfortunately, as we are unable to access the last few centimeters of the helicon source before the expansion chamber we are unable to perform LIF measurements of the axial flow at different radial locations near the end of the helicon source. Such measurements are planned in future experiments. However, RFEA measurements of the ion energy distribution function at different radial locations indicate that the 
acceleration regions are quite broad radially and therefore support the interpretation that these measurements are indicative of discrete, axially separated, acceleration regions.

Because the ion beams are relatively cold, it is possible to isolate the beams from the bulk ion population. Maxwellian fits (shown in Figure 5.12a) to each of the three well-defined populations yield identical ion temperatures of $\sim 0.15 \mathrm{eV}$. As noted previously, the large "bulk" ion population is the locally created "downstream" plasma. ${ }^{5}$ In Figure 5.12b, the background ion population in Figure 5.12a has been subtracted from the full measurement to highlight just the portion of the IVDF accelerated by the DL region. After the subtraction, it is clear that there are at least three accelerated ion populations; not just two.

\subsubsection{THEMIS B Observations}

The spontaneous formation of electrostatic structures capable of generating multiple accelerated ion populations in simple expanding magnetic field is a remarkable and new result. The mere possibility that such simple magnetic geometries are capable of producing complex IVDFs has important implications for the interpretation of IVDF measurements in all plasmas. For example, complex IVDFs seen in space are often assumed to be a signature of magnetic reconnection. Shown in Fig 5.14 are two IVDF measurements obtained by the THEMIS spacecraft during a bursty bulk flow event on 26 Feb 2008. Details of the THEMIS spacecraft and the ion distribution measurement process are discussed in Ref. [16,17]. The THEMIS measurements are shown as a function of velocity along the bulk flow direction. At zero velocity, the THEMIS measurements are contaminated with a large background signal due to photoemission and spacecraft charging. Therefore, the low energy portion of the distribution has been removed in Fig. 5.14 and replaced with a dashed line; isolating the ion beams as was done 
with the laboratory measurements. In both THEMIS measurements, two accelerated ion populations are clearly visible. As in Figure 5.12, the accelerated ion populations are superthermal (their flow speeds are much greater than their thermal speeds). Similar complex ion velocity distributions are observed routinely by THEMIS, i.e., the data shown in Figure 5.14 are by no means unique or exceptional.

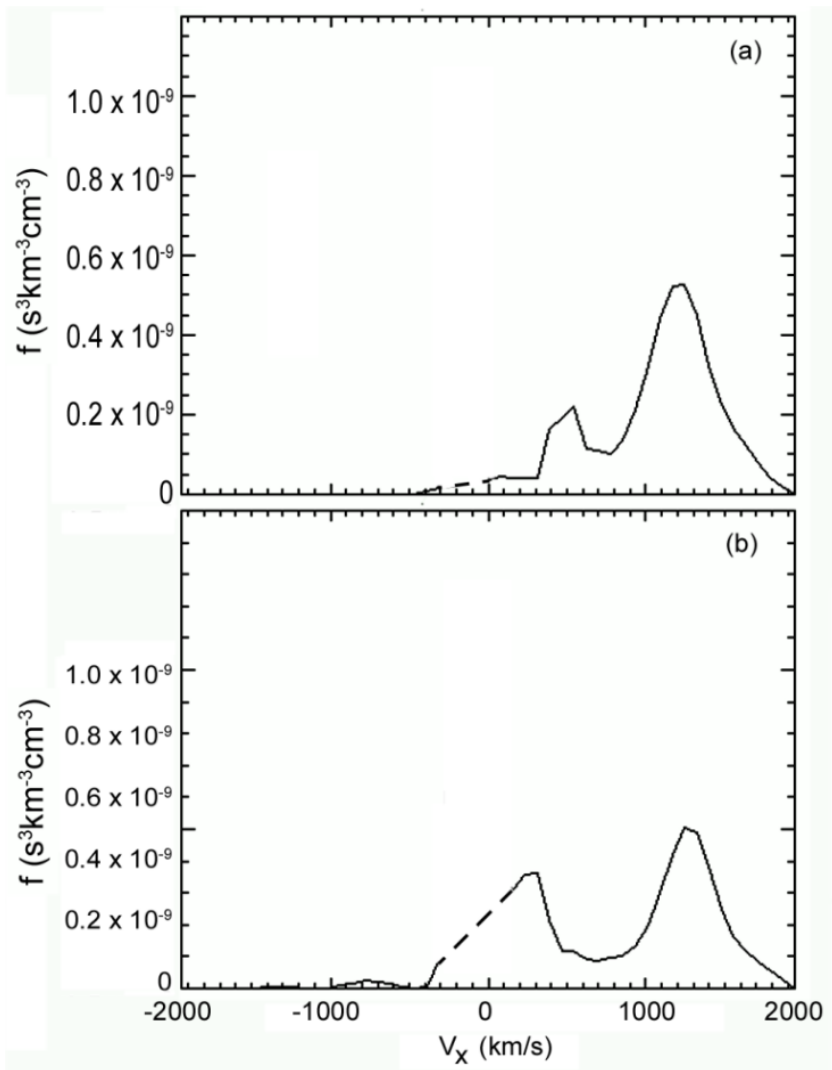

Figure 5.14. The ion velocity distribution function along the outflow direction (reduced to one dimension by integrating over the over two velocity components) for a bursty bulk flow event on 26 Feb 2008 at (a) 11:12:52 and three seconds later at (b) 11:12:55. A large background signal in the measurement at zero velocity due to photoemission and spacecraft charging has been deleted from the figure (the dashed line corresponds to the contaminated region of the distribution). Two accelerated ion populations appear in both measurements. 


\subsubsection{Reconnection Simulations}

As further evidence that complex IVDFs arising during magnetic reconnection are evidence of discrete electric field regions, an IVDF from an implicit two-and-a-half dimensional PIC simulation of magnetic reconnection obtained from the University of Colorado-Boulder is shown in Figure 5.16. The simulation includes a guide field (equal in strength to the reconnecting field) and the computational domain size is $L_{x} \times L_{y}=40 d_{i} \times 20 d_{i}$ (where $d_{i}=c / \omega_{p i}$ is

the ion inertial length, $c$ is the speed of light, $\omega_{p i}=\sqrt{4 \pi n e^{2} / m_{i}}$ in cgs units is the ion plasma frequency, and $e$ is the electron charge). Periodic boundary conditions are assumed in the $x$ direction and perfect electric conductor boundaries are set at $y=0$ and $y=L_{y}$. The reconnection simulation starts with a classic Harris sheet ${ }^{18}$ of high density particles surrounded by background particles with a density an order of magnitude lower and a magnetic field profile given by a hyperbolic tangent function. Additional details about the simulation and physics assumptions inherent in the model are discussed in Ref. [19]. The $x$ direction is along the bulk outflow direction and the distribution has been integrated over the direction perpendicular to the bulk flow. The velocities are normalized to the reference Alfvén speed $v_{A}=B_{o} /\left(\mu_{o} n_{i o} m_{i}\right)^{1 / 2}$, where $B_{o}$ is the background magnetic field strength, $m_{i}$ is the ion mass, and $n_{i o}$ is the initial maximum Harris-sheet ion density. The simulation distribution function is sampled at $20 d_{i}$ downstream of the reconnection site along the outflow axis many ion cyclotron periods after the onset of reconnection. As in the laboratory and space measurements, multiple accelerated ion populations appear in the IVDF (as well as a background ion population). The total ion distribution at this location includes one population crossing the separatrix without sampling the Hall electric field near the X-line and other populations that have been accelerated by discrete 
Hall electric field structures that appear near the X-line and along the separatrices. ${ }^{20}$ Further $^{2}$ downstream, the populations merge into a single, broad, "hot”, IVDF.

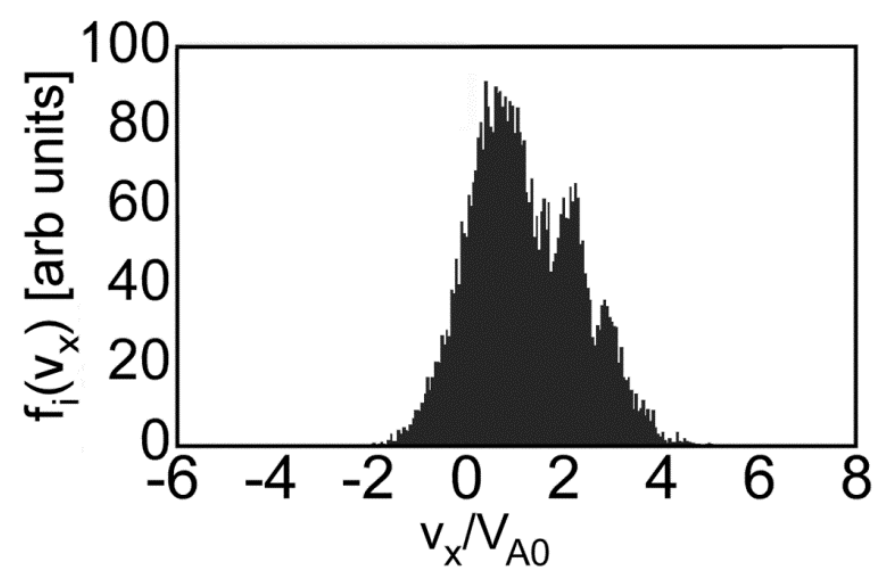

Figure 5.16. Ion velocity distribution (as a function of velocity normalized to the initial Alfvén velocity) along the outflow direction from a particle-in-cell numerical simulation of magnetic reconnection. The distribution is obtained 20 ion inertial lengths downstream of the reconnection site and is integrated over the other two coordinate directions. The ion velocity distribution includes two accelerated ion populations plus a stationary background population.

\subsubsection{Implications of Multi-Beam Results for Reconnection Driven Ion Heating Studies}

Clearly, the appearance of a complex IVDFs can be a signature of magnetic reconnection. However, based on these new laboratory observations, we conclude that the mere existence of a complex IVDF is not sufficient to posit the existence of magnetic reconnection. Such IVDFs could simply result from plasma expansion and production in a divergent magnetic field (or flows of different source plasmas into a common divergent field region). 
Consider the rapid and intense ion heating typically associated with magnetic reconnection in laboratory experiments. ${ }^{21}$ The heating occurs more rapidly than any collisional or viscous timescale in the plasma and the "heated" ion population is well described by a high temperature Maxwellian distribution plus a power-law high-energy tail. ${ }^{22}$ How the ions are heated so rapidly remains an open question in reconnection dominated experiments. However, nearly all such heating measurements are fundamentally unable to resolve structure within the IVDF at the spatial scale of a reconnection layer. Either the spatial resolution of the diagnostic technique exceeded the reconnection scale, the technique employed line integrated measurements, or the velocity resolution of the technique was insufficient to differentiate between complex structure and a broad, hot, velocity distribution. ${ }^{23,24}$ Even diagnostic methods with spatial resolution comparable to reconnection layer scale still average over large spatial regions because of the large ion gyroradius of the heavy impurity species used for the measurement. ${ }^{25}$ Therefore, although broad IVDFs are usually interpreted to be indicative of ion heating, typical observations are equally consistent with unresolved IVDFs containing multiple accelerated ion populations. ${ }^{26,27}$

As an example of how the presence of ion beams could be misinterpreted as ion heating, consider that a large fraction of the ion temperatures reported by space-based instruments are based on a straightforward and automatic calculation of the 2nd moment of the entire measured IVDF. Although the LIF-measured temperature of each ion beam population in Fig. 5.12a is 0.16 $\mathrm{eV}$, the average kinetic energy in the frame of the flow, i.e., the 2nd moment of the IVDF, yields an ion temperature of well over $1 \mathrm{eV}$. Thus, similar naïve analysis of the IVDF shown in Figure 5.12a would conclude that the downstream ion temperature was an order of magnitude hotter than the upstream temperature, even though no significant heating of the ions actually occurred. 
It is only because THEMIS, with its very high time resolution ion instrument, is capable of resolving relatively small spatial scales that the ion beams are resolvable in the data of Fig. 5.14.

If we assume that ion dynamics during phenomena such as magnetic reconnection are not dominated by thermal processes or wave-particle interactions, but instead result from reversible acceleration in discrete electric fields (as has been shown to occur for single test particles in simulations $^{28}$ ) of varying magnitude and orientation, the rate of ion energization (heating) should depend solely on the energy gained by ions falling through such electric fields:

$$
\frac{\Delta\left(\frac{m v^{2}}{2}\right)}{\Delta \mathrm{t}} \approx \sqrt{d q^{3} E^{3} / 2 m}
$$

where $E$ is the electric field in the DL of thickness $d$ and the heating rate is determined by the transit time of the ions in the DL. In other words, the energization rate of ions of different charge-to-mass $(q / m)$ ratios should scale as $\left(q^{3} / m\right)^{1 / 2}$ and should be independent of the magnitude of the magnetic fluctuation amplitude. In the Madison Symmetric Torus (MST), the ion temperature during magnetic reconnection doubles or triples in less than $10 \mu \mathrm{s}$. Heating models based on cyclotron heating from magnetic fluctuations and viscous damping of reconnection flows have been proposed. Yet, two decades of measurements have failed to find any significant correlation between ion heating levels and the levels of magnetic fluctuations (levels of ion heating have been shown to correlate with the overall change in stored magnetic energy during magnetic reconnection ${ }^{29}$ ). Recently, the MST experiment reported that their first studies of ion heating for impurity ions of different charge-to-mass ratios demonstrated that the parallel ion heating rate depends on the $(q / m)$ ratio during magnetic reconnection. ${ }^{30}$ However, the overall $(q / m)$ ratio range was too limited to distinguish between $\left(q^{3} / m\right)^{1 / 2}$ or linear $(q /$ 
$m$ ) scaling. We hypothesize that ions passing through a multitude of tightly packed and randomly oriented reconnection sites with a distribution of total energization "strengths" might rapidly acquire a distribution of three-dimensional velocities that appear to result from a single hot near-Maxwellian parent distribution. Fig. 5.16a shows the latest scaling model proposed by the MST group to explain ion heating rates. Increase in temperature is plotted against the atomic number of the particle divided by its mass $(\mathrm{z} / \mathrm{m})$. Figure $5.16 \mathrm{~b}$ shows a model of scaling predicted by acceleration in a randomly oriented electric field. This series of reversible accelerations leads to a continuous increase in temperature (heating) with no appreciable way discern its origin. These two plots demonstrate the equivocal nature of the MST model.
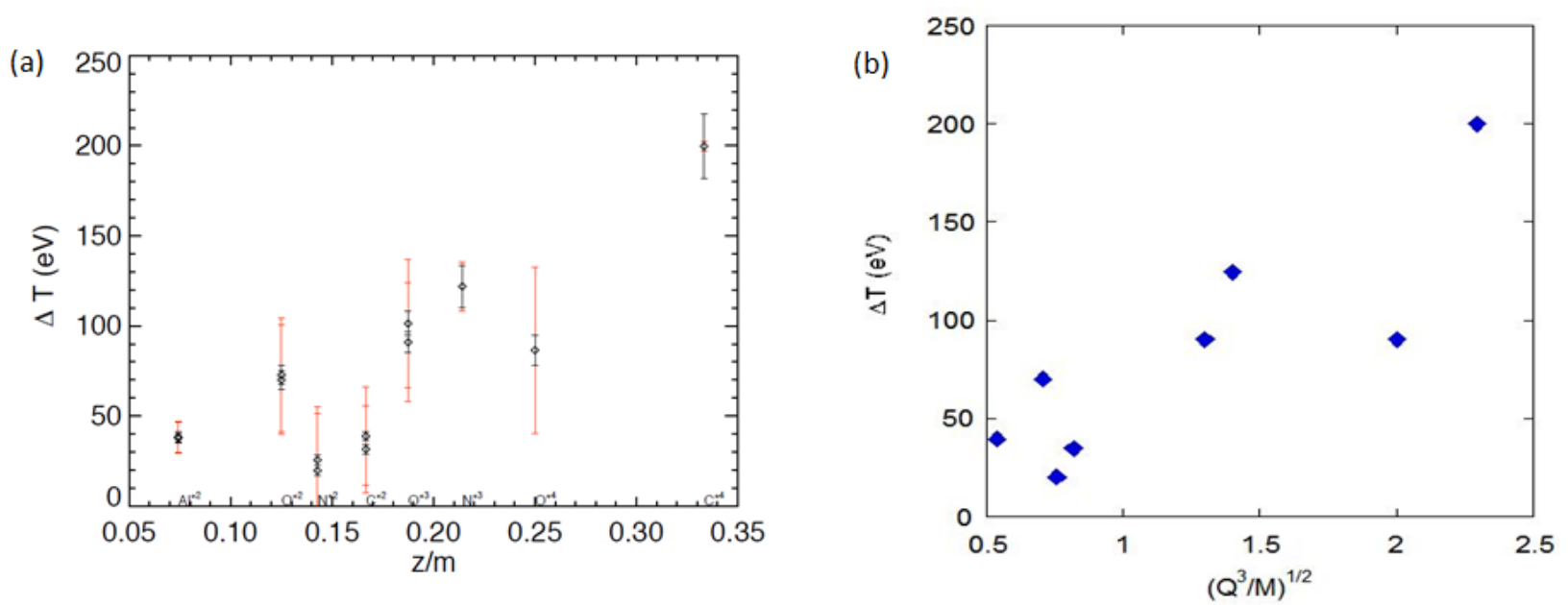

Figure 5.16. (a) Santosh Kumar et al. (submitted); MST ion heating data plotted against $z / m$ (b) Scaling predicted by acceleration in randomly oriented electric fields model. 


\subsection{Comparison of RFEA and LIF Data}

Although LIF has provided some remarkable new results presented in this work thus far, it is not without limitations. The RFEA provides another measurement technique to interrogate ion populations in places where LIF cannot function due to metastable quenching. RFEAs can also provide plasma potential measurements

Fig 5.17 shows several axial LIF measurements performed in the LEIA expansion chamber. As the axial position $z$ increases (the scan location moves further downstream of the DL), the signal of the beam decreases. At the $z=79 \mathrm{~cm}$, very little evidence of the beam remains. Two possible reasons for this are (1) the beam is no longer present and (2) a beam is present but there are no longer enough metastable ion states to generate LIF signal. The latter has shown to be true in other devices, but LEIA is much larger than those experiments, with more chamber length to interrogate. The RFEA probe was thus used at the following source parameters to see if it had the ability to detect a beam where LIF could not: 900 G in the HELIX, $0 \mathrm{G}$ in LEIA, rf frequency of 9.5 MHz, rf power of $650 \mathrm{~W}$, flow of 3.0 SCCM, HELIX Pressure of 0.7 mTorr, and $9.9 \times 10^{-5}$ Torr in LEIA. 


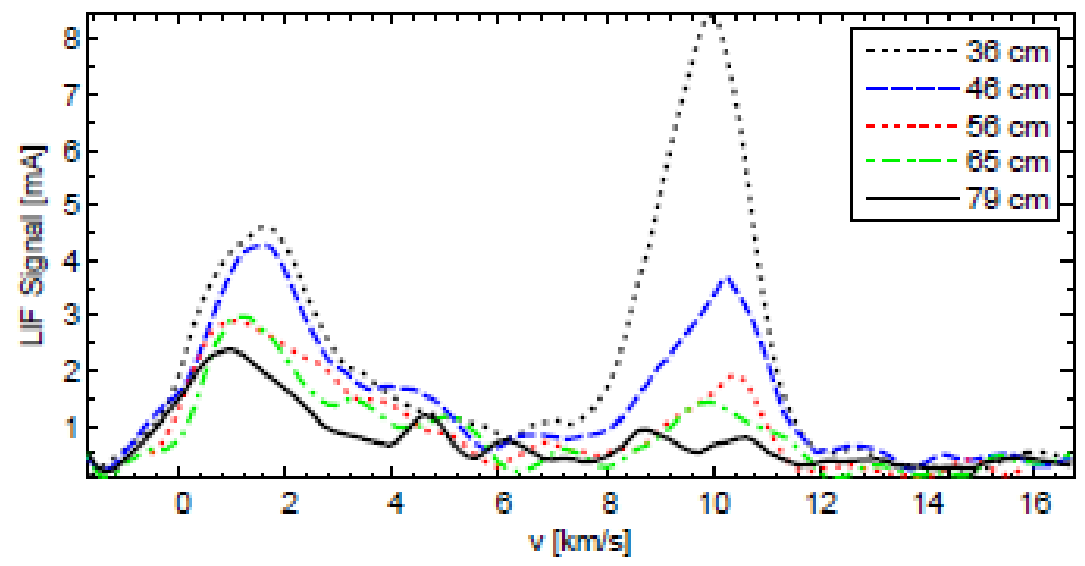

Figure 5.17. LIF measurements at $900 \mathrm{G}$ in the source, $0 \mathrm{G}$ in the expansion chamber, $\mathrm{rf}$ frequency of $9.5 \mathrm{MHz}$, $\mathrm{rf}$ power of $650 \mathrm{~W}$, flow of 3.0 SCCM, Pressure in source $0.7 \mathrm{mTorr}$, and $9.9 \times 10^{-5}$ Torr in the expansion chamber. The beam, when detected remains at $10 \mathrm{~km} / \mathrm{s}$.

Figure 5.18a shows an actual RFEA measurement in the center of LEIA, $80 \mathrm{~cm}$ downstream. The black curve comes from the configuration shown in Fig. 3.10b, where the plasma sees the repeller before the discriminator. The red curve was obtained by placing the discriminator first (Fig. 3.10c). Shown in Fig. 5.18b are the derivatives of the RFEA signal versus discriminator voltage. As noted earlier, in the case of an undrafted, pure Maxwellian velocity distribution, the derivative yields the ion energy distribution. The background and beam populations in both figures 5.17 and $5.18 \mathrm{~b}$ show a similar spread in energies for both populations. The beam population amplitude decreases with increasing downstream distance in the LIF measurements but the energy remains unchanged, consistent with previous observations attributed to metastable quenching. The RFEA beam signal at $80 \mathrm{~cm}$ downstream is much larger than in the LIF measurements, again consistent with the beam continuing on downstream but becoming invisible to LIF interrogation because of depopulation of the initial metastable state needed for LIF. 


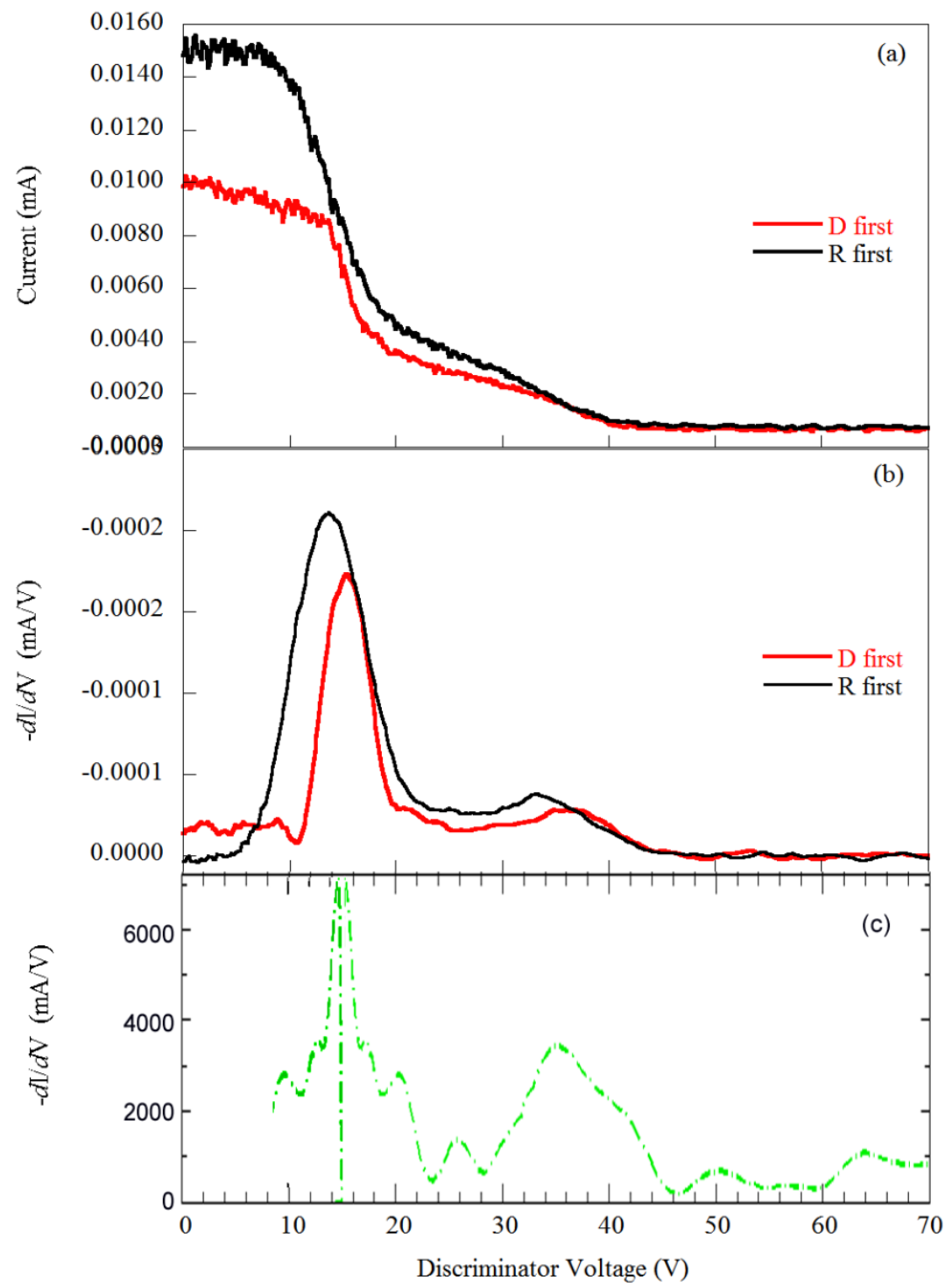

Figure 5.18. RFEA measurements obtained at $z=80 \mathrm{~cm}$. (a) The $\mathrm{I}-\mathrm{V}$ trace and (b) the corresponding derivative as a function of voltage. Figure courtesy of the UiT group. The black curve is for the RFEA configuration with the repeller is placed ahead of the discriminator and the red curve is when the discriminator is placed first. (c) the LIF signal for $\mathrm{z}=40 \mathrm{~cm}$ in Fig. 5.17, re-plotted as an ideal RFEA for comparison purposes.

Note two features from the grid placement comparison: (1) The repeller-first configuration has wider populations in terms of voltage (analogous to energy) and (2) the populations in the repeller-configuration are shifted downward in energy when compared to the 
discriminator-first configuration. Both observations indicate additional lower energy ions were collected by the repeller-first probe. ${ }^{31,32}$ The repeller-first geometry lacks the ability to focus the ions as well as the other configuration, presumably because it is creating a larger sheath, letting more low energy ions into the probe.

Fig. 5.19 shows how the same IVDFs in Fig 5.17 would appear if measured with an ideal RFEA, assuming a plasma potential voltage of $15 \mathrm{~V}$ and by assuming

$$
V=\frac{m_{i} v^{2}}{2 e}+V_{p}
$$

where $V$ is the ideal RFEA voltage, $v$ is the velocity of the ion, $m_{i}$ is the mass of the ion, $e$ is the fundamental unit of electric charge and $V_{p}=15 \mathrm{~V}$ is the floating potential of the plasma. The I-V trace that the ideal RFEA would measure for each IVDF is shown in Fig. 5.17a. Note that the artificially generated IVDF I-V curves are cut off for velocities less than then plasma potential as there is no way for ions moving away from an ideal RFEA to enter the probe. Shown in Fig. 5.18c is the $z=40 \mathrm{~cm}$ LIF data from Figure 5.19 again re-plotted as an ideal RFEA measurement. The background population in Fig. 5.18c is reflected across the $V=15 \mathrm{~V}$ axis to allow for to better be able to compare the widths in energy space generated by the two different techniques. The LIF measurement clearly yields an ion energy distribution much narrower, colder, than the RFEA measurements. The artificially "hot" RFEA measurements have been noted before in previous studies. ${ }^{33}$ 


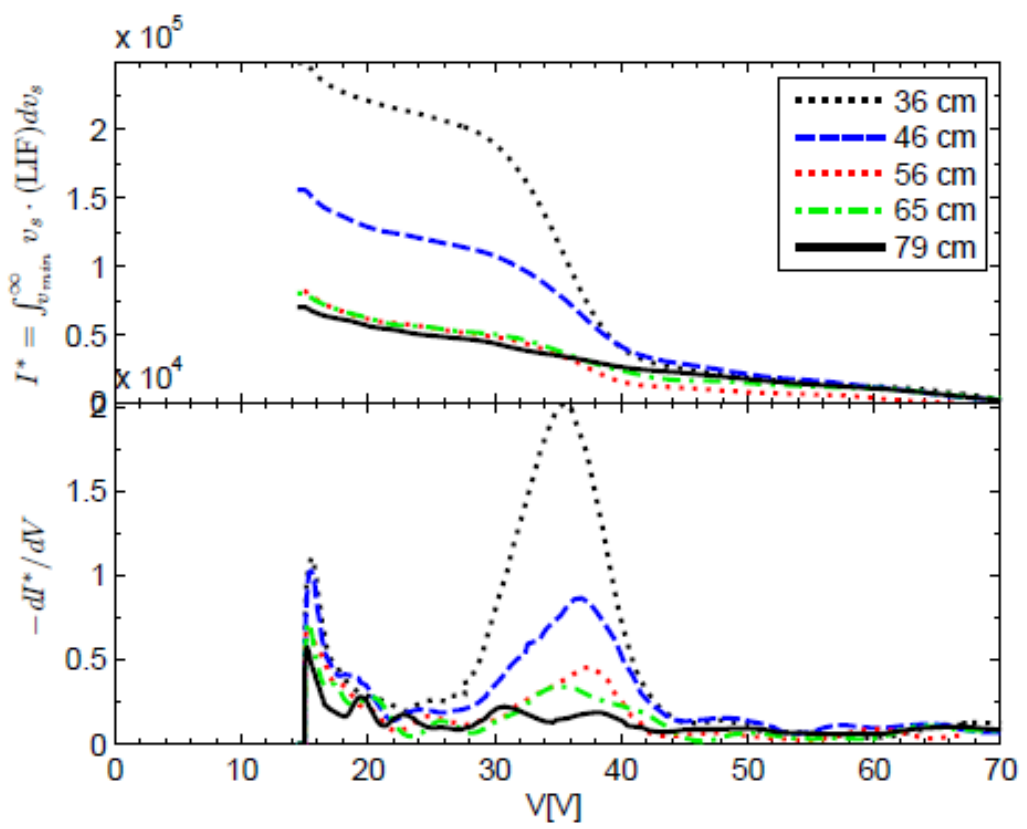

Figure 5.19. LIF data presented in the form of ideal RFEA measurements. (a) The I-V trace and (b) the corresponding derivative as a function of voltage. Figure courtesy of the UiT group.

Comparing background temperatures quantitatively, the repeller-first RFEA measurement gave an ion temperature of $6.5 \mathrm{eV}$ while the discriminator-first system gave an ion temperature $5.1 \mathrm{eV}$. The LIF temperature measurements were $0.5 \mathrm{eV}$ at $79 \mathrm{~cm}$ and $0.7 \mathrm{eV}$ at $36 \mathrm{~cm}$ on the $\mathrm{z}$ axis. 


\section{Chapter 5 References}

${ }^{1}$ E. E. Scime, I. A. Biloiu, J. Carr, Jr., S. Chakraborty Thakur, M. Galante, A. Hansen, S. Houshmandyar, A. M. Keesee, D. McCarren, S. Sears, C. Biloiu, and X. Sun, Phys. Plasmas 17, 055701 (2010).

${ }^{2}$ I. A. Biloiu and E. E. Scime, Appl. Phys. Lett. 95, 051504 (2009).

${ }^{3}$ N. Singh, Phys. Plasmas 18, 122105 (2011).

${ }^{4}$ J. Carr, Jr., P. A. Cassak, M. Galante, A. M. Keesee, G. Lusk, R. M. Magee, D. McCarren, E. E. Scime, S. Sears, R. Vandervort, N. Gulbrandsen, M. Goldman, D. Newman, and J. P. Eastwood, Phys. Plasmas, 20, 072118 (2013).

${ }^{5}$ X. Sun, A. M. Keesee, C. Biloiu, E. E. Scime, A. Meige, C. Charles C and R. Boswell Phys. Rev. Lett. 95, 025004 (2005).

${ }^{6}$ J. Birn, A.V. Artemyev, D.N. Baker, M. Echim, M. Hoshino, L.M. Zelenyi , Space Sci. Rev. 173 49-102 (2012).

${ }^{7}$ B.A. Bryant, R. Bingham, U. Deangelis, Phys. Rev. Lett. 68, 37 (1992).

${ }^{8}$ S. A. Cohen, N.S. Siefert, S. Stange, R.F. Boivin, E.E. Scime, and F.M. Levinton, Phys. Plasmas 10, 2593 (2003).

${ }^{9}$ F. Skiff, G. Bachet, and F. Doveil, Phys. Plasmas 8, 3139 (2001).

${ }^{10}$ N. Gulbrandsen, W. J. Miloch, and A. Fredriksen, Contrib. Plasma Phys. 53, 27 (2013).

${ }^{11}$ X. Sun, S. Cohen, and E. E. Scime, Phys. Plasmas 12, 103509 (2005).

${ }^{12}$ S. Chakraborty Thakur, Z. Harvey, I. A. Biloiu, A. Hansen, R. A. Hardin, W. S. Przybysz, and E. E. Scime, Phys. Rev. Lett. 102, 035004 (2009).

${ }^{13}$ R.J. Mason, Phys. Fluids 14, 1943 (1971). 
${ }^{14}$ A. Meige, R. W. Boswell, C. Charles, and M. M. Turner, Phys. Plasmas 12, 052317 (2005).

${ }^{15}$ N. Singh, Phys. Plasmas 18, 1 (2011).

${ }^{16}$ J.P. McFadden, C.W. Carlson, D. Larson, M. Ludlam, R. Abiad, B. Elliott, P. Turin, M. Marckwordt, and V. Angelopoulos, Space Sci. Rev. 141, 277 (2008).

${ }^{17}$ A. Meige, R. W. Boswell, C. Charles, and M. M. Turner, Phys. Plasmas 12, 052317 (2005).

${ }^{18}$ E.G. Harris, Nuovo Cimento 23, 115 (1962).

${ }^{19}$ A. Divin, G. Lapenta, S. Markidis, D. L. Newman, and M. V. Goldman, Phys. Plasmas 19, 042110 (2012).

${ }^{20}$ M. A. Shay, J. F. Drake, R. E. Denton, D. Biskamp, J. Geophys. Res. 103, 9165 (1998).

${ }^{21}$ E. Scime, S. Hokin, N. Mattor, C. Watts, Phys. Rev. Lett. 68, 2165 (1992).

${ }^{22}$ R. M. Magee, D.J. Den Hartog, S.T. A. Kumar, A.F. Almagri, B.E. Chapman, G. Fiksel, V.V. Mirnov, E.D. Mezonlin, J.B. Titus, Phys. Rev. Lett. 107, 065005 (2011).

${ }^{23}$ A.K. Hansen, Matthew Galante, Dustin McCarren, Stephanie Sears, and E.E. Scime, Rev. Sci. Instrum. 81, 10D701 (2010).

${ }^{24}$ V.H. Chaplin, M. Brown, D.H. Cohen, T. Gray, C.D. Cothran, Phys. Plasmas 16, 042505 (2009).

${ }^{25}$ M.R. Brown, C.D. Cothran, T. Gray, C.E. Myers, E.V. Belova, Phys. Plasmas 19, 080704 (2012).

${ }^{26}$ T. Intrator, J. Menard, N. Hershkowitz, Phys. Fluids B 5, 806 (1993).

${ }^{27}$ R. L. Merlino and J.J. Loomis, Phys. Fluids B 2, 2865 (1990).

${ }^{28}$ J.F. Drake, M. Swisdak, T.D. Phan, P.A. Cassak, M.A. Shay, S.T. Lepri, R.P. Lin, E. Quataert, T.H. Zurbuchen, J. Geophys. Res. 114, A05111 (2009). 
${ }^{29}$ S. Gangadhara, D. Craig, D.A. Ennis, D.J. Den Hartog, G. Fiksel, S.C. Prager, Phys. Plasmas 15, 056121 (2008).

${ }^{30}$ D.J. Den Hartog, Ion energization during magnetic reconnection in the RFP laboratory plasma, Bull Am. Phys. Soc. 57, BI2.00002 (2012).

${ }^{31}$ V. Kanarov, D. Siegfried, P. Sferlazzo, A. Hayes, and R. Yevtukhov, Rev. Sci. Instrum., 79, 093304 (2008).

${ }^{32}$ N. Gulbrandsen, W. J. Miloch, and Å. Fredriksen, Contrib. Plasma Phys. 53, No. 1, 27 (2013).

${ }^{33}$ Z. Harvey, S. Chakraborty Thakur, A. Hansen, R. A. Hardin, W. S. Przybysz and E. E. Scime, Rev. Sci. Instrum. 79, 10F314 (2008). 


\section{Chapter 6: Summary}

The time resolved LIF DL studies described in this work are consistent with the hypothesis that the creation of strong DLs in expanding plasmas for plasma propulsion or other applications may be self-limited through instability growth. These results suggest that a similar mechanism might play a role in the collapse of naturally occurring DLs. The two cases examined employed pulsed plasma whose source parameters were selected based on steady-state DL stability experiments by Chakraborty Thakur et al. ${ }^{1}$ The plasma conditions for both pulsed plasma cases were identical except that one used a high magnetic mirror ratio and the other had a moderate magnetic mirror ratio. At the moderate magnetic mirror ratio, a DL was observed in steady state plasmas but there was no evidence of an instability. In pulsed plasmas, time resolved LIF measurements revealed the presence of a DL in both cases at the start of the discharge. However, in the higher magnetic mirror ratio case, the DL collapsed once the electrostatic fluctuations appeared.

In higher time resolution studies, in which the LIF signal was acquired with a much larger frequency bandwidth, a clear correlation between the instability and the ion beam resulting from the DL was measured. Only the ion beam portion of the ion velocity distribution was correlated with the instability amplitude. The background population demonstrated no statistically significant correlation with the instability. These correlation measurements provide the first statistical evidence that laboratory CFDLs are linked to self-limiting instability growth. A continuous wavelet analysis of the fluctuation measurements provided clear evidence for the appearance, increase in amplitude, and then disappearance of the instability several times in a single plasma pulse. 
During the DL stability studies, surprising measurements of the spontaneous creation of multiple beams in a simple divergent magnetic field in a laboratory were obtained. The structure of the measured ion velocity distributions is remarkably similar to ion velocity distributions observed in regions of magnetic reconnection in space and in numerical simulations. These observations suggest there is no significant difference in IVDFs arising from reconnection or a simple divergent magnetic field. It is only because the space observations, such as the THEMIS observations, include magnetic field measurements, that it is possible to identify the THEMIS event as a bursty bulk flow associated with magnetic reconnection. The similarity between the ion velocity distributions measured in these experiments and those observed in simulations and in space has other far-reaching implications:

(1) Ion heating commonly associated with magnetic reconnection may be more accurately described as the superposition of multiple ion populations accelerated by a collection of electrostatic fields.

(2) The complex structures in ivdf measurements seen in space and simulation, i.e., multiple beams of varying relative magnitudes, do not necessarily require impulsive phenomena, such as magnetic reconnection, for creation.

(3) If the ion dynamics during magnetic reconnection are also dominated by acceleration in electric fields of varying magnitude and orientation, the rate of ion energization (heating) during magnetic reconnection should depend solely on the energy gained by ions falling through such electric fields.

Through a collaboration between UiT and WVU, a new RFEA probe was benchmarked on the low pressure CFDL plasmas produced in WVU HELIX-LEIA. LIF was used to confirm 
the RFEAs ability to detect a beam when one was present. The RFEA was also able to detect the presence of a beam when LIF techniques were limited by metastable quenching. A series of experimental tests described in this work provided a clear sense as to which RFEA configuration best handles the issue of ion focusing. Placing the discriminator in front of the repeller, so that low energy ions were reflected immediately after the probe entrance, reduced the artificial broadening of the measured IED due to ion focusing effects.

A natural extension to this work will be to investigate multi-beam parameters with time resolved data acquisition techniques. High time resolution LIF will require identification of the laser frequencies at which the three populations (beam, secondary beam, and background) signal are maximum and then recording of the fluctuation and time series data at those particular frequencies. Wavelet and Fourier analysis should be performed on the signals in same manner it was presented in Chapter 4, but an additional analysis methodology, the cross-wavelet power spectrum, should be considered. For two time series $X$ and $Y$, the cross-wavelet spectrum is defined $\mathrm{by}^{2}$

$$
W_{n}^{X Y}(s)=W_{n}^{X}(s) W_{n}^{Y *}(s)
$$

where $W_{n}^{X}$ is the wavelet transform of $X$ and $W_{n}^{Y *}$ is the complex conjugate of the wavelet transform of $Y$. The cross-wavelet spectrum is complex therefore it may be more beneficial to record the cross-wavelet power, $\left|W_{n}^{X Y}(s)\right|$. The cross-wavelet power spectrum provides a continuous measurement of correlation between two signals, and will provide a measure of the time dependent correlation between ion beam components and electrostatic fluctuations throughout the plasma pulse. 
A missing piece of information in all these experiments is the electron energy distribution function (EEDF). This difficult measurement is possible with a carefully rf compensated Langmuir probe. The EEDF is determined from a plot of the second derivative of the I-V trace versus the applied voltage. ${ }^{3}$ Such a measurement would provide additional details of the DL formation mechanism and would enable additional comparisons with results from the ANU group. ${ }^{4}$ Another important additional measurement that should be pursued is to map out the potential structure throughout the expansion region. ${ }^{5}$ Such a measurement presents significant engineering challenges, but it could be accomplished with a doglegged RFEA probe similar to the one used in CHI-KUNG. 


\section{Chapter 6 References}

${ }^{1}$ S. Chakraborty Thakur, Z. Harvey, I. A. Biloiu, A. Hansen, R. A. Hardin, W. S. Przybysz, and E. E. Scime, Phys. Rev. Lett., 102, 035004 (2009).

${ }^{2}$ C. Torrence and G. P. Compo, Bull. Amer. Meteor. Soc., 79, 61 (1998).

${ }^{3}$ K. Takahashia, C. Charles, R. W. Boswell, T. Kaneko, and R. Hatakeyama, Phys. Plasmas 14, 114503 (2007).

${ }^{4}$ K. Takahashi, C. Charles, R. W. Boswell, and T. Fujiwara, Phys. Rev. Lett., 107, 035002 (2011).

${ }^{5}$ C. Charles, R. W. Boswell, R. Hawkins, Phys. Rev. Lett., 103, 095001 (2009). 